\title{
A high-resolution spectroscopic survey of late-type stars: chromospheric activity, rotation, kinematics, and age ${ }^{\star, \star \star}$
}

\author{
J. López-Santiago ${ }^{1}$, D. Montes ${ }^{1}$, M. C. Gálvez-Ortiz ${ }^{2}$, I. Crespo-Chacón ${ }^{1}$, R. M. Martínez-Arnáiz ${ }^{1}$, \\ M. J. Fernández-Figueroa ${ }^{1}$, E. de Castro $^{1}$, and M. Cornide ${ }^{1}$
}

\author{
1 Departamento de Astrofísica y Ciencias de la Atmósfera, Universidad Complutense de Madrid, 28040 Madrid, Spain \\ e-mail: jls@astrax.fis.ucm.es \\ 2 Centre For Astrophysics Research, University of Hertfordshire, College Lane, Hatfield, Hertfordshire, AL10 9AB, UK
}

Received 9 October 2009 / Accepted 5 February 2010

ABSTRACT

\begin{abstract}
Aims. We present a compilation of spectroscopic data from a survey of 144 chromospherically active young stars in the solar neighborhood, which may be used to investigate different aspects of its formation and evolution in terms of kinematics and stellar formation history. The data have already been used by us in several studies. With this paper, we make all these data accessible to the scientific community for future studies on different topics.

Methods. We performed spectroscopic observations with echelle spectrographs to cover the entirety of the optical spectral range simultaneously. Standard data reduction was performed with the IRAF ECHELLE package. We applied the spectral subtraction technique to reveal chromospheric emission in the stars of the sample. The equivalent width of chromospheric emission lines was measured in the subtracted spectra and then converted to fluxes using equivalent width-flux relationships. Radial and rotational velocities were determined by the cross-correlation technique. Kinematics, equivalent widths of the lithium line $\lambda 6707.8 \AA$ and spectral types were also determined.

Results. A catalog of spectroscopic data is compiled: radial and rotational velocities, space motion, equivalent widths of optical chromospheric activity indicators from Ca II H \& K to the calcium infrared triplet and the lithium line in $\lambda 6708 \AA$. Fluxes in the chromospheric emission lines and $R_{\mathrm{HK}}^{\prime}$ are also determined for each observation of a star in the sample. We used these data to investigate the emission levels of our stars. The study of the $\mathrm{H} \alpha$ emission line revealed two different populations of chromospheric emitters in the sample, clearly separated in the $\log F_{\mathrm{H} \alpha} / F_{\mathrm{bol}}-(V-J)$ diagram. The dichotomy may be associated with the age of the stars.
\end{abstract}

Key words. Galaxy: stellar content - solar neighborhood - stars: late-type - stars: activity - stars: chromospheres

* Based on observations made with the $2.2 \mathrm{~m}$ telescope of the German-Spanish Astronomical Centre, Calar Alto (Almería, Spain), operated jointly by the Max-Planck-Institute for Astronomy, Heidelberg, and the Spanish National Commission for Astronomy; the Nordic Optical Telescope (NOT), operated on the island of La Palma jointly by Denmark, Finland, Iceland, Norway and Sweden, in the Spanish Observatorio del Roque de Los Muchachos of the Instituto de Astrofísica de Canarias; the Isaac Newton Telescope (INT) operated on the island of La Palma by the Isaac Newton Group in the Spanish Observatorio del Roque de Los Muchachos of the Instituto de Astrofísica de Canarias; with the Italian Telescopio Nazionale Galileo (TNG) operated on the island of La Palma by the Centro Galileo Galilei of the INAF (Istituto Nazionale di Astrofisica) at the Spanish Observatorio del Roque de Los Muchachos of the Instituto de Astrofísica de Canarias; and with the Hobby-Eberly Telescope (HET) operated by McDonald Observatory on behalf of The University of Texas at Austin, the Pennsylvania State University, Stanford University, Ludwig-Maximilians-Universität München, and Georg-August-Universität Göttingen. This research has made use of the SIMBAD database and VizieR catalog access tool, operated at CDS, Strasbourg, France.

$\star \star$ Tables A.1-A.4 and reduced spectra are also available in electronic form at the CDS via anonymous ftp to cdsarc.u-strasbg. fr (130.79.128.5) or via

http://cdsweb.u-strasbg.fr/cgi-bin/qcat?J/A+A/514/A97

\section{Introduction}

The velocity (or phase) space in the solar neighborhood is rather complicated. In particular, the $U V$-plane shows different structures that are associated with the Galactic potential characteristics. At large scales, the $U V$-plane is dominated by long branches (Skuljan et al. 1999) related to dynamical perturbations altering the kinematics of the solar neighborhood (Famaey et al. 2005), including the resonance of the rotating bar (Famaey et al. 2007; Antoja et al. 2009). The fine structure of the velocity distribution of disk stars is more likely related to the existence of the classic Eggen moving groups (see Montes et al. 2001a, for a review of the young moving groups problem). In fact, the substructures found inside the long branches appear to have on average different ages (Asiain et al. 1999; Antoja et al. 2008), which agree with the idea of the moving groups being formed by coeval stars. These young substructures are mixed in phase space with old stars and it is difficult to discern between young and old stars only by their kinematics. In some cases, the vertical component of the Galactic velocity $(W)$ can be used to reject membership of the star in one of the young moving groups. The vertical velocity dispersion in the solar vicinity is only dependent on the scale height (Toth \& Ostriker 1992). Old stars (2-10 Gyr) present higher scale heights than young stars $(<1 \mathrm{Gyr})$, and hence high 
values of $W$ are more typical of old stars. However, the division between high and low velocity is subtle.

From the classical point of view of the Galactic velocity ellipsoid, early-type stars show lower dispersion in their Galactic velocities $(U, V$, and $W)$ than late-type stars and are usually restricted to the spiral arms or star-forming regions. This result has already been interpreted in the past as a consequence of the increase of dispersion with increasing age (see Mihalas \& Binney 1981). A recent study of the kinematics of $M$ dwarfs in the solar vicinity by Bochanski et al. (2005) shows that the most chromospherically active (i.e. youngest) stars really show lower velocity dispersion than non-active (i.e. older) stars. Because of this and their ubiquity, late-type stars are excellent tracers of the Galactic potential (Bochanski et al. 2007). Distinguishing between young and old stars is then crucial to an understanding of the kinematics of the Galaxy and of stellar formation history in our neighborhood (López-Santiago et al. 2007).

An effort has been made in the past to quantify the proportion of young and old stars in samples of candidates of the young moving groups and associations using different age indicators. In particular, the level of magnetic activity is a powerful tool for this purpose (see López-Santiago et al. 2009). The activity level of late-type stars is inversely correlated with age due to the decrease of stellar rotation with increasing age (e.g. Skumanich 1972; Noyes et al. 1984; Rutten \& Schrijver 1987; Randich et al. 1996; Pizzolato et al. 2003). The rotation-age-activity relationship persists into the fully convective regime (e.g. Reiners \& Basri 2007; West et al. 2008). M dwarfs indeed have finite active lifetimes (West et al. 2009). Therefore, the level of magnetic activity is a good indicator of age for late-F to M dwarfs.

For four years, between 1999 and 2002, our group carried out a spectroscopic survey of chromospherically active latetype stars in the solar neighborhood, selected from a list of possible members of classical young stellar kinematic groups (Montes et al. 2001a). The aim was to use different age indicators (chromospheric activity, lithium, rotational velocity) to constrain some properties of the moving groups and to study in detail the existence of age subgroups in large samples of stars selected, mainly, by their kinematics. Since then, the information derived from this project has been extensively used by us in different works. For instance, Montes et al. (2001b) carried out a multiwavelength study of a sample of active stars, which allowed us to constrain the age of 14 young late-type stars. The results put constraints on the age of some young moving groups. Also, López-Santiago et al. (2003) studied the relation between variations observed in the photosphere and chromosphere of PW And using data from this spectroscopic survey.

More recently, part of the data from this survey was used to investigate nearby young moving groups (López-Santiago et al. 2006). A consequence of this study was the confirmation of the existence of two age subgroups in the previously discovered AB Dor moving group (Zuckerman et al. 2004). Through age indicators (mainly chromospheric activity and lithium abundance), we showed that the Local Association is indeed a mixture of subgroups of stars with different ages. In López-Santiago et al. (2009), we used the information on the chromospheric activity of the stars provided by the spectroscopic survey, together with X-ray data from the ROSAT All Sky Survey (RASS) to quantify the contamination by old main-sequence stars of the sample of possible members of the Local Association in Montes et al. (2001a).

At present, two projects are being progressed by our group based on the stars in this survey: studying the connection between various chromospheric activity indicators and the star formation process in the chromosphere (see some preliminary results in López-Santiago et al. 2005; Crespo-Chacón et al. 2005); and determining abundances of different elements in each star of the sample.

In this paper, we compile data derived by us for the stars observed in our survey. Our aim is to make the spectroscopic data accessible to the scientific community for future studies. In this new era of Virtual Observatory and extensive photometric databases and catalogs, the compilation of spectroscopic data is important for the purpose of constraining the properties of different astronomical objects, in particular, of stars (e.g. Sciortino et al. 1995; Takeda et al. 2007; Micela et al. 2007; López-Santiago et al. 2007; Klutsch et al. 2008; López-Santiago et al. 2009). The utility of substantial spectroscopic compilations has been demonstrated in the past by various groups. For instance, the Geneva-Copenhagen group derived metallicities, ages and kinematics from spectroscopic observations of a very large sample of $\mathrm{F}$ to K-type (FGK) stars of the solar neighborhood (Nordström et al. 2004). These data were then used to investigate relations between metallicity, kinematics and age in the context of the evolution of the Galactic disk (Nordström et al. 2004; Holmberg et al. 2007, 2009). Also, Fuhrmann (2004, 2008) determined spectroscopic parameters (temperature, gravity, metallicity, mass) of FGK stars in the solar vicinity with the aim of constructing an unbiassed sample of stars in the Galactic thin and thick disk. Similarly, Allende Prieto et al. (2004) assembled a catalog of metallicities of late-type stars less than $25 \mathrm{pc}$ from the sun.

Several spectroscopic surveys were performed mainly to study the evolution of magnetic activity with age in late-type stars, or simply to investigate chromospheric activity in general. Thus, the Palomar/MSU group compiled an extensive sample of nearby $M$ stars and determined both kinematics (Reid et al. 1995) and chromospheric activity (Hawley et al. 1996). The data were also used to study the star formation history and luminosity function of the solar neighborhood (Gizis et al. 2002; Reid et al. 2002). Another study on chromospheric emission is that of Rauscher \& Marcy (2006), who measured Ca II H \& K in a large sample of K7-M stars. Some groups are carrying out studies on how variations in line profiles produced by chromospheric activity affect the detection of planets. These studies are producing new catalogs of natural targets of planet searches with chromospheric emission measurements (e.g. Martínez-Arnáiz et al. 2010, and references therein).

Other surveys have specifically focused on determining spectroscopic parameters of active stars. For instance, Strassmeier et al. (2000) presented measurements of equivalent widths of $\mathrm{Ca}$ II $\mathrm{H} \& \mathrm{~K}, \mathrm{H} \alpha$, and $\mathrm{Li}$ I in addition to the kinematics of a substantial sample of active and inactive FGK stars. Also, Torres et al. (2006) and Guillout et al. (2009) determined kinematics and age indicators in comprehensive samples of late-type stars to select members of young moving groups and associations. White et al. (2007) determined the same parameters for stars in the Spitzer Legacy Science Program "The Formation and Evolution of Planetary Systems", aimed at studying the formation and evolution of protoplanetary disks. And Shkolnik et al. (2009) performed a spectroscopic survey of young M dwarfs within 25 pc. As Valenti \& Fischer (2005) demonstrated in their spectroscopic analysis of effective temperature, surface gravity, metallicity, projected rotational velocity and abundance of 1040 nearby FGK stars, the use of automated tools provides uniform results and makes the analysis of large samples practical.

We determine here equivalent widths and fluxes of most of the chromospheric activity indicators from $\mathrm{Ca}$ II $\mathrm{H} \& \mathrm{~K}$ to 
the Ca II infrared triplet (including the Balmer series and the $\mathrm{Na}$ I doublet and $\mathrm{Mg}$ I triplet) in a sample of stars of the spectral types F to $\mathrm{M}$. In this sense, our study implies an extension in terms of both spectral type range and wavelength coverage. We use the subtraction technique to subtract the photospheric contribution from the observed spectra, avoiding the use of calibrations to determine chromospheric emission. This approach represents an advance on previous studies. Together with the kinematics, rotation, and equivalent widths of Li I determined in this work, the sample constitutes an excellent laboratory for understanding the formation and evolution of the solar neighborhood during the past billion years.

The structure of the paper is as follows: in Sect. 2 we give details of the sample selection, the observations, and the data reduction methodology. In Sect. 3 we describe how we determined each parameter from the spectra. A brief summary of the results is given in Sect. 4. The Appendix contains tables with all the data and some figures with the spectra of stars in terms of selected chromospheric and photospheric features.

\section{Sample selection, observations and data reduction}

Our sample contains a total of 144 late-type stars. We selected 105 single stars from Montes et al. (2001a). Due to their membership in any of the moving groups studied in Montes et al. (2001a) (see Sect. 3.2 for a more detailed discussion on the stellar kinematic groups), we were quite certain that they are young and present chromospheric emission lines. Although latetype $\mathrm{K}$ and $\mathrm{M}$ stars remain active for a long period of their life (e.g. West et al. 2008), adding the condition of being member of a young moving group places a greater restriction on a star's age. Nevertheless, since the Montes et al. (2001a) sample also contains some old stars (López-Santiago et al. 2009), several stars of our present work could be older than $\sim 1$ Gyr. The remaining 39 stars of our sample were selected because they showed a high level of magnetic activity, rotational rate, and/or lithium abundance. A complete list of the stars observed by us is given in Table 1 . The sample is restricted in declination since the observations were taken in telescopes sited in the Northern Hemisphere. The minimum declination reached is approximately $-20^{\circ}$. Another restriction is the brightness of the star. Since echelle spectrographs have low efficiency, only stars with approximately $V \leq 12$ mag were selected. Our aim was to obtain spectra with high $S / N$ even for the faintest stars (70-200 in the region of $\mathrm{H} \alpha$ ). Spectral-type reference stars and radial velocity standards, used to determine the chromospheric excesses and heliocentric velocities of our stars, were also observed in each campaign. To obtain robust results, we ensured good spectral-type coverage in each observing run. A complete list of these stars is given in Table A.1.

The observations were carried out during twelve observing runs between 1999 and 2002. We used high resolution echelle spectrographs (resolving power, $\lambda / \Delta \lambda$, ranging from 30000 to 60000 at $6500 \AA, \Delta \lambda \sim 0.15 \AA$ ), with the exception of one observing run, where we used a long-slit spectrograph with spectral resolution $\Delta \lambda=1.13 \AA$. Eighteen stars were observed with the latter configuration, nine of them were observed only during this observing run. For these nine stars, we determined only radial and rotational velocities and measured equivalent widths of Li I when possible. In general, errors in the measurements are only slightly larger for these stars than for those observed with higher resolution (see Table A.2). However, the low resolution of the spectra in this observing run prevented us from measuring chromospheric emission in these nine stars to compare with our high resolution spectra (see Walkowicz \& Hawley 2009, for a discussion on this issue). Details of each observing run are given in Table 2: date, telescope, spectrograph, CCD chip, spectral range covered, number of orders included in each echelle spectrum, range of reciprocal dispersion and spectral resolution (determined as the full width at half maximum, $F W H M$, of the arc comparison lines). Some of the stars were observed more than once (in the same campaign or even in different ones). The total number of spectra collected for this survey is 518 for targets and more than 50 for standards.

For data reduction, we used the standard procedures in the IRAF $^{1}$ package (bias subtraction, extraction of scattered light produced by optical system, division by a normalized flat-field and wavelength calibration). After reduction, each spectrum was normalized to its continuum, order by order, by fitting a polynomial function.

\section{Results}

\subsection{Radial velocities}

Heliocentric radial velocities were determined with the crosscorrelation technique. In each observing run, the spectrum of each star was cross-correlated order by order against spectra of radial velocity standards of similar spectral type (stars marked with an asterisk in Table A.1) with the routine fxcor in IRAF. For each observed spectrum, radial velocities were derived for different spectral orders from the position of the peak of the cross-correlation function (CCF) by fitting a Gaussian to the function. Then, weighted means were calculated with the individual values obtained for each spectral order. To avoid systematic errors produced by the effect of cool spots in the CCF, we fitted the Gaussian to the entire CCF profile, instead of fitting only the peak.

Our results are listed in Table A.2. We give radial velocities for each observation of the star $\left(V_{\mathrm{r}}\right)$ and a mean velocity determined from the individual results for each observation $\left(\bar{V}_{\mathrm{r}}\right)$. Although our sample was selected from a list of single stars, some of them are actually single-line spectroscopic binaries. Known binaries in our sample are: HD 16525 , HD 17190, HD 17382, HD 140913, HD 167605, HIP $89874^{2}$ and HD 208472. During our observations, other stars presented variations in their radial velocities that were hardly attributable to spots. If caused by spots, they should produce noticeable asymmetries in the absorption line profiles, which was not observed in their spectra. For other stars, we determined radial velocities that were quite different from those given in the literature. Therefore, we classified all these stars as possible binaries: BD+28 1779, HD 85270, GJ 466, HD 112542, HD 112733 , HD 238224, HD 160934, BD-05 5480, and GJ 842.2. The star HD 160934 was confirmed as a binary system by Hormuth et al. (2009) with preliminary orbital parameters determined by Gálvez et al. (2006).

Variations in the radial velocity measurements of up to several kilometers per second are induced in the CCF by assymetries caused by spots on the stellar disk. This was even

\footnotetext{
${ }^{1}$ IRAF is distributed by the National Optical Observatory, which is operated by the Association of Universities for Research in Astronomy, Inc., under contract with the National Science Foundation.

${ }^{2}$ HIP 89874 (FK Ser) is a binary with a separation of 1.33 arcsec (Herbig \& Bell 1988; Jensen et al. 1996). In our spectra, we were not able to separate the two components.
} 
Table 1. Late-type stars studied in this work (HD number or other name).

\begin{tabular}{|c|c|c|c|c|c|c|c|c|}
\hline 166 & 1405 & 1326 & 1835 & 2410 & QT And & 4568 & 4614 & $4614 \mathrm{~B}$ \\
\hline $\mathrm{BD}+17232$ & 12230 & 13382 & 16525 & 17190 & 17382 & 17925 & 17922 & 18632 \\
\hline 18803 & 20678 & 21845 & 23232 & 24916 & 25457 & 25680 & 25998 & 25665 \\
\hline 29697 & 30652 & 33564 & 36869 & 37394 & 233153 & 41593 & TYC $1355-75-1$ & $\mathrm{BD}+201790$ \\
\hline HIP 39721 & GJ 9251B & HIP 39896 & 72905 & 73171 & 77191 & 77407 & 82558 & 82443 \\
\hline HIP 47176 & HIP 49544 & HIP 50156 & GJ 388 & HIP 51317 & 85270 & 98736 & GJ 426B & 102392 \\
\hline 105631 & 238087 & 238090 & 106496 & HIP 60661 & 110010 & HIP 62686 & HIP 63023 & 112542 \\
\hline 112733 & 115043 & HIP 65016 & 238224 & 117860 & HIP 67092 & 125161B & 129333 & 133826 \\
\hline 134319 & 135363 & 140913 & 142764 & 143809 & 145675 & 146696 & 147379A & 147379B \\
\hline HIP 79796 & 149661 & 149931 & 152863 & 152751 & $155674 \mathrm{~A}$ & 155674B & 156984 & HIP 84794 \\
\hline HIP 85665 & 160934 & 162283 & HIP 87579 & HIP 87768 & GJ 698B & 165341 & GJ 702B & 167605 \\
\hline 234601 & SAO 9067 & 168442 & HIP 89874 & 171488 & 171746 & 173739 & 173740 & 2RE J1846+191 \\
\hline GJ 734B & 184525 & 187458 & 187565 & 191011 & HIP 101262 & 197039 & HIP 102401 & 198550 \\
\hline 200560 & 200740 & 201651 & HIP 104383 & EUVE J $2113+04.2$ & HIP 105885 & HIP 106231 & 205435 & 206860 \\
\hline 208472 & HIP 108467 & HIP 108752 & TYC1680-01993-1 & 209458 & HIP 109388 & V383 Lac & GJ 856B & 213845 \\
\hline $\mathrm{BD}+174799$ & HIP 112460 & 216899 & 217813 & HIP 114066 & 220140 & 221503 & HIP 117779 & HIP 118212 \\
\hline
\end{tabular}

Table 2. Observing run details.

\begin{tabular}{|c|c|c|c|c|c|c|c|c|}
\hline Id. & Date & Telescope & Instrument & CCD chip & $\begin{array}{c}\text { Spectral range } \\
(\AA)\end{array}$ & Orders & $\begin{array}{c}\text { Dispersion } \\
(\AA)\end{array}$ & $\begin{array}{c}F W H M \\
(\AA)\end{array}$ \\
\hline 1 & 1999 Jul. 24-29 & $2.2 \mathrm{~m}^{a}$ & FOCES $^{1}$ & $2048 \times 204815 \mu \mathrm{m}$ LORAL\#11 & $3910-9075$ & 84 & $0.03-0.07$ & $0.09-0.15$ \\
\hline 2 & 1999 Nov. 26-27 & $\mathrm{NOT}^{b}$ & SOFIN $^{2}$ & $1152 \times 770$ EEV P88200 & $3525-10425$ & 44 & $0.06-0.17$ & $0.14-0.32$ \\
\hline 3 & 2000 Jan. 18-22 & $\mathrm{INT}^{c}$ & MUSICOS $^{3}$ & $1024 \times 102424 \mu \mathrm{m}$ TEK 5 & $4430-10225$ & 73 & $0.07-0.15$ & $0.16-0.30$ \\
\hline 4 & 2000 Aug. 05-11 & $\mathrm{INT}^{c}$ & MUSICOS $^{3}$ & $1024 \times 102424 \mu \mathrm{m}$ TEK5 & $4430-10225$ & 73 & $0.07-0.15$ & $0.16-0.30$ \\
\hline 5 & 2000 Nov. $10-13$ & $\mathrm{NOT}^{b}$ & SOFIN $^{2}$ & $1152 \times 770$ EEV P88200 & $3525-10425$ & 44 & $0.06-0.17$ & $0.14-0.32$ \\
\hline 6 & 2001 Apr. 02-05 & $\mathrm{INT}^{c}$ & $\mathrm{IDS}^{4}$ & $2148 \times 420013.5 \mu \mathrm{m}$ EEV10a & $3554-7137$ & 1 & 0.48 & 1.22 \\
\hline 7 & 2001 Sep. 21-24 & $2.2 \mathrm{~m}^{a}$ & FOCES $^{1}$ & $2048 \times 204824 \mu$ m Site\#1d & $3510-10700$ & 112 & $0.04-0.13$ & $0.08-0.35$ \\
\hline 8 & 2001 Oct. $10-11$ & $\mathrm{TNG}^{d}$ & SARG $^{5}$ & $2(2048 \times 4096) 13.5 \mu \mathrm{m}$ EEV 4280 & $4960-10110$ & 62 & $0.02-0.04$ & $0.08-0.17$ \\
\hline 9 & $\begin{array}{l}2001 \text { Dec. } 19- \\
2002 \text { Feb. } 28\end{array}$ & $\mathrm{HET}^{e}$ & $\mathrm{HRS}^{6}$ & $2(2048 \times 4096) 15 \mu \mathrm{m}$ Marconi & $5040-8775$ & 52 & $0.06-0.11$ & $0.15-0.28$ \\
\hline 10 & 2002 Apr. 22-25 & $2.2 \mathrm{~m}^{a}$ & FOCES $^{1}$ & $2048 \times 204824 \mu \mathrm{m}$ Site\#1d & $3510-10700$ & 112 & $0.04-0.13$ & $0.08-0.35$ \\
\hline 11 & 2002 Jul. 01-06 & $2.2 \mathrm{~m}^{a}$ & FOCES $^{1}$ & $2048 \times 204824 \mu \mathrm{m}$ Site\#1d & $3510-10700$ & 112 & $0.04-0.13$ & $0.08-0.35$ \\
\hline 12 & 2002 Aug. 21-29 & $\mathrm{NOT}^{b}$ & SOFIN $^{2}$ & $2048 \times 2048$ 2K3EB PISKUNOV1 & $3525-10200$ & 42 & $0.02-0.05$ & $0.05-0.15$ \\
\hline
\end{tabular}

Notes. ${ }^{(a)} 2.2 \mathrm{~m}$ telescope at German Spanish Astronomical Observatory (CAHA) (Almería, Spain); ${ }^{(b)} 2.56 \mathrm{~m}$ Nordic Optical Telescope (NOT) at Observatorio del Roque de los Muchachos (La Palma, Spain); ${ }^{(c)} 2.5 \mathrm{~m}$ Isaac Newton Telescope (INT) at Observatorio del Roque de los Muchachos (La Palma, Spain); ${ }^{\left({ }^{d}\right)} 3.5 \mathrm{~m}$ Telescopio Nazionale Galileo (TNG) at Observatorio del Roque de los Muchachos (La Palma, Spain); ${ }^{(e)} 9.2 \mathrm{~m}$ HobbyEberly Telescope (HET) at McDonald Observatory (Texas, USA).

(1) FOCES: Fiber Optics Cassegrain Echelle Spectrograph; ${ }^{(2)}$ SOFIN: Soviet Finnish High Resolution Echelle Spectrograph; ${ }^{(3)}$ MUSICOS: spectrograph developed as part of MUlti-SIte COntinuous Spectroscopy project; ${ }^{(4)}$ IDS: Intermediate Dispersion Spectrograph; ${ }^{(5)}$ SARG: Spettrografo di Alta Resoluzione Galileo; ${ }^{(6)}$ HRS: High Resolution Spectrograph.

then the case when a fit to the entire cross-correlation profile was performed (Dempsey et al. 1992; Strassmeier et al. 2000; López-Santiago et al. 2003). We observed variations of this order for several stars in our sample for which we took spectra during different nights of the same observing run: HD 1405, BD+17 232, BD+20 1790, HD 72905, HD 82558, AD Leo, HD 135363, HD 171488, V383 Lac and HD 220140.

In Table A. 2 we also give the photometric periods available in the literature for some of the stars in the sample.

\subsection{Space motion}

Galactic space-velocity components $(U, V, W)$ were determined as in Montes et al. (2001a), who used a modified version of the original procedure of Johnson \& Soderblom (1987) to calculate Galactic velocities and associated uncertainties. As in Montes et al. (2001a), we did not correct $(U, V, W)$ for solar motion to make comparisons with other works regarding moving groups easier. We used Hipparcos and Tycho-2 data (ESA 1997; Høg et al. 2000) and radial velocities determined by us.
For the ten stars with no available distance measurements in the literature, we determined a spectroscopic parallax using information on spectral type and luminosity class from our spectra. The Schmidt-Kaler (1982) color-magnitude relations were used to determine $M_{V}$ for these stars. Note that for late-K and M stars, classic relations are not appropriate for determining some observed quantities. In particular, better spectroscopic parallax relations have been developed in the literature using molecular bands (e.g. Bochanski et al. 2005). Nevertheless, the spectral types of the stars in our sample for which we have determined a spectroscopic parallax are in the range F5-K3. Note also that small variations in the parallax $(<10$ mas) of stars produce only small variations in the Galactic velocities $\left(<0.1 \mathrm{~km} \mathrm{~s}^{-1}\right)$.

The resultant $U, V$ and $W$ velocity components are listed in Table A.2. We used the mean radial velocity determined by us ( $\bar{V}_{\mathrm{r}}$ in the table) for this computation. For the possible binary systems, we did not correct for binarity, because their orbits are as yet unknown. Thus, we used the observed radial velocities - or the mean value when more than one observation was performed - as a first approximation. 
As we mentioned in Sect. 2, most of the stars in our sample (105) were selected from Montes et al. (2001a), who give a list of members and possible members of the young moving groups. For these, we obtained Galactic velocity components very similar to those given in Montes et al. (2001a). The remaining 39 stars of our sample had no previous measurement of $U V W$ velocities. As a first estimate from their position in the $U V$ - and $W V$-plane, 50 stars could be classified as members or possible members of the Local Association (in its various subgroups), 25 of the Hyades Supercluster, 20 of the Ursa Major Moving Group, 9 of the IC 2391 Supercluster, 5 of the Castor Moving Group, and the other 17 as young disk stars with no clear membership (see Table A.2). The same velocity dispersion as in Montes et al. (2001a) was used for determining the membership of the stars in any of the moving groups. We refer the reader to that paper for a detailed explanation. Surprisingly, 18 stars are well outside the classical boundaries of the young disk population in the $U V$-plane. Most of them are the lowest active stars in our sample, but two of them are very active stars: HIP 79796 and HD 216899 (see Figs. A.1-A.4).

\subsection{Rotational velocities}

To determine the rotational velocities in our sample, we used a methodology based on the cross-correlation technique, as we did for radial velocities (see Soderblom et al. 1989, for a detailed discussion). The width of the peak of the CCF depends on the physical processes contributing to the line profile. The mathematical concept is very similar to that of the convolution of a theoretical spectrum with a rotation profile (see Gray 2005, for details). But, instead of comparing the stellar spectrum with a rotationally broadened one, the cross-correlation is performed between the spectrum of the program star and a non-rotating one observed with the same instrument. Best results are obtained when both the comparison and the program stars have similar spectral types.

For each reference (non-rotating) star observed with our sample we first calibrated the relation between the CCF width and $v \sin i$ value by cross-correlating the star with itself after rotationally broadening its spectrum at different velocities (with values ranging from 1 to $60 \mathrm{~km} \mathrm{~s}^{-1}$ ). The result is a relation between CCF width and rotational velocity (see Fig. 1). The relation depends on the spectral type of the star (as shown in the figure). Then we cross-correlated the program star with a standard with similar spectral type and used the relation for this standard to determine the rotational velocity of the program star. Since the relation also depends on the instrumental configuration, the cross-correlation was performed separately in each observing run. A detailed explanation of the method can be found in López-Santiago et al. (2003). For our study, the CCF peak width was determined by fitting a Gaussian function to it. This method ensures good results for $v \sin i \leq 50 \mathrm{~km} \mathrm{~s}^{-1}$ (Soderblom et al. 1989). In our sample, there is only one star with $v \sin i>$ $50 \mathrm{~km} \mathrm{~s}^{-1}$ (LO Peg). For this star, we determined its rotational velocity by comparison with artificially broadened spectra of different stars with similar spectral types.

In Table A.2, we give each value of $v \sin i$ obtained for the stars in the sample and a mean value determined from the individual results. Uncertainties were determined with the parameter $R$ defined by Tonry \& Davis (1979) as the ratio of CCF height to the rms antisymmetric component. This parameter is computed by fxcor and provides a measurement of the signal-to-noise ratio of the CCF. Tonry \& Davis (1979) showed that errors in the CCF width are proportional to $(1+R)^{-1}$,

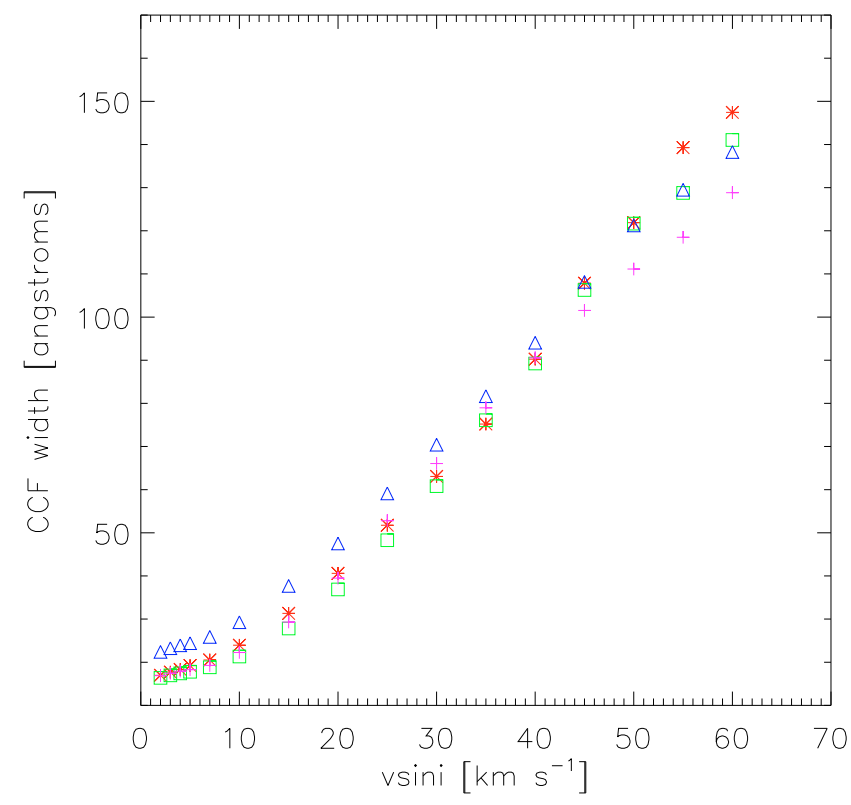

Fig. 1. CCF width $-v \sin i$ relation for standard stars of different spectral type in the same observing run: HD 182488 (G8V, asterisks), HD 185144 (K0 V, squares), HD 166620 (K2V, plusses), and HD 201091 (K5V, triangles).

while Hartmann et al. (1986) and Rhode et al. (2001) found that the quantity $\pm v \sin i(1+R)^{-1}$ provides a good estimate for the $90 \%$ confidence level of the $v \sin i$ measurement. Thus we adopted $\pm v \sin i(1+R)^{-1}$ as a reasonable estimate of the uncertainties in our determinations.

\subsection{Spectral types and the lithium line}

During each observing run, a number of spectral-type standards were observed, covering the range of spectral types in our sample (from $\mathrm{F}$ to $\mathrm{M}$ ). To determine spectral types, we performed fits of our sample stars to spectral-type standards with a modified version of STARMOD (Barden 1985, see Sect. 3.5 for a detailed description of the procedure). The software first rotationally broadens the spectrum of the standard star until the best fit is obtained. Then it subtracts the obtained synthetic spectrum from the sample star. In theory, if both stars have the same spectral type, the resultant (subtracted) spectrum should be null. In practice, the subtracted spectrum shows some noise, due to small differences in metallicity and/or gravity and also when the $\mathrm{S} / \mathrm{N}$ of one of the spectra is low. Nevertheless, small differences in metallicity and gravity are lower than those produced by the difference of one spectral subtype. The procedure of fitting is repeated with each of the standard stars until the best result is obtained. Errors are estimated in one spectral subtype.

Lines sensitive to spectral type were also used to determine spectral type. In particular, we used the lines Fe I $\lambda 6430 \AA$, Fe II $\lambda 6432$ and $6457 \AA$, Ca I $\lambda 6449$ and $6456 \AA$, Co I $\lambda 6455 \AA$, and V I $\lambda 6452 \AA$, as described in Strassmeier \& Fekel (1990). Other spectral lines used in this work for spectral classification are the $\mathrm{Mg}$ I triplet $\lambda 5167,5172$, and $5183 \AA$, the Na I doublet $\lambda 5590$ and $5596 \AA$, Ca I $\lambda 6573 \AA$ and Fe I $\lambda 6575 \AA$. In contrast to the subtraction technique, this method is suitable only for slow rotators, since the lines involved in each relation of Strassmeier \& Fekel (1990) are blended in stars with high rotational velocities. Note that the relations are calibrated only for 


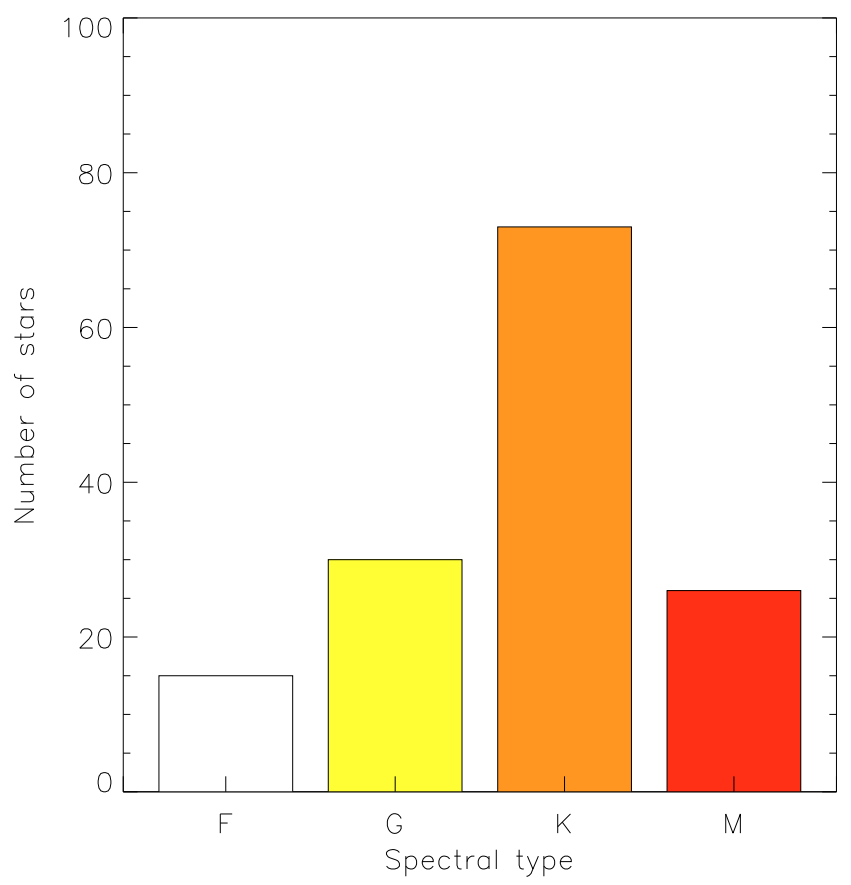

Fig. 2. Spectral type distribution of the stars in the sample.

FGK stars, but not for $\mathrm{M}$ ones. We used this method to test the results obtained with the subtraction technique for the stars in our sample showing small values of $v \sin i$. The most significant differences are two spectral subtypes for F stars and one subtype for $\mathrm{G}$ and $\mathrm{K}$ stars. These values are inside the uncertainties of the relations of Strassmeier \& Fekel (1990). Our final results are given in Table A.2 (see also Fig. 2).

We measured the equivalent width of the lithium line at $6707.8 \AA$ in the observed spectra. The lithium abundance is an appropriate age indicator for approximately log age $\leq 8.8 \mathrm{Myr}$ (the age of the Hyades cluster) because this element is easily destroyed by thermonuclear reactions in the stellar interior. Thus detection of the lithium line is generally a sign of stellar youth in single stars (high rotation velocities could preserve the lithium from depletion over longer time scales in binaries). The lithiumage relation is mass-dependent. The usual way to determine the age range is by comparison with stars of similar spectral type in clusters of a well-known age (e.g. Soderblom \& Mayor 1993; Neuhaeuser et al. 1997; Montes et al. 2001b). In our spectra, the $\mathrm{Li}$ I $\lambda 6707.8 \AA$ line is blended with the Fe I $\lambda 6707.4 \AA$ line. To correct the measured equivalent width $(E W[\mathrm{Li}$ I $+\mathrm{Fe} \mathrm{I}])$ for the Fe I line, we used the empirical relationship of Soderblom et al. (1993). The results are given in Table A.2. An error-weighted mean value of the individual $E W[\mathrm{Li}$ I] measured on different nights and over different observing runs was also determined (see Table A.2). Many stars in the sample still show the Li I absorption line in their spectra (see Table A.2 and Fig. A.3). Some of them are very young (log age $\leq 7.5 \mathrm{Myr}$ ). They belong to the young stellar associations (Zuckerman et al. 2004; Torres et al. 2008). In particular, López-Santiago et al. (2006) established different age subgroups in the AB Dor moving group using results from this study.

\subsection{Chromospheric activity}

A special feature of echelle spectrographs is that they cover a large fraction of the optical spectrum. It allows simultaneous observations of all the activity indicators in this spectral range to be obtained. We measured equivalent widths of the chromospheric optical lines Ca II $\mathrm{H} \& \mathrm{~K}, \mathrm{H} \epsilon, \mathrm{H} \delta, \mathrm{H} \gamma, \mathrm{H} \beta, \mathrm{H} \alpha$, and the $\mathrm{Ca}$ II infrared triplet, for each observation of each star in our sample, when the spectrograph configuration permitted it. In very active stars (active $\mathrm{M}$ dwarfs and flare stars), we also measured equivalent widths of the lines He I $\lambda 5876 \AA$ and the Na I doublet

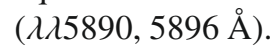

To remove the photospheric contribution, we used the spectral subtraction technique (see details in Montes et al. 2000). The advantage of this method is that no assumption about the continuum value for the equivalent width measurement is needed. Reference (non-active) stars with similar spectral types to our targets were taken as templates (see Table A.1). The subtraction was performed with JSTARMOD, a modified version of the Fortran code STARMOD developed at the Pennsylvania State University (Huenemoerder \& Barden 1984; Barden 1985). Our modifications permit the program to use echelle spectra in the file format given by the majority of observatories. JSTARMOD first selects the region of the spectrum indicated by the user. Then it rotationally broadens and shifts the template spectrum to fit the target one. Finally it subtracts the synthetic spectrum from the observed one. If both stars - active and non-active - are identical in terms of photosphere, the resultant subtracted spectrum is the chromospheric emission of the active star, i.e. a flat spectrum with emission features at the positions of the chromospheric lines. In practice, the subtracted spectrum shows some noise away from the chromospheric lines, due to small differences in metallicity and/or gravity.

A source of uncertainties is the possible basal emission of the non-active stars used as templates. Its consequence is a reduction in the measured equivalent widths of the Ca II H \& $\mathrm{K}$ chromospheric emissions of the target. However, for the subtraction we used reference stars situated close to the lower boundary of the surface flux in Ca II H \& K of Rutten (1984). We estimate the largest uncertainty in logarithm fluxes as $0.1 \mathrm{dex}$, due to basal chromospheric emission from standard non-active stars.

The equivalent widths were determined by fitting a Gaussian function to the emission line profiles. For the Ca II $\mathrm{H}$ and $\mathrm{H} \epsilon$ lines, which are blended in our spectra, we used a double Gaussian fit. To obtain an estimation of the errors, we followed the methodology explained in López-Santiago et al. (2003). Our results are given in Table A.3. Each measurement of the equivalent width and its uncertainty is listed in the table, together with the observing run and the Modified Julian Date (MJD) of the observation. Equivalent widths were later converted to absolute chromospheric fluxes at the stellar surface with the calibrations of Hall (1996) (see results in Table A.4).

In Fig. 3 we show the distribution of fluxes in different chromospheric lines (to allow statistical comparisons with other works). The sample has a peak towards high chromospheric fluxes in chromospheric calcium. Two peaks are observed in $\mathrm{H} \alpha$. The peak at high fluxes in each line is a consequence of the selection method. The double peak in $\mathrm{H} \alpha$ is presumably caused by the presence of two populations of $\mathrm{H} \alpha$ emitters: saturated and non-saturated. To verify this hypothesis, we constructed plots of the ratio $F_{\text {line }} / F_{\text {bol }}$ versus temperature for each chromospheric line. In Fig. 4, we show the result for $\mathrm{H} \alpha$ and the Ca II $\lambda 8542 \AA$ (IRT2) line. Two well-defined branches are observed for $\mathrm{H} \alpha$. A similar behavior was observed for coronal sources (e.g. López-Santiago et al. 2009) and was attributed to $\mathrm{X}$-ray emission saturation. Similarly, the stars in our sample show saturation in $\mathrm{H} \alpha$ at a mean value $\log F_{\mathrm{H} \alpha} / F_{\text {bol }} \sim-3.8$, 

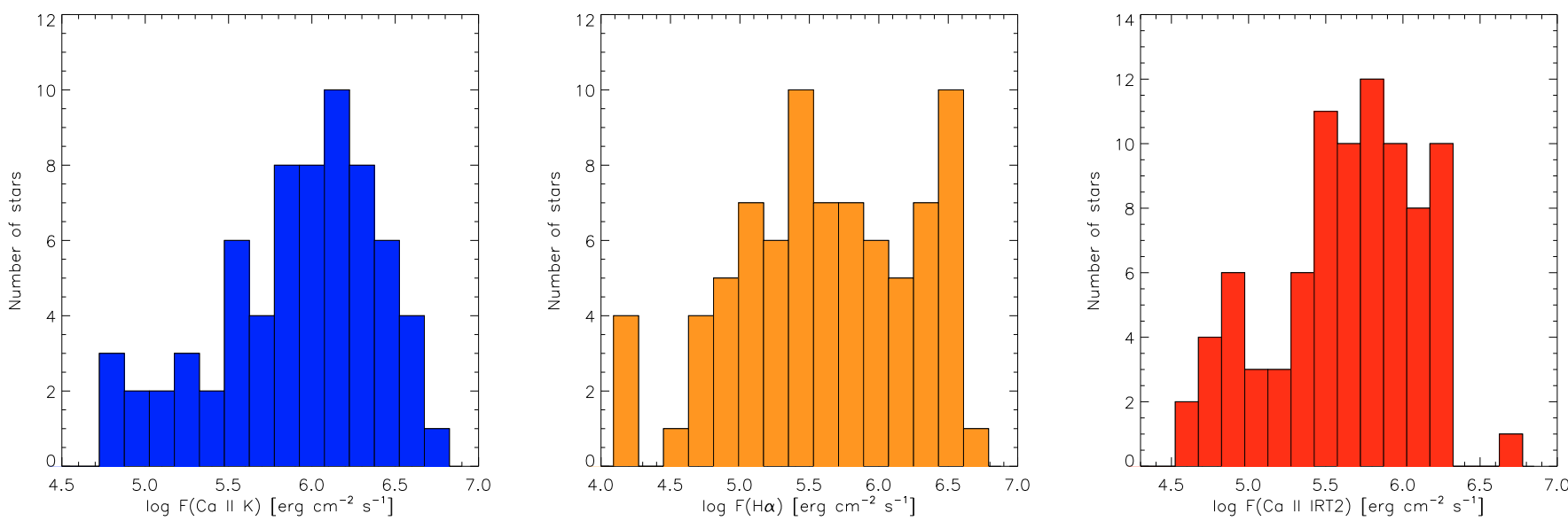

Fig. 3. Histograms for results on some chromospheric activity indicators (absolute flux after subtraction of the photosphere).
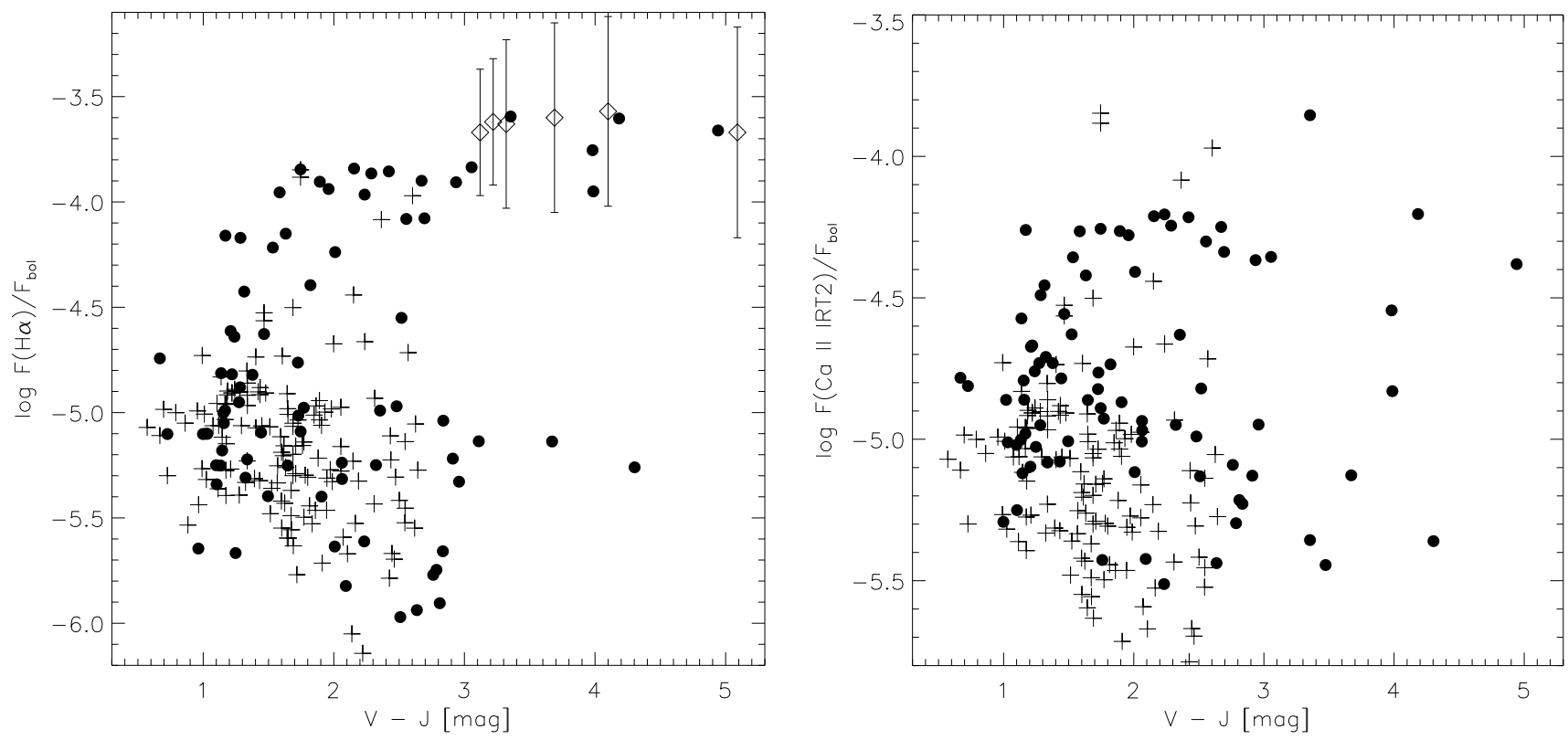

Fig. 4. Left: $\log F_{\mathrm{S}}\left(\mathrm{H}_{\alpha}\right) / F_{\text {bol }}$ versus $V-J$ for the stars in our sample (dots). Plusses are the stars in Martínez-Arnáiz et al. (2010). Diamonds are the data of West et al. (2004). Right: $\log F_{\mathrm{S}}(\mathrm{Ca}$ II $\lambda 8542 \AA) / F_{\text {bol }}$ versus $V-J$ of the stars in our sample. Symbols are the same as in the left figure.

as was found for early to mid-M stars (e.g. Walkowicz et al. 2004; West et al. 2008). The effect of saturation is less marked in other indicators, like the $\mathrm{Ca}$ II lines (see Fig. 4, right).

In addition to the absolute fluxes in the different chromospheric lines we also determined the $R_{\mathrm{HK}}^{\prime}$ index (see Table A.4), which is defined as the ratio of the emission from the chromosphere in the $\mathrm{Ca}$ II $\mathrm{H} \& \mathrm{~K}$ lines to the total bolometric emission of the star, i.e.

$R_{\mathrm{HK}}^{\prime}=\frac{F_{\mathrm{H}}^{\prime}+F_{\mathrm{K}}^{\prime}}{\sigma T^{4}}$

where $F_{\mathrm{H}}^{\prime}$ and $F_{\mathrm{K}}^{\prime}$ are the chromospheric fluxes in the $\mathrm{Ca}$ II $\mathrm{H}$ and $\mathrm{K}$ lines, respectively. Effective temperatures were determined through empirical calibrations with the color index $B-V$ (e.g. Gray 2005). These calibrations are valid for $B-V \leq 1.5$. Only seven stars of our sample (three of them being giants) have values of $B-V$ above 1.5. For these, we extrapolated the colortemperature relation. For $B-V>1.5$, the spread in temperatures is wide (see Fig. 14.6 in Gray 2005). Differences between the value given by the relation and that obtained with other methods (e.g. Flower 1996) of up to 100-200 K are observed for M dwarfs.

Figures A.1-A.3 show the spectra of the stars in the sample in spectral regions containing the $\mathrm{Ca}$ II $\mathrm{K}$ line, $\mathrm{H} \alpha$, and part of the Ca II infrared triplet.

\subsection{Summary}

Radial and rotational velocities were derived from each observation of a star in our sample with the cross-correlation technique. We derived mean radial and rotational velocities for each star. In some cases (see Sect. 3.1) we observed significant variations in the radial velocity of the star, which we attributed to binarity. Mean values of radial velocities were used to derive space motions. We also determined spectral types from the spectra of stars. Table A. 2 summarizes these results.

With regard to spectral lines, we determined equivalent widths of all optical chromospheric activity indicators of each star as well as the Li I $\lambda 6708 \AA$ line (see Table A. 2 for results of the Li I line). To reveal chromospheric emission lines in the 
spectra, we used the spectral subtraction technique. Non-active stars with spectral types similar to those of our targets were taken as templates for the subtraction. Their spectra were conveniently broadened and shifted to fit our targets (see Sect. 3.4). The equivalent widths of the emission lines were converted into flux using equivalent width-flux relations. Tables A.3 and A.4 summarize the results of the spectroscopic survey.

For completeness, we performed a simple statistical study of our results on chromospheric activity indicators. In our sample, a large spread in fluxes is observed for the different activity indicators. The spread is especially noticeable for the $\mathrm{H} \alpha$ line (Fig. 3, middle). To investigate this finding, we analyzed the $F_{\mathrm{H} \alpha} / F_{\text {bol }}$ and $F_{\text {CaII }} / F_{\text {bol }}$ ratios as a function of the color of the stars. The results indicate the presence of two populations of chromospherically active stars in our sample. In the $\log F_{\mathrm{H} \alpha} / F_{\text {bol }}-$ $(V-J)$ diagram (Fig. 4, left), two branches are clearly observed for $V-J \geq 1.4 \mathrm{mag}$ (corresponding to an early-K dwarf). This dichotomy is statistically significant. Out of the 79 stars in our sample with chromospheric $\mathrm{H} \alpha$ emission, 53 have $V-J>1.4$ with 23 stars in the upper branch and the remaining 30 in the lower one. The two groups have mean values $\log F_{\mathrm{H} \alpha}=-3.9$ with variance 0.04 and $\log F_{\mathrm{H} \alpha}=-5.3$ with variance 0.13 , respectively. A simple two-sample t-test (e.g. Snedecor \& Cochran 1989) assures that the two means are different (i.e. the two branches are statistically different), with a significance of 0.01 (corresponding to a probability of $99 \%$ ). The presence of the two branches is less clear when using other chromospheric activity indicators (see Fig. 4, right).

In a recent work, López-Santiago et al. (2009) demonstrated that the sample of possible members of the young stellar kinematic groups of Montes et al. (2001a) is partially contaminated by active field stars that do not belong to the moving groups. In that work, the stars in the X-ray saturation regime also showed high $\mathrm{H} \alpha$ fluxes. In their Fig. 4, López-Santiago et al. (2009) observed two branches: one for the high $\mathrm{H} \alpha$ emitters and the other for the remaining active stars. Those stars populating the upper branch were indeed young ( 10-120 Myr). In contrast, field stars populated the lower branch. The study of López-Santiago et al. (2009) was performed using part of the data presented by us in this work. Therefore their results on the nature of the two populations of chromospheric active stars are applicable here. In fact, the stars in the top branch in Fig. 4 (left) are known to be young stars (see López-Santiago et al. 2006) and very active M dwarfs. A similar conclusion was already reached by Vaughan \& Preston (1980) for G and K stars. In their study, the authors observed two branches in the $\log S-(B-V)$ diagram with a gap between them. The authors called the active one the "young branch" and the less active one the "old branch". Recent results show that this dichotomy between very active stars and less active ones is also present in M dwarfs. In Fig. 4 (left), we overplotted the data obtained by West et al. (2004) for the active M stars in the SLOAN Digital Sky Survey (the dispersion observed by the authors for each sub-spectral type is represented by vertical bars). Although our method for measuring the line flux is slightly different from that of West et al. (2004), the figure clearly shows that $\mathrm{M}$ active dwarfs are located over the young active branch. A similar trend can be observed for the early M dwarfs of Reiners (2007). In general, these results suggest that $\mathrm{H} \alpha$ saturates equally in $\mathrm{K}$ to $\mathrm{M}$ stars.

Acknowledgements. Part of this work was co-supported by the Spanish MICINN, Ministerio de Ciencia e Innovación project numbers: AYA200800695 and AYA2008-06423-C03-03. J. López-Santiago is a postdoctoral fellow of the ASTROCAM (Red de Astrofísica en la CAM) with project number S-0505/ESP/000237 of the IV PRICIT Plan Regional de Investigación
Científica e Innovación Tecnológica de la Comunidad de Madrid. The authors would like to thank the referee for useful comments and suggestions that allowed us to improve our work.

\section{References}

Allende Prieto, C., Barklem, P. S., Lambert, D. L., \& Cunha, K. 2004, A\&A, 420, 183

Antoja, T., Figueras, F., Fernández, D., \& Torra, J. 2008, A\&A, 490, 135

Antoja, T., Valenzuela, O., Pichardo, B., et al. 2009, ApJ, 700, L78

Asiain, R., Figueras, F., Torra, J., \& Chen, B. 1999, A\&A, 341, 427

Barden, S. C. 1985 , ApJ, 295, 162

Barnes, T. G., III, Moffett, T. J., \& Slovak, M. H. 1986, PASP, 98, 223

Beavers, W. I., Eitter, J. J., Ketelsen, D. A., \& Oesper, D. A. 1979, PASP, 91, 698 Benz, W., \& Mayor, M. 1984, A\&A, 138, 183

Bochanski, J. J., Hawley, S. L., Reid, I. N., et al. 2005, AJ, 130, 1871

Bochanski, J. J., Munn, J. A., Hawley, S. L., et al. 2007, AJ, 134, 2418

Böhm-Vitense, E. 2007, ApJ, 657, 486

De Medeiros, J. R., \& Mayor, M. 1999, A\&AS, 139, 433

Crespo-Chacón, I., Montes, D., Fernández-Figueroa, M. J., \& López-Santiago, J.

2005, in Proceedings of the 13th Cambridge Workshop on Cool Stars, Stellar

Systems and the Sun, 5-9 July, 2004, Hamburg, Germany, ed. F. Favata,

G. A. J. Hussain, \& B. Battrick, European Space Agency, ESA SP-560, 491

De Medeiros, J. R., Do Nascimento, J. D., Jr., Sankarankutty, S., Costa, J. M., \&

Maia, M. R. G. 2000, A\&A, 363, 239

Dempsey, R. C., Bopp, B. W., Strassmeier, K. G., et al. 1992, ApJ, 392, 187

Dufflot, M., Figon, P., \& Meyssonnier, N. 1995, A\&AS, 114, 269

Dyer, E. R., Jr. 1954, AJ, 59, 218

ESA 1997, The Hipparcos and Tycho Catalogues, ESA SP-1200

Famaey, B., Jorissen, A., Luri, X., et al. 2005, A\&A, 430, 165

Famaey, B., Pont, F., Luri, X., et al. 2007, A\&A, 461, 957

Fekel, F. C. 1997, PASP, 109, 514

Flower, P. J. 1996, ApJ, 469, 355

Fuhrmann, K. 2004, Astron. Nachr., 325, 3

Fuhrmann, K. 2008, MNRAS, 384, 173

Gálvez, M. C., Montes, D., Fernández-Figueroa, M. J., \& López-Santiago, J. 2006, Ap\&SS, 304, 59

Gray, C. D. F. 2005, Stellar Photospheres (Cambridge: Cambridge University Press)

Guillout, P., Klutsch, A., Frasca, A., et al. 2009, A\&A, 504, 829

Gizis, J. E., Reid, I. N., \& Hawley, S. L. 2002, AJ, 123, 3356

Hall, J. C. 1996, PASP, 108, 313

Hartmann, L., Hewett, R., Stahler, S., \& Mathieu, R. D. 1986, ApJ, 309, 275

Hawley, S. L., Gizis, J. E., \& Reid, I. N. 1996, AJ, 112, 2799

Herbig, G. H., \& Bell, K. R. 1988, in Catalog of emission line stars of the orion population, Lick Observatory Bulletin, Santa Cruz, Lick Observatory

Høg, E., Fabricius, C., Makarov, V. V., et al. 2000, A\&A, 355, L27

Holmberg, J., Nordström, B., \& Andersen, J. 2007, A\&A, 475, 519

Holmberg, J., Nordström, B., \& Andersen, J. 2009, A\&A, 501, 941

Hormuth, F., Brandner, W., Hippler, S., et al. 2007, A\&A, 463, 707

Huenemoerder, D. P., \& Barden, S. C. 1984, BAAS, 16, 510

Jensen, E. L. N., Mathieu, R. D., \& Fuller, G. A. 1996, ApJ, 458, 312

Johnson, D. H., \& Soderblom, D. R. 1987, AJ, 93, 864

Klutsch, A., Frasca, A., Guillout, P., et al. 2008, A\&A, 490, 737

López-Santiago, J., Montes, D., Fernández-Figueroa, M. J., \& Ramsey, L. W. 2003, A\&A, 411, 489

López-Santiago, J., Montes, D., Fernández-Figueroa, M. J., et al. 2005, in Proceedings of the 13th Cambridge Workshop on Cool Stars, Stellar Systems and the Sun, 5-9 July, 2004, Hamburg, Germany, ed. F. Favata, G. A. J. Hussain, \& B. Battrick, ESA SP-560, European Space Agency, 775

López-Santiago, J., Montes, D., Crespo-Chacón, I., \& Fernández-Figueroa, M. J. 2006, ApJ, 643, 1160

López-Santiago, J., Micela, G., Sciortino, S., et al. 2007, A\&A, 463, 165

López-Santiago, J., Micela, G., \& Montes, D. 2009, 499, 129

Marcy, G. W., \& Chen, G. H. 1992, ApJ, 390, 550

Martínez-Arnáiz, R., Maldonado, J., Montes, D., Eiroa, C., \& Montesinos, B. 2010, A\&A, in press [arXiv: 1002.4391]

Micela, G., Affer, L., Favata, F., et al. 2007, A\&A, 461, 977

Mihalas, D., \& Binney, J. 1981 (San Francisco: W. H. Freeman and Co.), 422

Montes, D., Fernández-Figueroa, M. J., De Castro, E., et al. 2000, A\&AS, 146, 103

Montes, D., López-Santiago, J., Gálvez, M. C., et al. 2001a, MNRAS, 328, 45

Montes, D., López-Santiago, J., Fernández-Figueroa, M. J., \& Gálvez, M. C. 2001b, A\&A, 379, 976

Neuhaeuser, R., Torres, G., Sterzik, M. F., \& Randich, S. 1997, A\&A, 325, 647

Nidever, D. L., Marcy, G. W., Butler, R. P., et al. 2002, ApJS, 141, 503

Nordström, B., Mayor, M., Andersen, J., et al. 2004, A\&A, 418, 989 
Noyes, R. W., Hartmann, L. W., Baliunas, S. L., et al. 1984, ApJ, 279, 763

Pizzolato, N., Maggio, A., Micela, G., et al. 2003, A\&A, 397, 147

Randich, S., Schmitt, J. H. M. M., Prosser, C. F., \& Stauffer, J. R. 1996, A\&A, 305,785

Rauscher, E., \& Marcy, G. W. 2006, PASP, 118, 617

Reid, I. N., Hawley, S. L., \& Gizis, J. E. 1995, AJ, 110, 1838

Reid, I. N., Gizis, J. E., \& Hawley, S. L. 2002, AJ, 124, 2721

Reiners, A. 2007, in Annual Meeting of the German Astronomical Society [arXiv:0712.2357]

Reiners, A., \& Basri, G. 2007, ApJ, 656, 1121

Rhode, K. L., Herbst, W., \& Mathieu, R. D. 2001, AJ, 122, 3258

Rutten, R. G. M. 1984, A\&A, 130, 353

Rutten, R. G. M., \& Schrijver, C. J. 1987, A\&A, 177, 155

Schmidt-Kaler, T. 1982, in Landolt-Bornstein, ed. K. Schaifers, \& H. H. Voig (Heidelberg: Springer), 2

Sciortino, S., Favata, F., \& Micela, G. 1995, A\&A, 296, 370

Shkolnik, E., Liu, M. C., \& Reid, I. N. 2009, ApJ, 699, 649

Skuljan, J., Hearnshaw, J. B., \& Cottrell, P. L. 1999, MNRAS, 308, 731

Snedecor, G. W., \& Cochran, W. G. 1989, Statistical Methods, eighth edn. (Ames, Iowa: Iowa State University Press)

Soderblom, D. R. 1982, ApJ, 263, 239

Soderblom, D. R., \& Mayor, M. 1993, ApJ, 402, L5

Soderblom, D. R., Pendleton, J., \& Pallavicini, R. 1989, AJ, 97, 539

Soderblom, D. R., Jones, B. F., Balachandran, S., et al. 1993, AJ, 106, 1059

Skumanich, A. 1972, ApJ, 171, 565

Strassmeier, K. G., \& Fekel, F. C. 1990, A\&A, 230, 389

Strassmeier, K. G., Hall, D. S., Fekel, F. C., \& Scheck, M. 1993, A\&AS, 100, 173

Strassmeier, K. G., Washuettl, A., Granzer, Th., et al. 2000, A\&AS, 142, 275

Takeda, G., Ford, E. B., Sills, A., et al. 2007, ApJS, 168, 297

Tokovinin, A. A. 1992, A\&A, 256, 121

Tonry, J., \& Davis, M. 1979, AJ, 84, 1511

Torres, C. A. O., Quast, G. R., da Silva, L., et al. 2006, A\&A, 460, 695

Torres, C. A. O., Quast, G. R., Melo, C. H. F., \& Sterzik, M. F. 2008, Handbook of Star Forming Regions, Volume II: The Southern Sky, ASP Monograph Publications, ed. B. Reipurth, 5, 757

Toth, G., \& Ostriker, J. P. 1992, ApJ, 389, 5

Udry, S., Mayor, M., Maurice, E., et al. 1999, in Precise Stellar Radial Velocities, ed. J. B. Hearnshaw, \& C. D. Scarfe, IAU Colloq., 170, ASP Conf. Ser., 185, 383

Valenti, J. A., \& Fischer, D. A. 2005, ApJS, 159, 141

Vaughan, A. H., \& Preston, G. W. 1980, PASP, 92, 385

Walkowicz, L. M., Hawley, S. L., \& West, A. A. 2004, PASP, 116, 1105

Walkowicz, L. M., \& Hawley, S. L. 2009, AJ, 137, 3297

West, A. A., Hawley, S. L., Walkowicz, L. M., et al. 2004, AJ, 128, 426

West, A. A., Hawley, S. L., Bochanski, J. J., et al. 2008, AJ, 135, 785

West, A. A., Hawley, S. L., Bochanski, J. J., et al. 2009, IAU Symp., 258, 327

White, R. J., Gabor, J. M., \& Hillenbrand, L. A. 2007, AJ, 133, 2524

Zuckerman, B., Song, I., \& Bessell, M. S. 2004, ApJ, 613, L65

\section{Appendix A: Description of the on-line material}

- Table A.1: spectral-type reference stars and radial velocity standards used for the subtraction of the photospheric spectrum and for determining radial and rotational velocities. Column 1 is the name/identification of the star; Col. 2 is the spectral type; Col. 3 is the radial velocity (and standard deviation); Col. 4 is the bibliographic reference for the radial velocity; Col. 5 is the rotational velocity (and standard deviation); Col. 6 is the bibliographic reference of the rotational velocity; Col. 7 is the activity index $\mathrm{S}$ (and standard deviation); and Col. 8 is the observing run in which the star was observed by us.

- Table A.2: spectroscopic parameters of the stars in the sample. Each line corresponds to a measurement/observation of the star. Column 1 is the HD number or other name of the star; Col. 2 is the observing run in which the observation was performed; Col. 3 is the Modified Julian Date of the observation; Col. 4 is the spectral type of the star determined by us; Col. 5 is the $B-V$ color from Tycho-2; Col. 6 is the radial velocity determined by us in that observation; Col. 7 is a mean radial velocity of every observation performed for the star; Cols. 8-10 are the Galactic velocities; Col. 11 is the rotational velocity determined in that observation; Col. 12 is a mean value of the rotational velocity of the star determined in each observation; Col. 13 is the photometric period found in the literature; Col. 14 is the equivalent width of the lithium line in $6707.8 \AA$ determined in that observation; Col. 15 is a mean value of the equivalent width of the lithium line of the star; and Col. 16 is the preliminary assignation to a moving group made by us in base to the Galactic velocity given in Cols. 8-10.

- Table A.3: equivalent widths of the different chromospheric lines determined in each observation from the subtracted spectrum. Column 1 is the identification of the star in our sample (the same as in Table A.2; Col. 2 is the observing run of the observation; Col. 3 is the Modified Julian Date of the observation; Cols. 4 to 16 are the equivalent widths in the chromospheric lines (in order): $\mathrm{Ca}$ II $\mathrm{K} \& \mathrm{H}, \mathrm{H} \epsilon, \mathrm{H} \delta$, $\mathrm{H} \gamma, \mathrm{H} \beta, \mathrm{He} \mathrm{I} \mathrm{D}_{3}, \mathrm{Na} \mathrm{I} \mathrm{D}_{2} \& \mathrm{D}_{1}, \mathrm{H} \alpha$, and Ca II infrared triplet ( $\lambda \lambda$ 8498, 8542, $8662 \AA)$, with errors.

- Table A.4: surface fluxes of the different chromospheric lines determined in each observation from the equivalent widths in Table A.3. Column 1 is the identification of the star in our sample (the same as in Table A.2; Col. 2 is the observing run of the observation; Col. 3 is the Modified Julian Date of the observation; Cols. 4 to 16 are the surface fluxes in the chromospheric lines (in order): Ca II $\mathrm{K} \& \mathrm{H}, \mathrm{H} \epsilon, \mathrm{H} \delta, \mathrm{H} \gamma, \mathrm{H} \beta$, $\mathrm{He}_{\mathrm{I}} \mathrm{D}_{3}, \mathrm{Na}$ I $\mathrm{D}_{2} \& \mathrm{D}_{1}, \mathrm{H} \alpha$, and $\mathrm{Ca}$ II infrared triplet $(\lambda \lambda 8498$, $8542,8662 \AA$ ), with errors; Col. 17 is the $R_{\mathrm{HK}}^{\prime}$ index.

- Figures A.1 to A.4: figures with the normalized spectrum of the stars in our sample in the spectral regions of the Ca II K, $\mathrm{H} \alpha$, Li I, and Ca II $\lambda \lambda 8498$ and $8542 \AA$ lines, respectively. 
A\&A 514, A97 (2010)

Table A.1. Spectral-type reference stars and radial velocity standards (marked with *).

\begin{tabular}{|c|c|c|c|c|c|c|c|}
\hline HD/GJ & SpT & $\begin{array}{l}V_{\mathrm{r}} \pm \sigma_{V_{\mathrm{r}}} \\
\left(\mathrm{km} \mathrm{s}^{-1}\right)\end{array}$ & $\operatorname{Ref}_{\mathrm{v}}$ & $\begin{array}{c}v \sin i \\
\left(\mathrm{~km} \mathrm{~s}^{-1}\right)\end{array}$ & $\operatorname{Ref}_{\mathrm{r}}$ & $S \pm \sigma_{\mathrm{S}}$ & Observing run \\
\hline 212754 & F7 V & $-17.8 \pm 1.2$ & $\mathrm{a}$ & $7.9 \pm 0.7$ & $\dot{j}$ & $0.142 \pm 0.001$ & 4,5 \\
\hline $43587 *$ & F9 V & $4.6 \pm 0.1$ & $\mathrm{~b}$ & $\ldots$ & & $0.158 \pm 0.001$ & 5,6 \\
\hline $84737 *$ & G0.5 V & $6.0 \pm 1.1$ & $\mathrm{~b}$ & $2.8 \pm 0.8$ & $j$ & $0.144 \pm 0.000$ & 10 \\
\hline 10307 & $\mathrm{G} 2 \mathrm{~V}$ & $\ldots$ & & $2.1 \pm 0.5$ & $\mathrm{k}$ & $0.152 \pm 0.003$ & 7 \\
\hline 193664 & G3 V & $-4.7 \pm 1.2$ & $\mathrm{a}$ & $\ldots$ & & $0.161 \pm 0.004$ & 7 \\
\hline 25680 & G5 V & $24.0 \pm 0.1$ & $\mathrm{c}$ & $7.0 \pm 0.7$ & $\mathrm{j}$ & $0.281 \pm 0.000$ & 5,7 \\
\hline 31966 & G5 V & $-18.1 \pm 0.1$ & $\mathrm{c}$ & .. & & $\ldots$ & 5 \\
\hline $71148 *$ & G5 V & $-31.0 \pm 0.7$ & $\mathrm{~b}$ & $\ldots$ & & $1.570 \pm 0.000$ & 10 \\
\hline $159222 *$ & G5 V & $-50.5 \pm 1.2$ & $\mathrm{~b}$ & $\ldots$ & & $0.164 \pm 0.002$ & $1,4,11$ \\
\hline $182488 *$ & G8 V & -21.5 & $\mathrm{~d}$ & $0.6 \pm 0.5$ & $\mathrm{k}$ & $0.155 \pm 0.008$ & $1,4,11$ \\
\hline 48432 & K0 III & $17.9 \pm 0.2$ & $\mathrm{e}$ & $<1.0$ & $\mathrm{e}$ & $0.120 \pm 0.000$ & 5,6 \\
\hline $62509 *$ & K0 III & $3.2 \pm 0.3$ & $\mathrm{~b}$ & $1.7 \pm 0.5$ & $\mathrm{k}$ & $0.140 \pm 0.019$ & 3 \\
\hline $100696 *$ & K0 III & $0.2 \pm 0.5$ & $\mathrm{~b}$ & $1.2 \pm 1.0$ & 1 & $\ldots$ & 10 \\
\hline 197989 & K0 III & $-10.6 \pm 0.5$ & $\mathrm{a}$ & $2.0 \pm 0.5$ & $\mathrm{k}$ & $0.104 \pm 0.001$ & $1,2,4,7,8,11$ \\
\hline $3651 *$ & $\mathrm{~K} 0 \mathrm{~V}$ & $-32.8 \pm 0.8$ & $\mathrm{~b}$ & $2.2 \pm 0.5$ & $\mathrm{k}$ & $0.191 \pm 0.001$ & $2,3,4,5,7$ \\
\hline 97004 & $\mathrm{~K} 0 \mathrm{~V}$ & $5.4 \pm 0.1$ & $\mathrm{c}$ & $\ldots$ & & $\ldots$ & 10 \\
\hline 112758 & $\mathrm{~K} 0 \mathrm{~V}$ & $-4.1 \pm 1.2$ & a & $\ldots$ & & $0.206 \pm 0.000$ & 10 \\
\hline $136442 *$ & K0 V & $-45.6 \pm 0.8$ & $\mathrm{~b}$ & $\ldots$ & & $\ldots$ & 10 \\
\hline 185144 & $\mathrm{~K} 0 \mathrm{~V}$ & $26.7 \pm 0.1$ & $\mathrm{c}$ & $0.6 \pm 0.5$ & $\mathrm{k}$ & $0.195 \pm 0.003$ & 11 \\
\hline 201651 & K0 V & $-13.7 \pm 1.2$ & $\mathrm{a}$ & $\ldots$ & & $\ldots$ & $4,7,8$ \\
\hline $92588 *$ & K1 IV & $43.5 \pm 0.3$ & $\mathrm{f}$ & $<1.0$ & e & $\ldots$ & 9,10 \\
\hline $10476 *$ & $\mathrm{~K} 1 \mathrm{~V}$ & $-33.9 \pm 0.9$ & $\mathrm{~b}$ & $0.6 \pm 0.5$ & $\mathrm{k}$ & $0.192 \pm 0.001$ & 1,5 \\
\hline $12929 *$ & K2 III & $-14.6 \pm 0.2$ & g & $1.8 \pm 0.5$ & $\mathrm{k}$ & $0.118 \pm 0.002$ & 5 \\
\hline $124897 *$ & K2 III & $-5.3 \pm 0.3$ & $\mathrm{~g}$ & $3.3 \pm 0.5$ & $\mathrm{k}$ & $0.144 \pm 0.012$ & 6,10 \\
\hline $161096 *$ & K2 III & $-12.5 \pm 0.3$ & $\mathrm{~g}$ & $2.5 \pm 0.5$ & $\mathrm{k}$ & $0.103 \pm 0.002$ & $1,4,11$ \\
\hline 201196 & K2 IV & $-34.8 \pm 0.2$ & $\mathrm{e}$ & $<1.0$ & $\mathrm{e}$ & $\ldots$ & 1,5 \\
\hline $4628 *$ & $\mathrm{~K} 2 \mathrm{~V}$ & $-10.1 \pm 0.4$ & $\mathrm{f}$ & $0.0 \pm 0.5$ & $\mathrm{~h}$ & $0.223 \pm 0.001$ & $2,5,8,12$ \\
\hline 136713 & K2 V & $-6.0 \pm 0.1$ & $\mathrm{c}$ & $3.8 \pm 5.7$ & $\mathrm{~m}$ & $\ldots$ & 6 \\
\hline 166620 & $\mathrm{~K} 2 \mathrm{~V}$ & $6.9 \pm 0.1$ & $\mathrm{~h}$ & $0.0 \pm 0.4$ & $\mathrm{~h}$ & $0.193 \pm 0.001$ & $1,2,4,6,7,9,10,11$ \\
\hline 16160 & K3 V & $25.8 \pm 0.1$ & $\mathrm{c}$ & $1.0 \pm 1.0$ & $\mathrm{~h}$ & $0.221 \pm 0.002$ & 8 \\
\hline 219134 & $\mathrm{~K} 3 / 4 \mathrm{~V}$ & $-18.6 \pm 0.1$ & $\mathrm{c}$ & $2.1 \pm 0.5$ & $\mathrm{k}$ & $0.229 \pm 0.003$ & $1,2,4,5,11$ \\
\hline $29139 *$ & K5 III & $54.2 \pm 0.2$ & $\mathrm{~g}$ & $2.0 \pm 1.0$ & $\mathrm{e}$ & $\ldots$ & $4,6,7$ \\
\hline 154363 & K5 V & $34.1 \pm 0.1$ & $\mathrm{c}$ & $3.7 \pm 5.9$ & & $0.197 \pm 0.001$ & $1,6,10$ \\
\hline 201091 & K5 V & $7.0 \pm 0.1$ & $\mathrm{~h}$ & $0.0 \pm 0.8$ & $\mathrm{~h}$ & $0.613 \pm 0.006$ & $1,4,5,6,7,8,10,11$ \\
\hline GJ 910 & K5 V & 2.0 & $\mathrm{i}$ & $0.0 \pm 0.0$ & $\mathrm{~m}$ & $\ldots$ & 7 \\
\hline 151877 & K7 V & $2.0 \pm 0.1$ & $\mathrm{c}$ & $0.0 \pm 0.0$ & $\mathrm{~m}$ & $\ldots$ & 1 \\
\hline 201092 & $\mathrm{~K} 7 \mathrm{~V}$ & $7.2 \pm 0.1$ & $\mathrm{~h}$ & $1.7 \pm 0.6$ & $\mathrm{~h}$ & $0.922 \pm 0.011$ & $1,4,5,6,7,8,10,11$ \\
\hline GJ 466 & M0 V & $-5.0 \pm 5.0$ & $\mathrm{a}$ & $\ldots$ & & $\ldots$ & 10 \\
\hline 147379 & M0 V & $-18.8 \pm 0.1$ & $\mathrm{c}$ & $4.2 \pm 6.2$ & $\mathrm{~m}$ & $1.761 \pm 0.160$ & 11 \\
\hline GJ 720A & M0 V & $-25.0 \pm 2.5$ & $\mathrm{a}$ & $6.3 \pm 1.7$ & $\mathrm{~m}$ & $\ldots$ & 7 \\
\hline GJ 16 & $\mathrm{M} 0 / 1 \mathrm{~V}$ & $\ldots$ & & $\ldots$ & & $\ldots$ & 8 \\
\hline GJ 806 & M1.5 V & $-24.7 \pm 0.1$ & $\mathrm{c}$ & $1.9 \pm 0.7$ & $\mathrm{n}$ & $\ldots$ & 11 \\
\hline $18884 *$ & M2 III & $-26.1 \pm 0.3$ & $\mathrm{~g}$ & $\ldots$ & & $0.331 \pm 0.004$ & 8 \\
\hline $115521 *$ & M2 III & $-28.6 \pm 2.3$ & $\mathrm{~g}$ & $\ldots$ & & $\ldots$ & 6,10 \\
\hline 95735 & $\mathrm{M} 2 \mathrm{~V}$ & $-84.7 \pm 0.1$ & c & $0.0 \pm 0.0$ & $\mathrm{n}$ & $0.392 \pm 0.009$ & 6,10 \\
\hline GJ $687 B^{\dagger}$ & M3.5 V & $-28.8 \pm 0.1$ & $\mathrm{c}$ & $\ldots$ & & $\ldots$ & 6,10 \\
\hline
\end{tabular}

Notes. ${ }^{(\dagger)}$ The radial velocity given for GJ 687B is that of GJ 687A.

References. a Dufflot et al. (1995), WEB (Wilson Evans Batten Catalogue); b Barnes et al. (1986); c Nidever et al. (2002); d ELODIE; e De Medeiros \& Major (1999); f Beavers et al. (1979); g Udry et al. (1999); h Benz \& Major (1984); i Dyer (1954); j Soderblom (1982), Soderblom et al. (1989); k Fekel (1997); 1 De Medeiros et al. (2000); m Tokovinin (1992); n Marcy \& Chen (1992). 


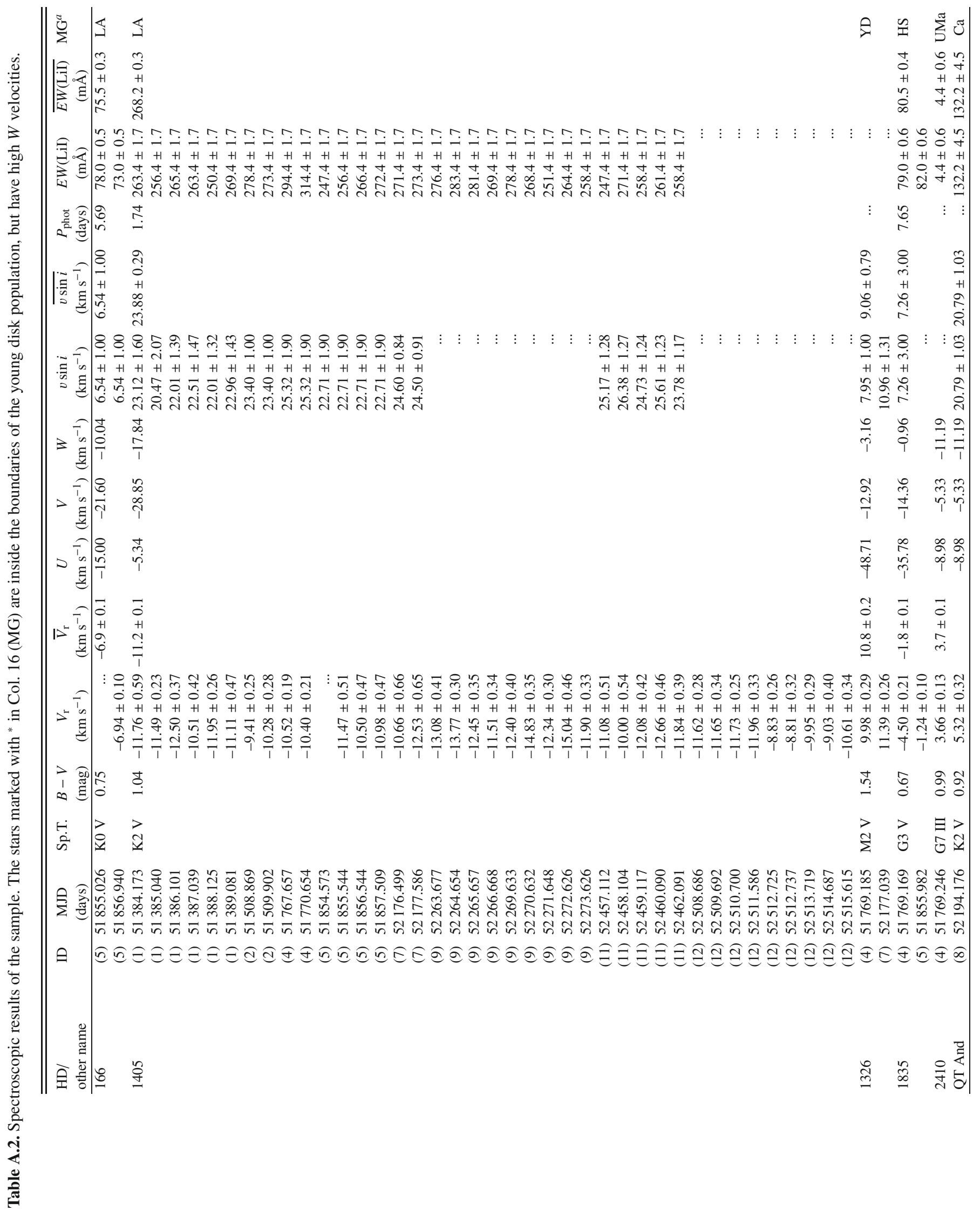




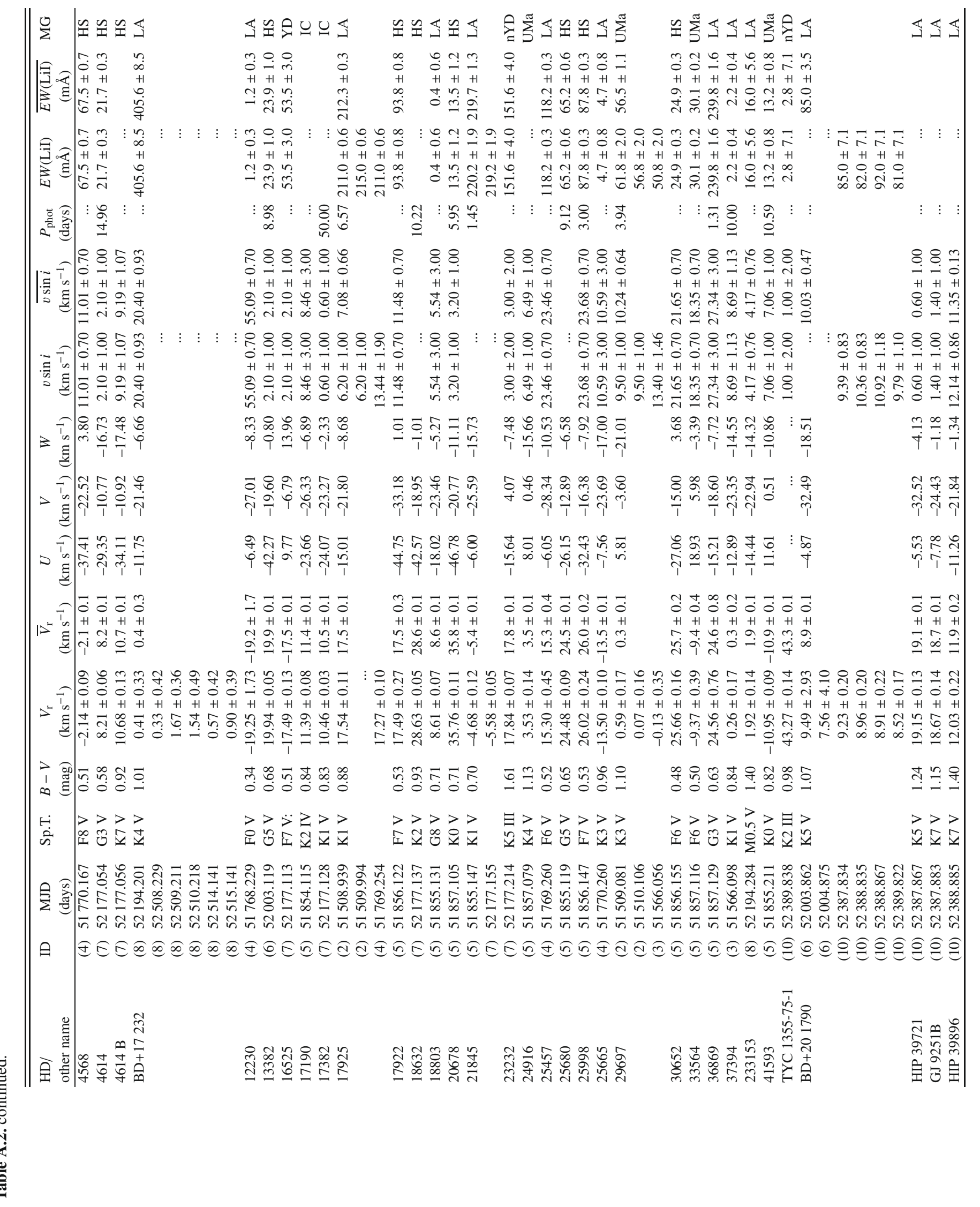

Page 12 of 58 


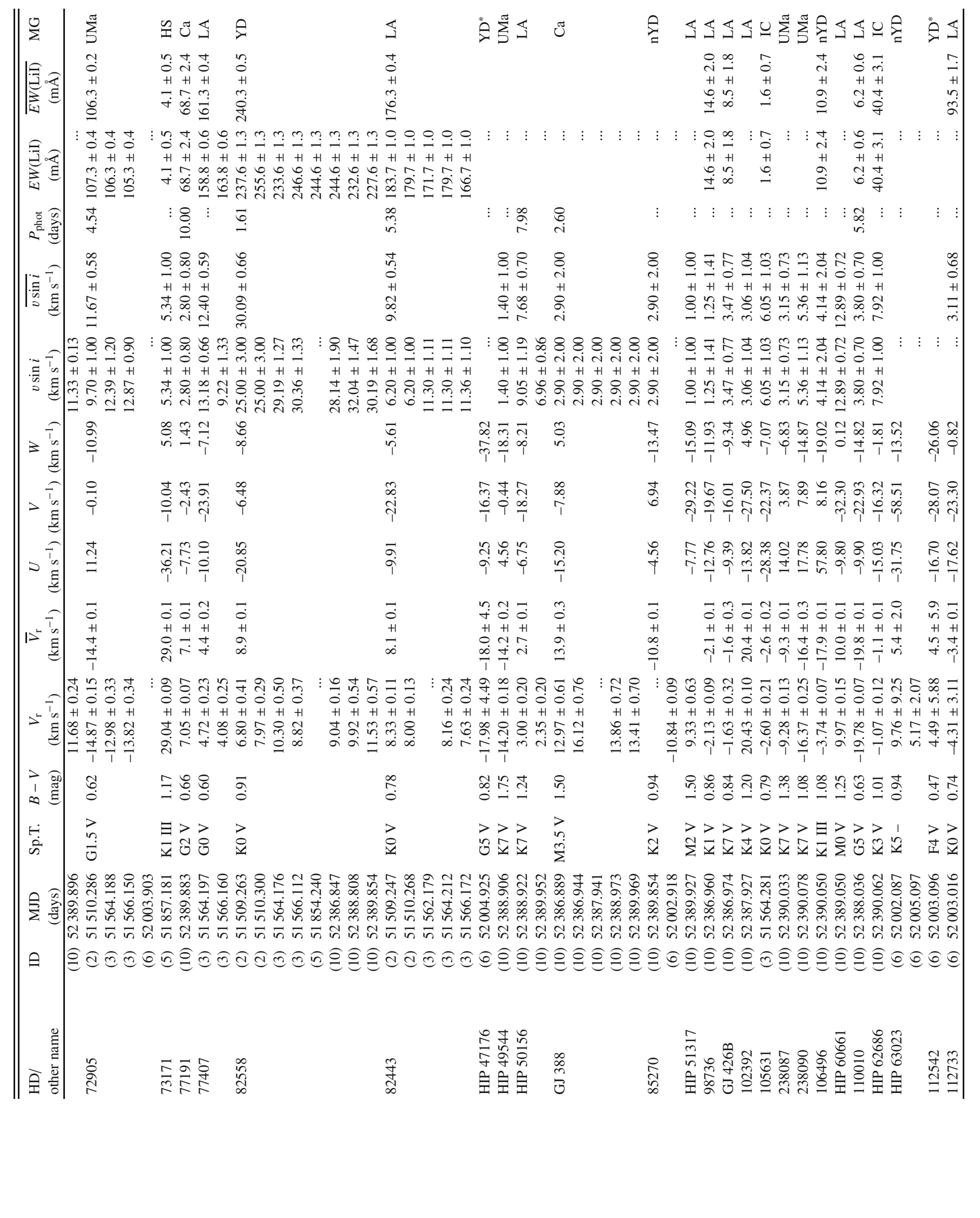




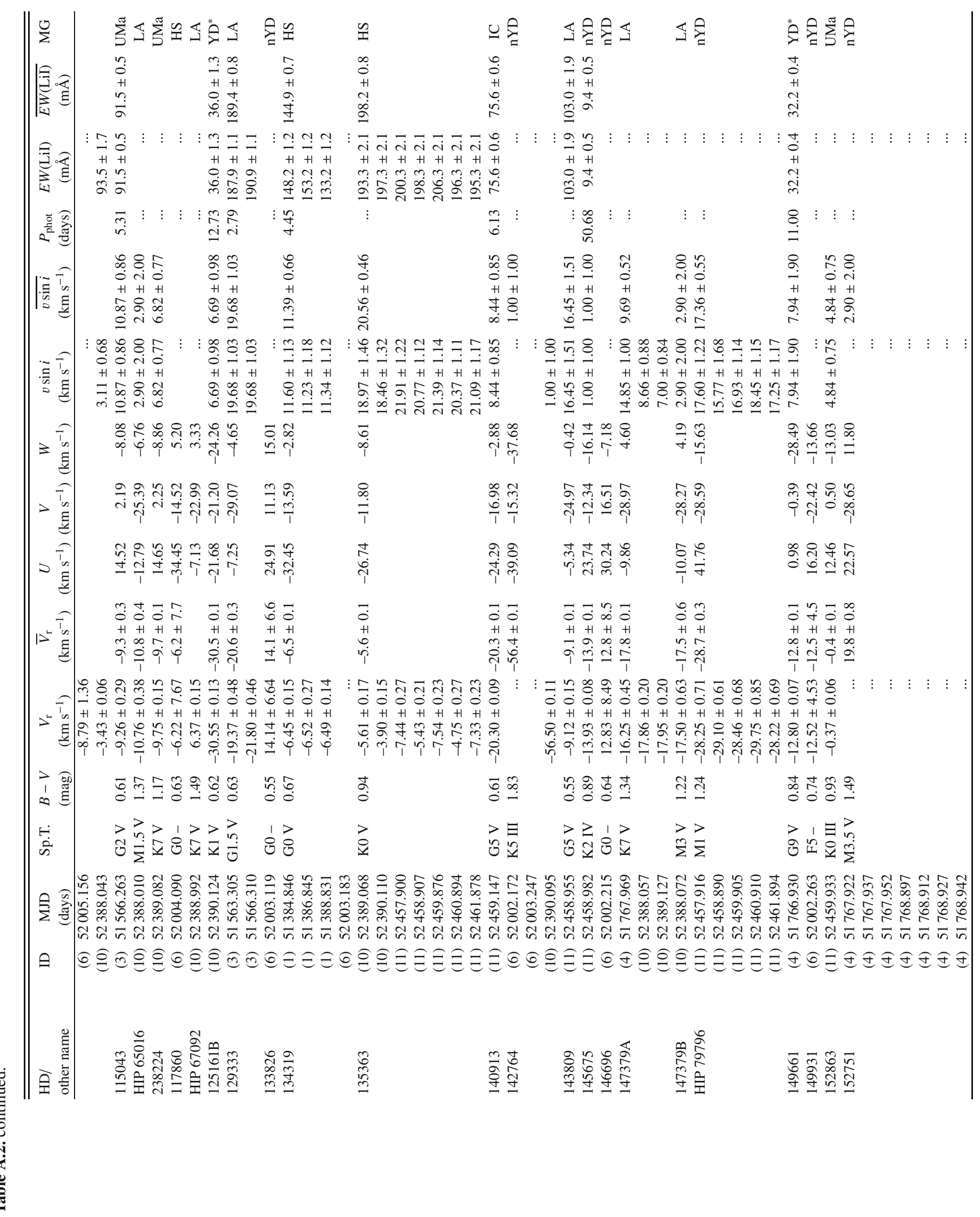




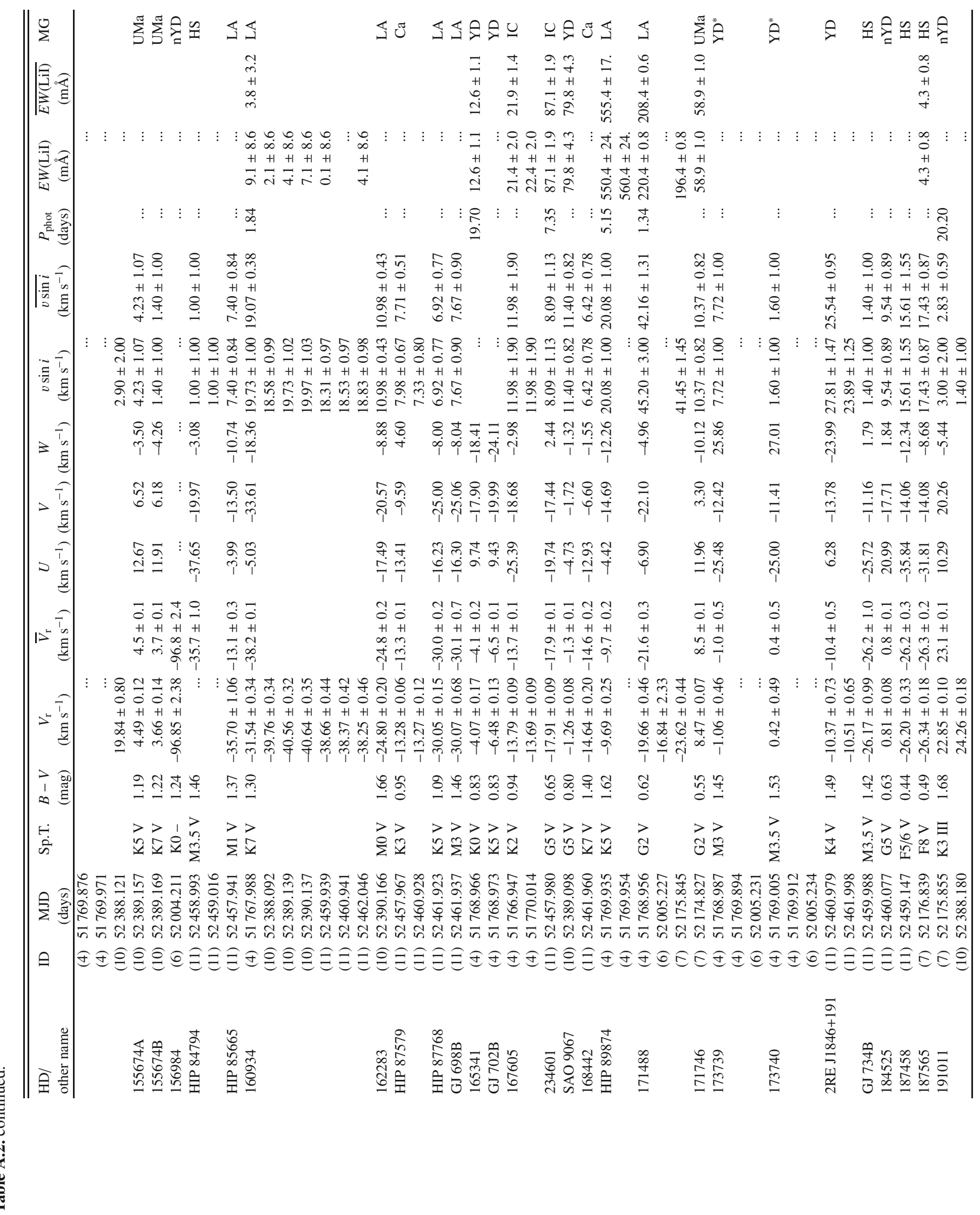




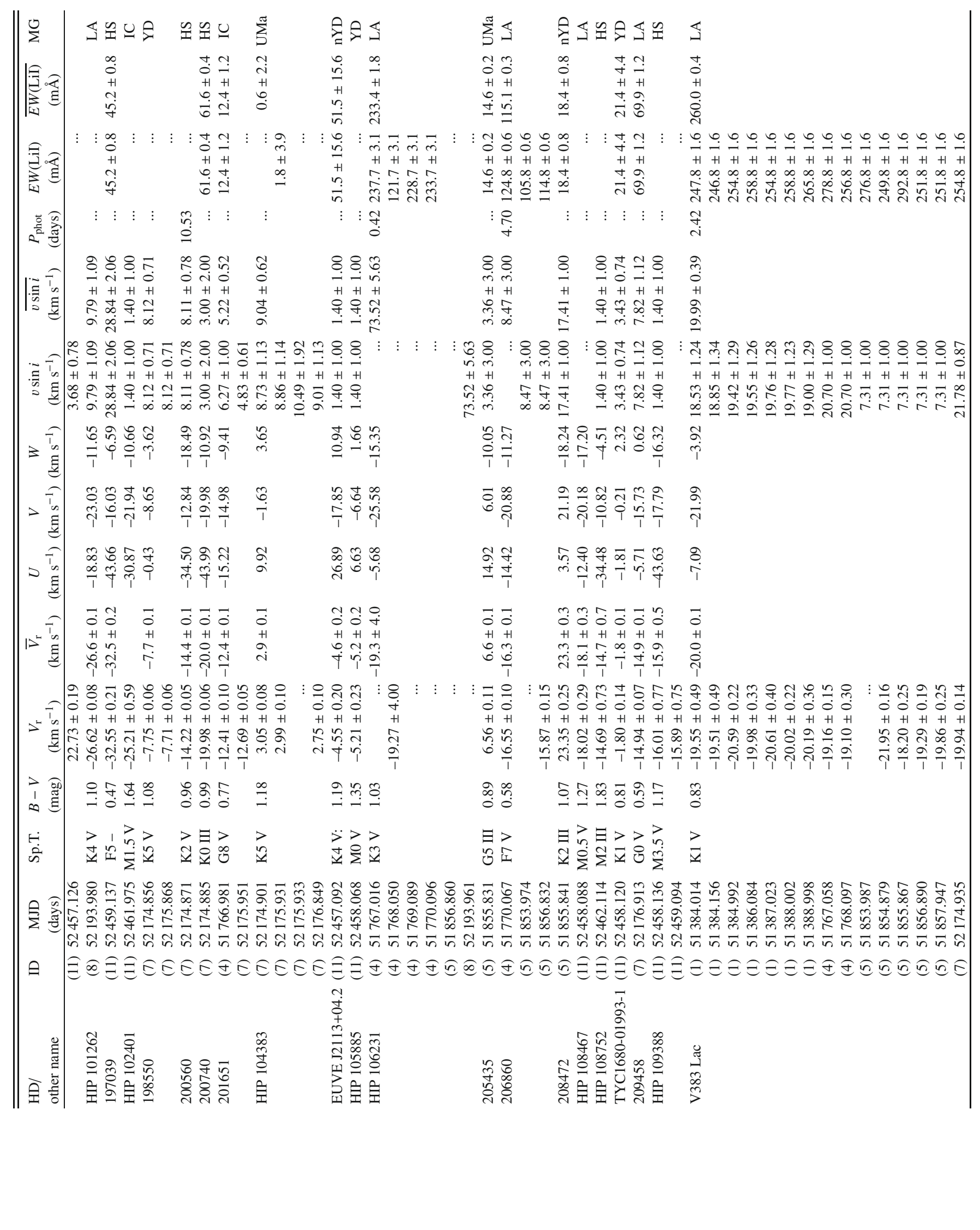




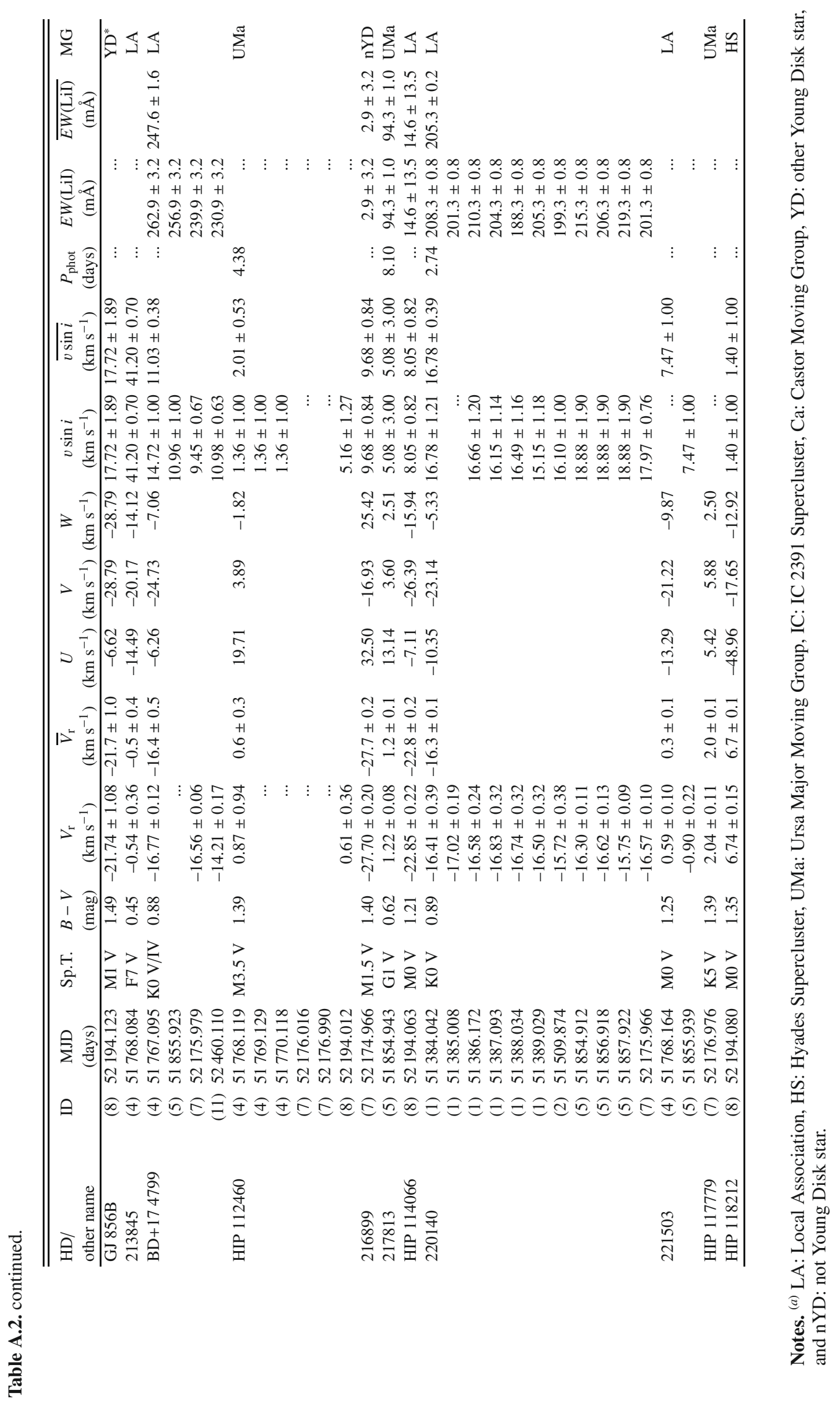




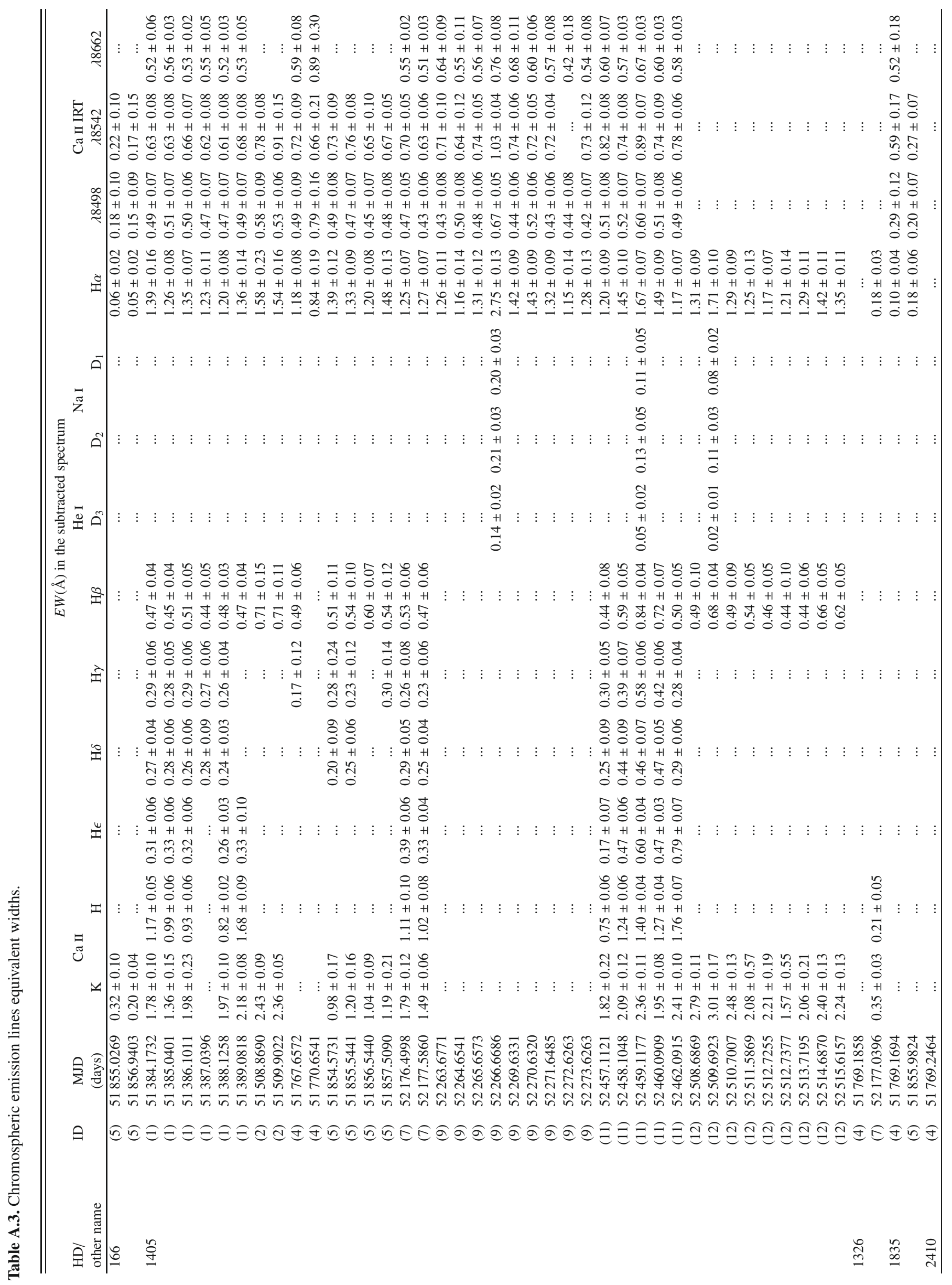

Page 18 of 58 
J. López-Santiago et al.: High-resolution spectroscopic survey of late-type stars

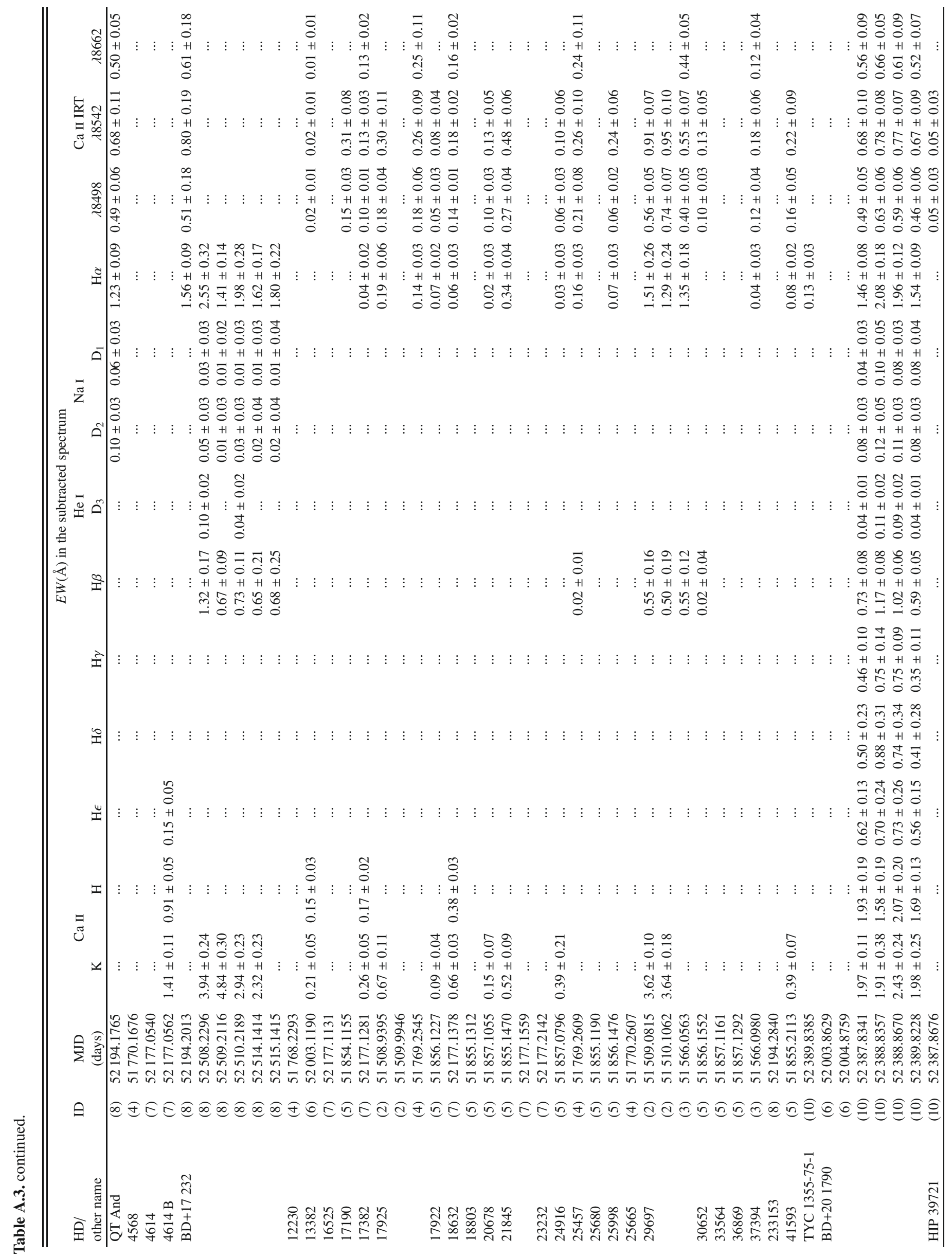




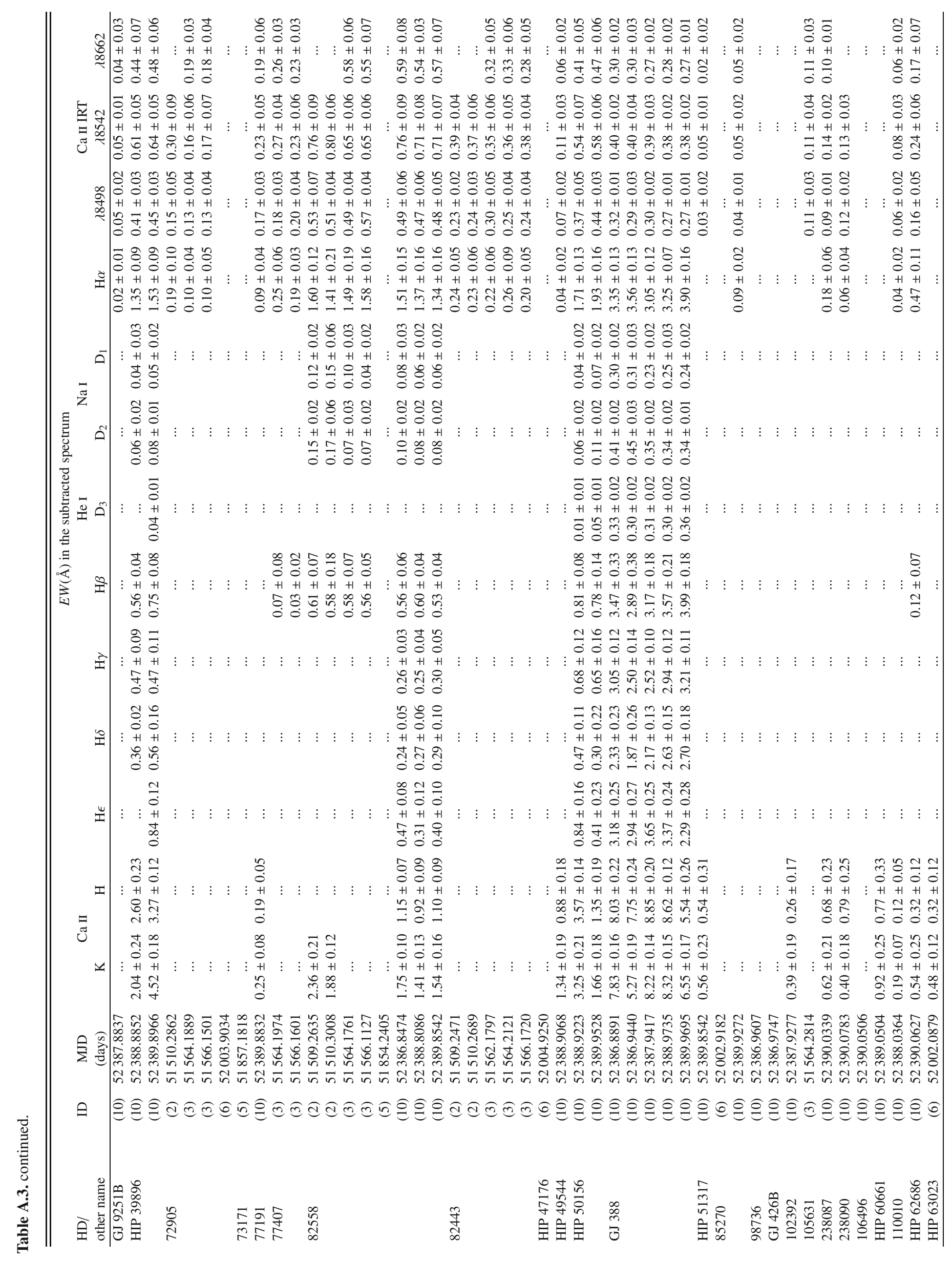




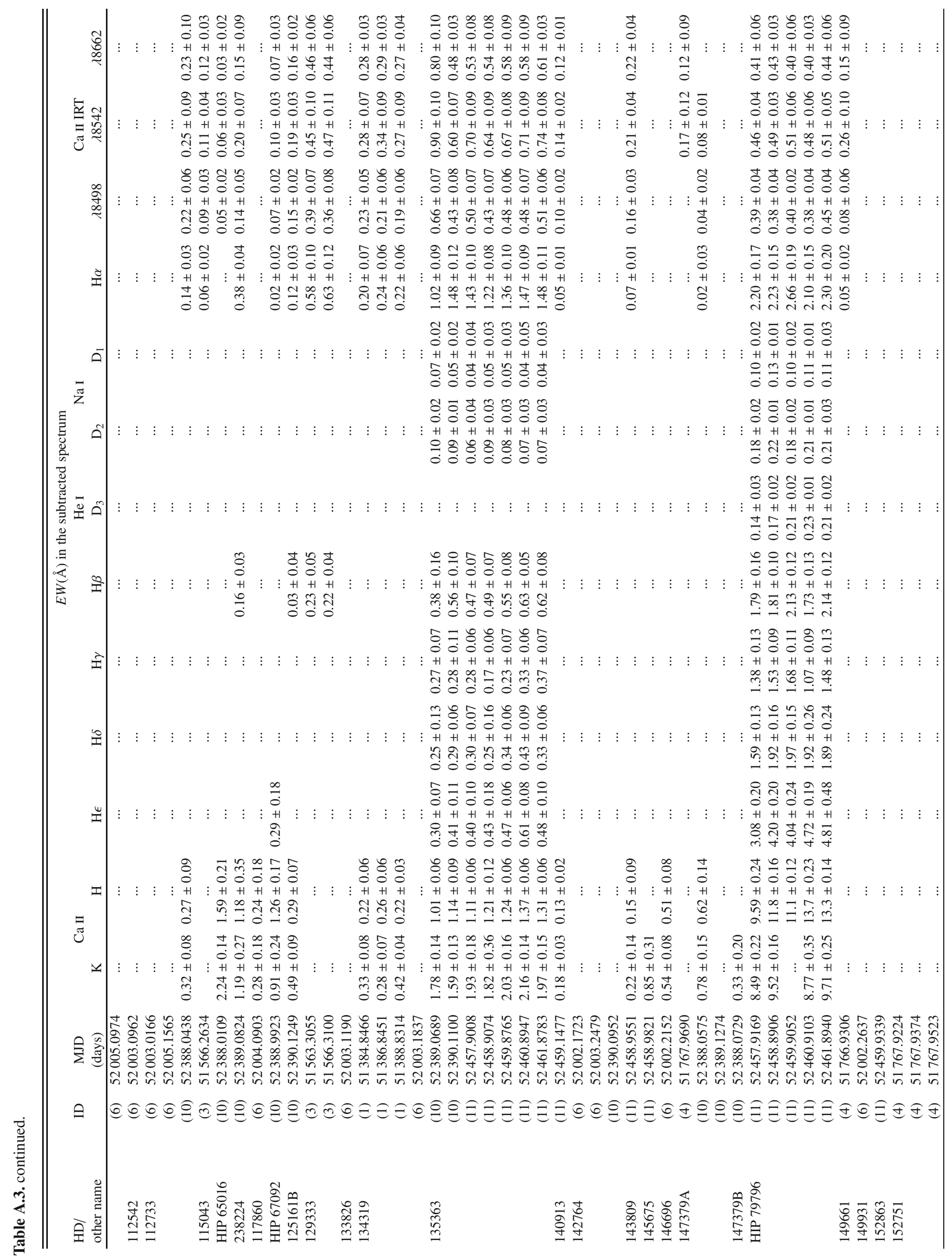




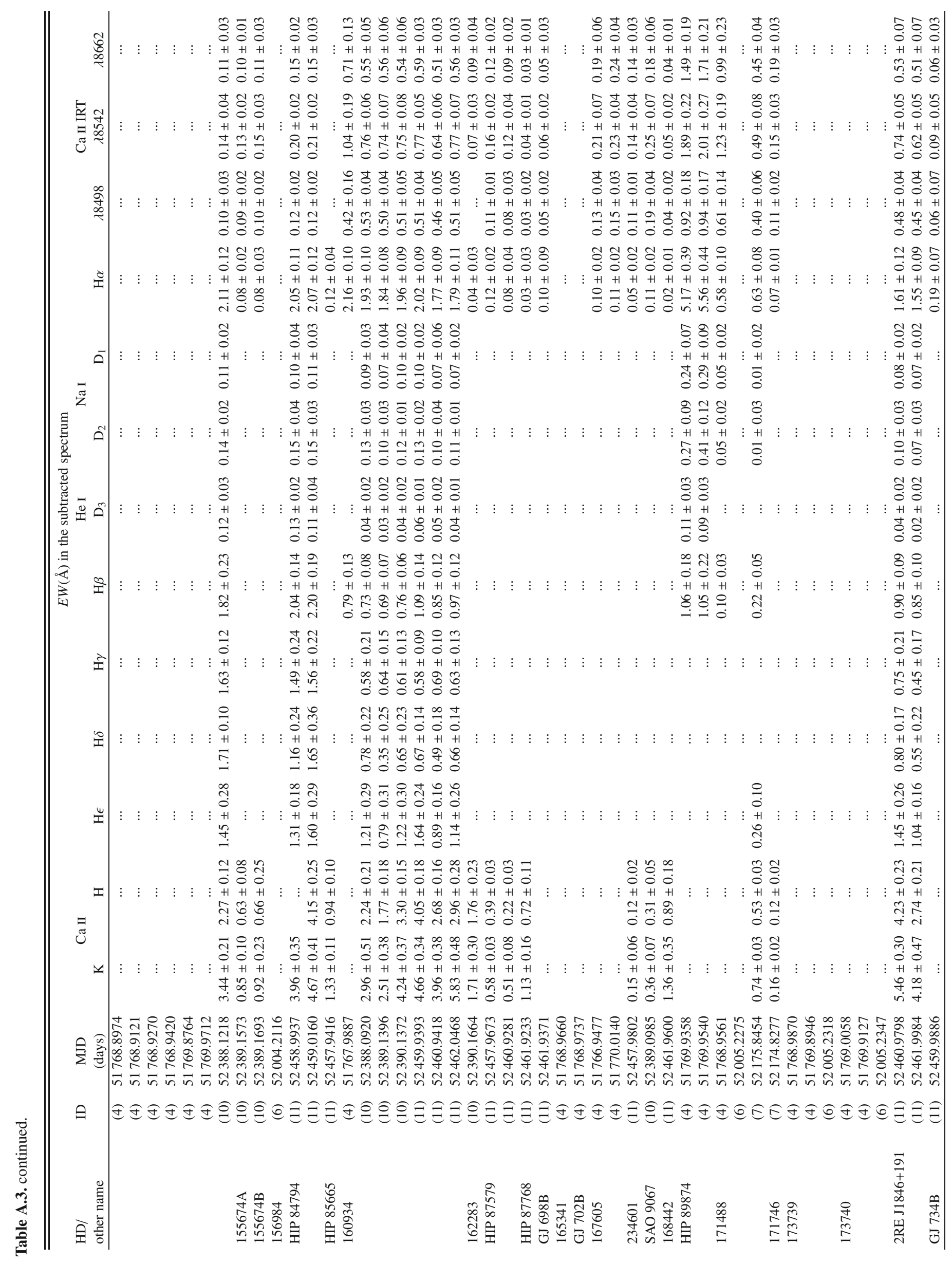




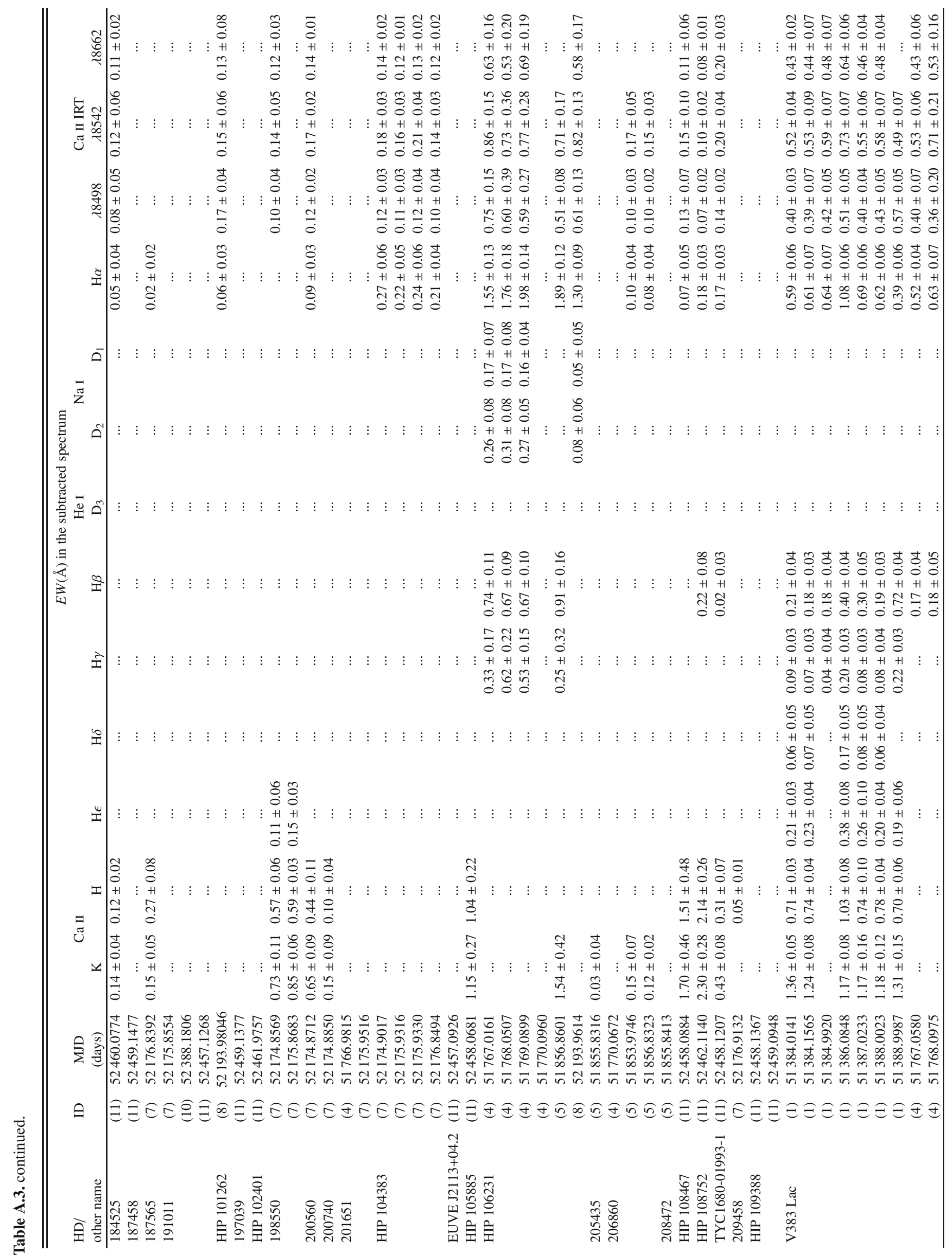




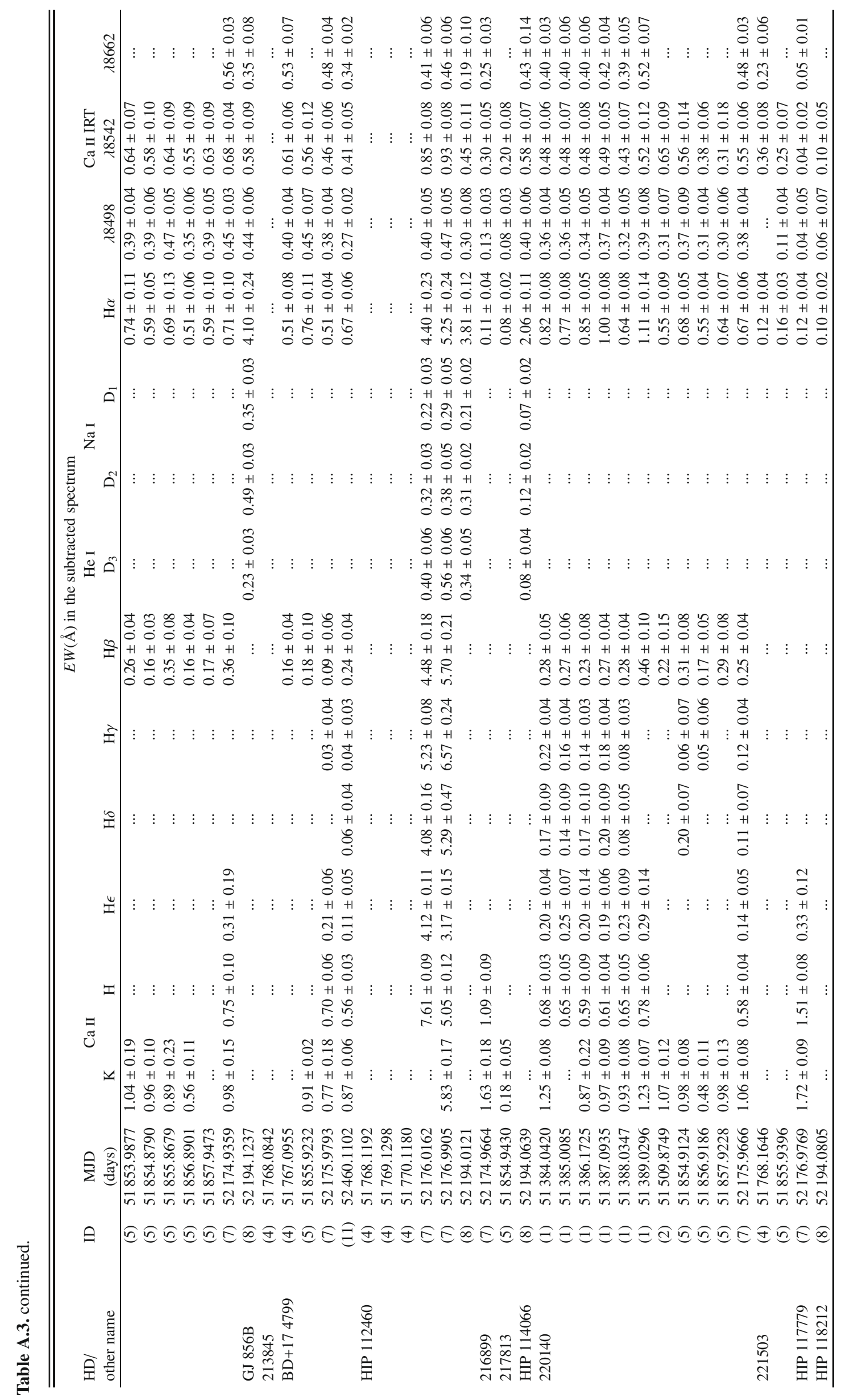


J. López-Santiago et al.: High-resolution spectroscopic survey of late-type stars

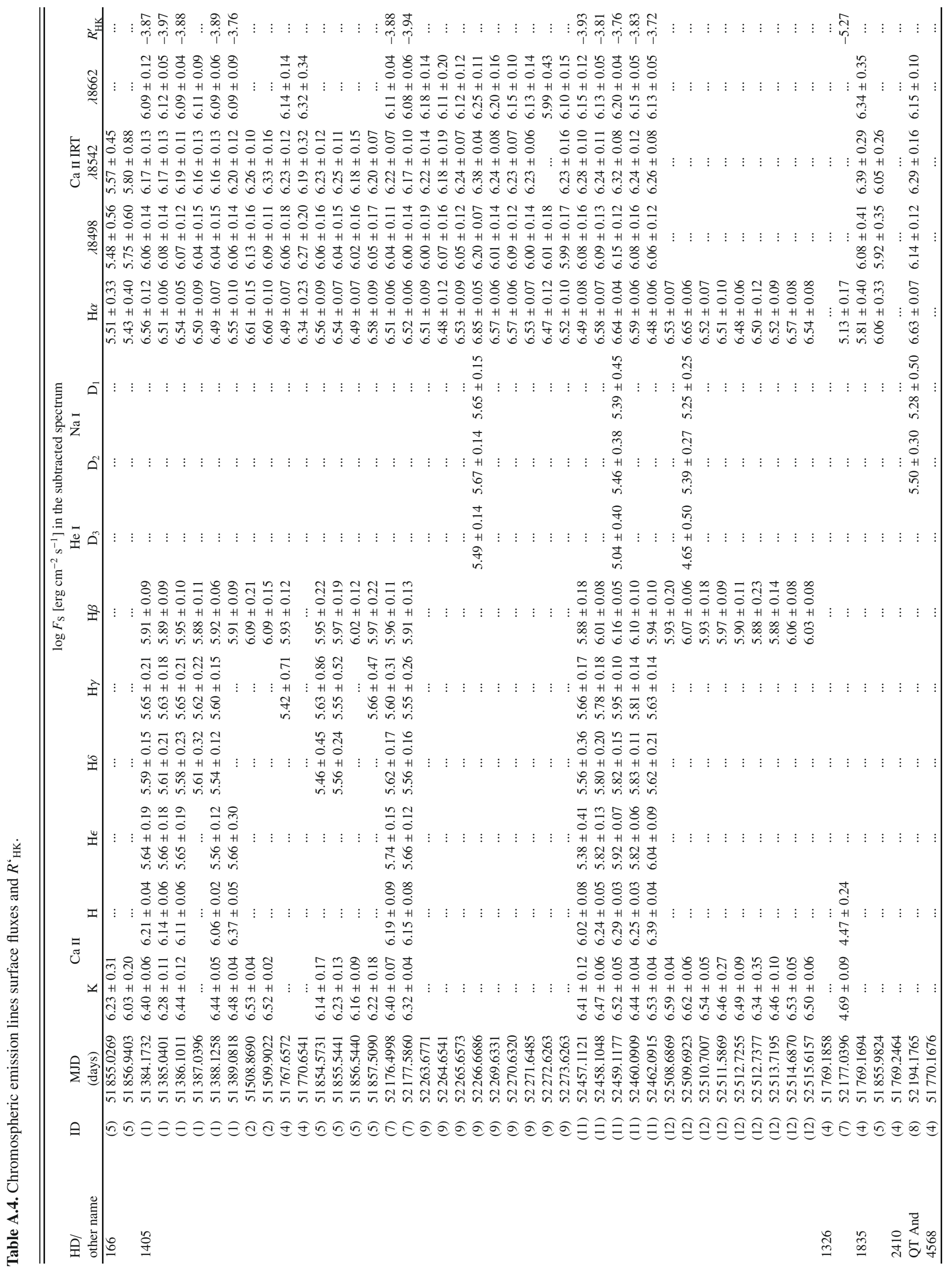

Page 25 of 58 


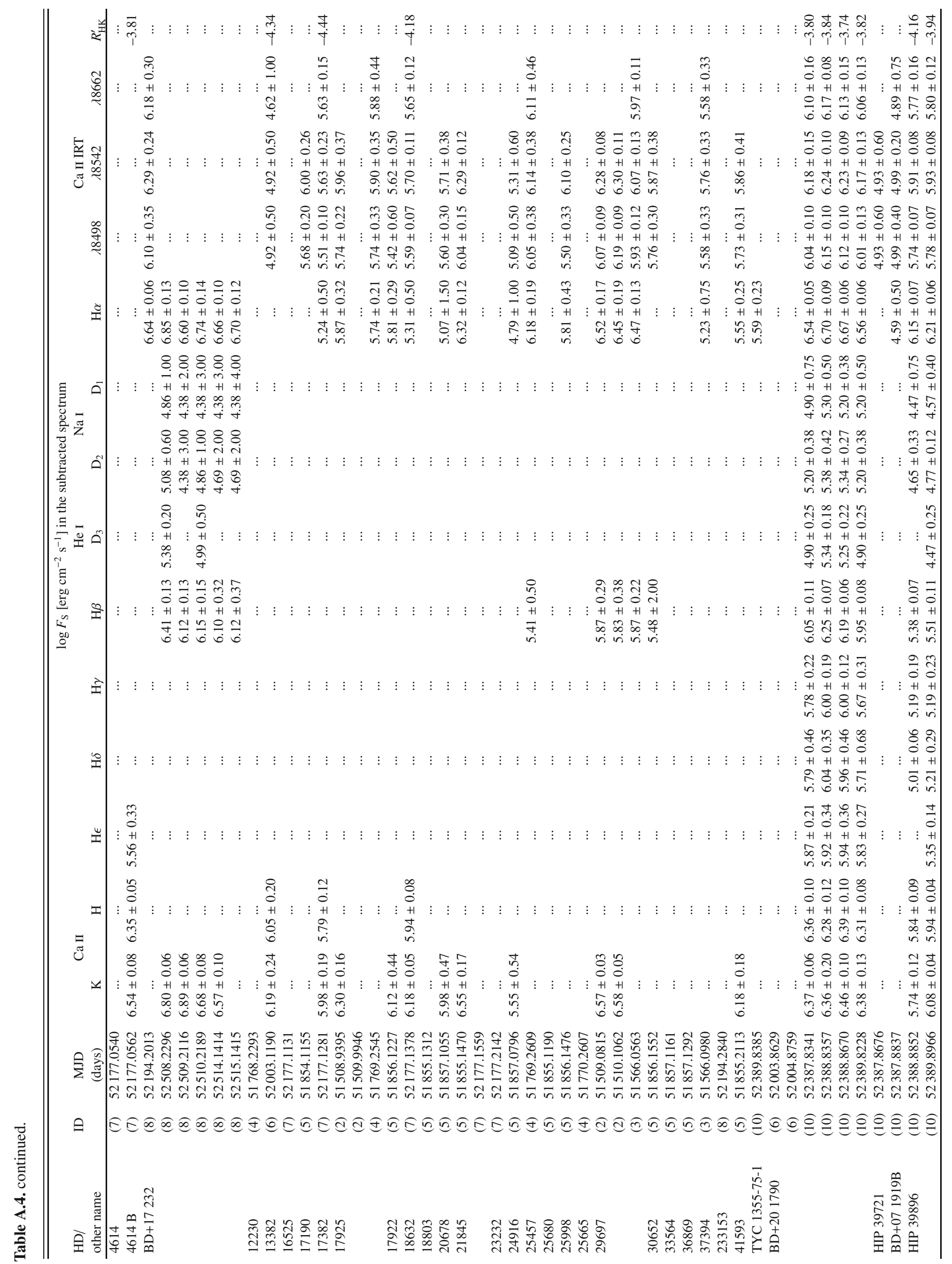

Page 26 of 58 
J. López-Santiago et al.: High-resolution spectroscopic survey of late-type stars

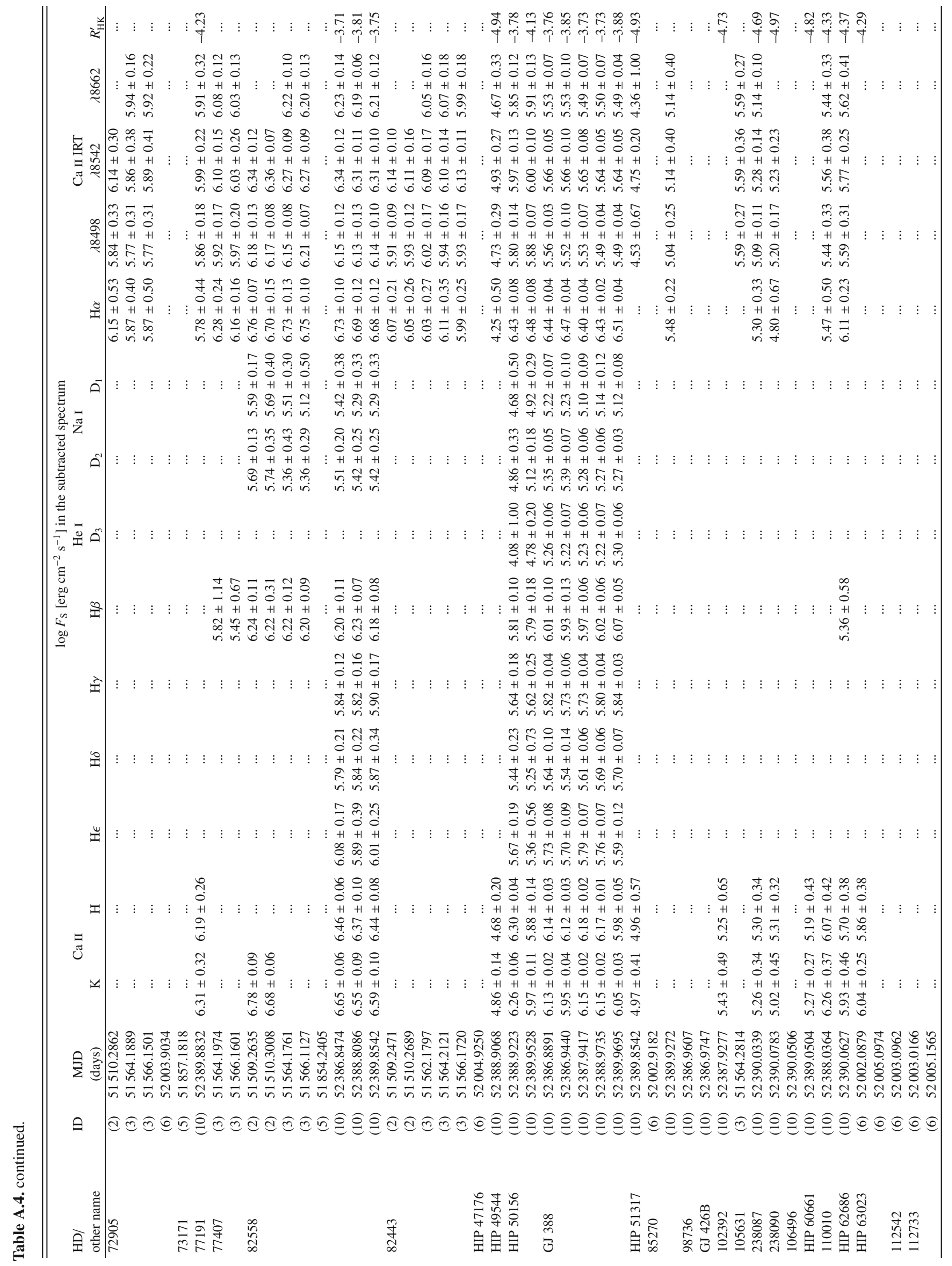


A\&A 514, A97 (2010)

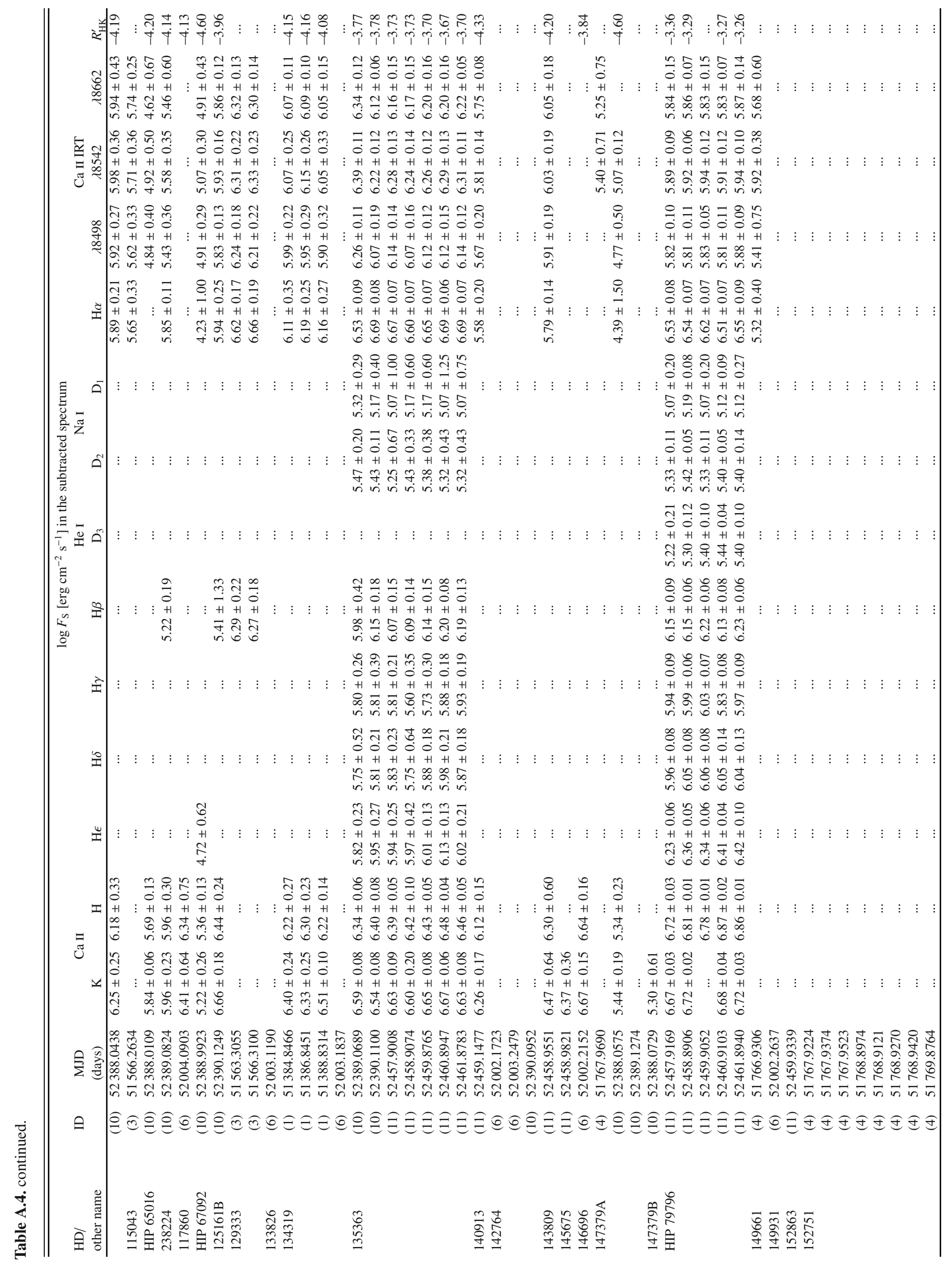

Page 28 of 58 
J. López-Santiago et al.: High-resolution spectroscopic survey of late-type stars

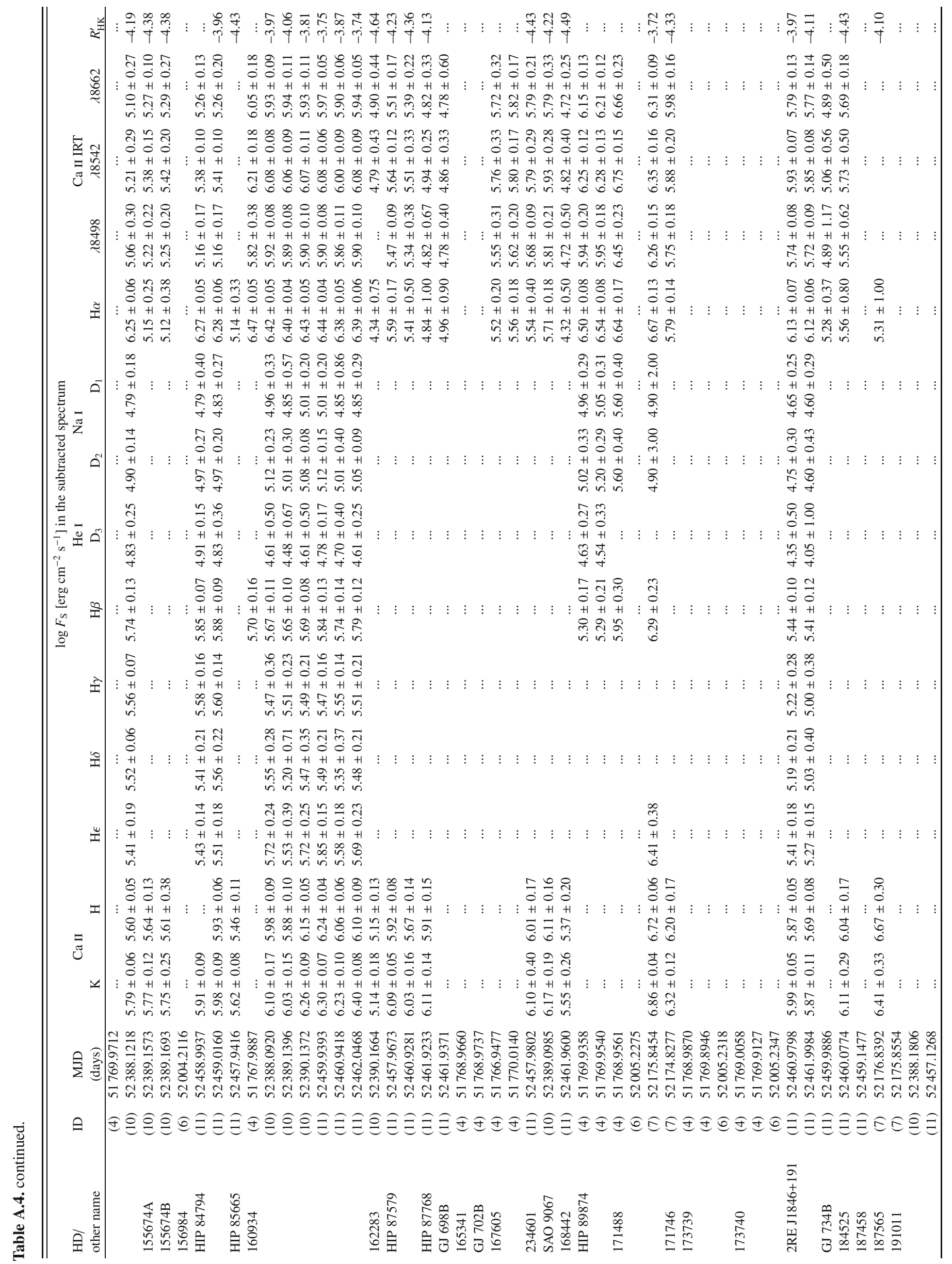


A\&A 514, A97 (2010)

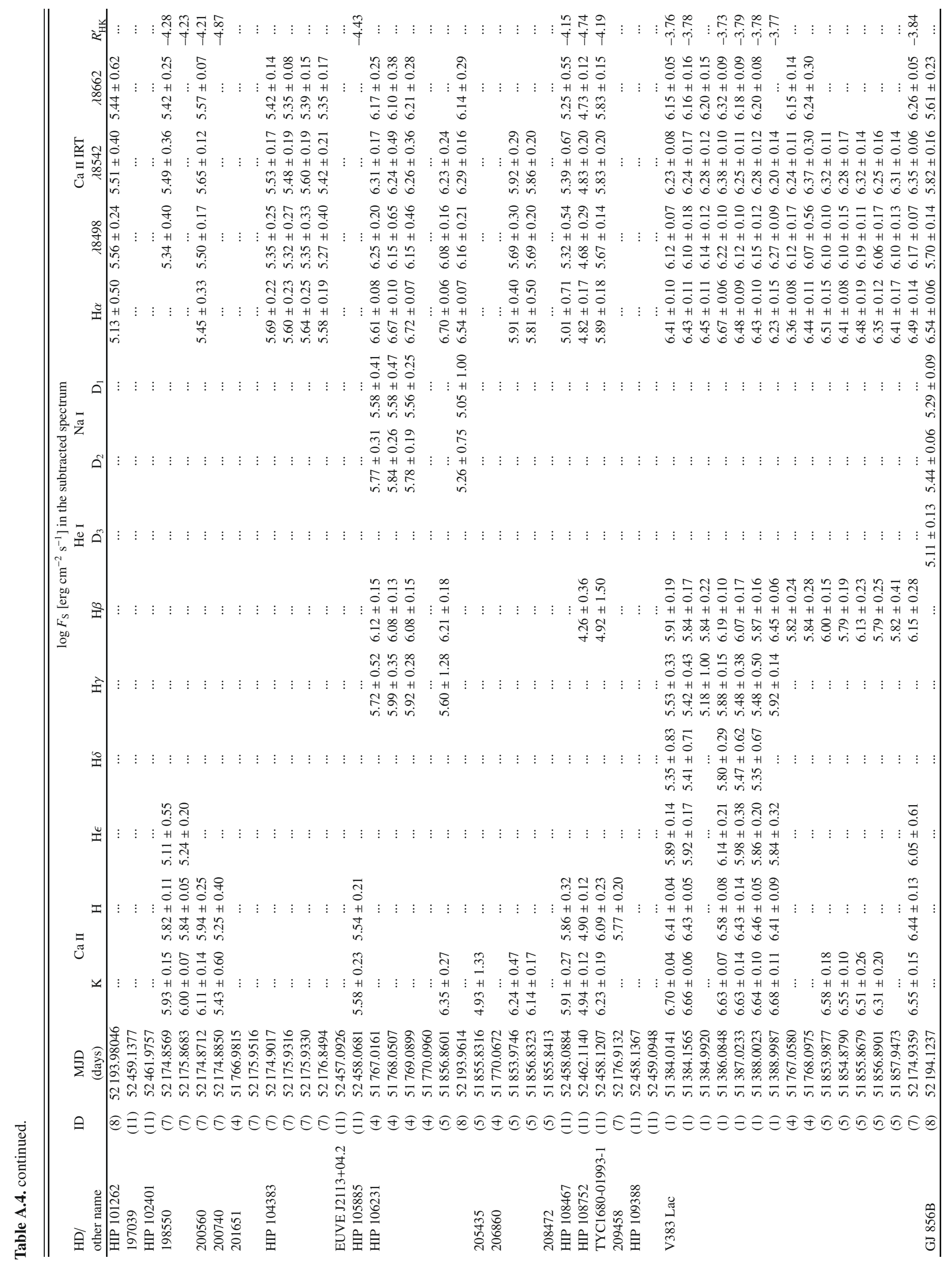

Page 30 of 58 
J. López-Santiago et al.: High-resolution spectroscopic survey of late-type stars

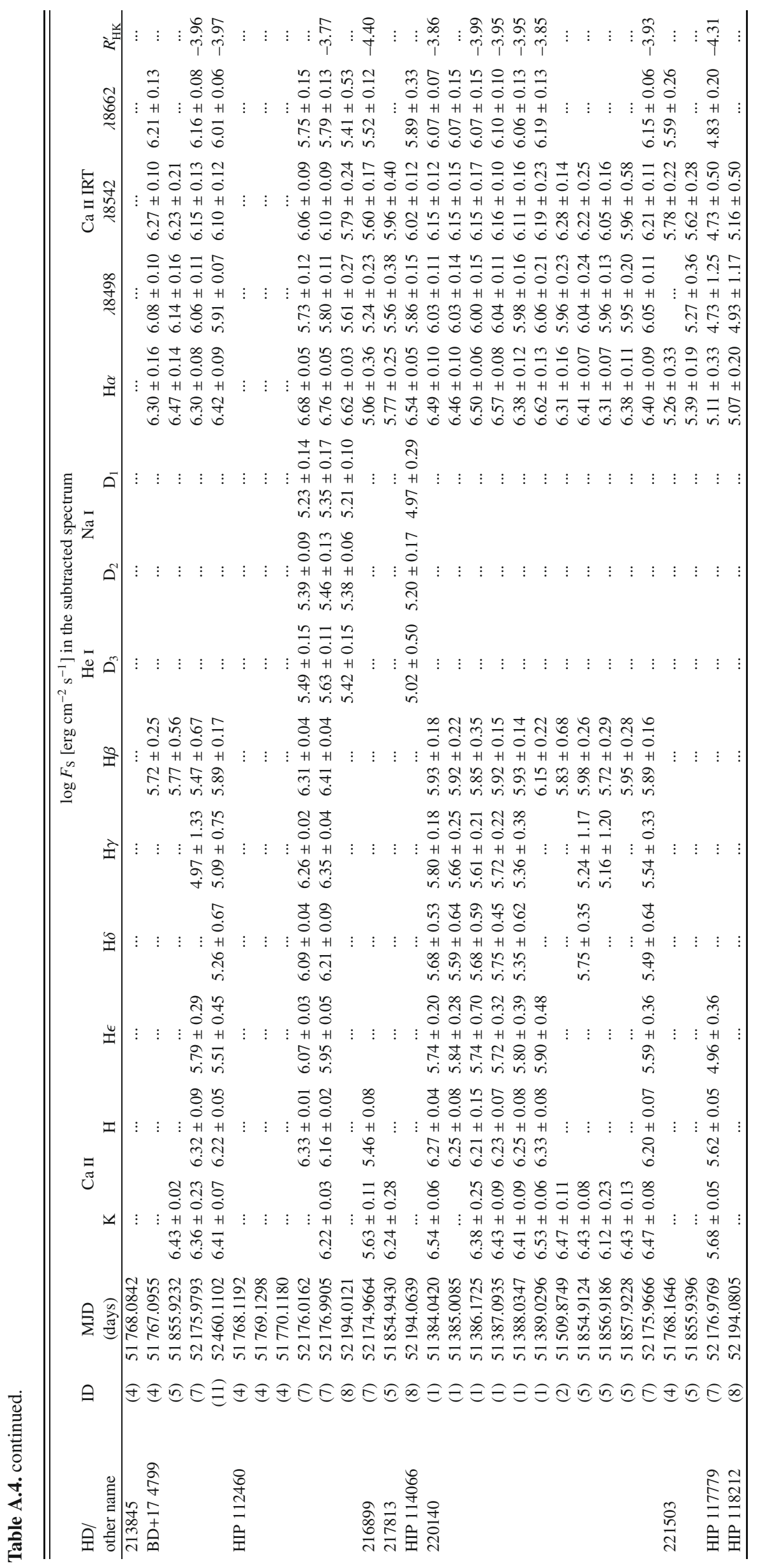



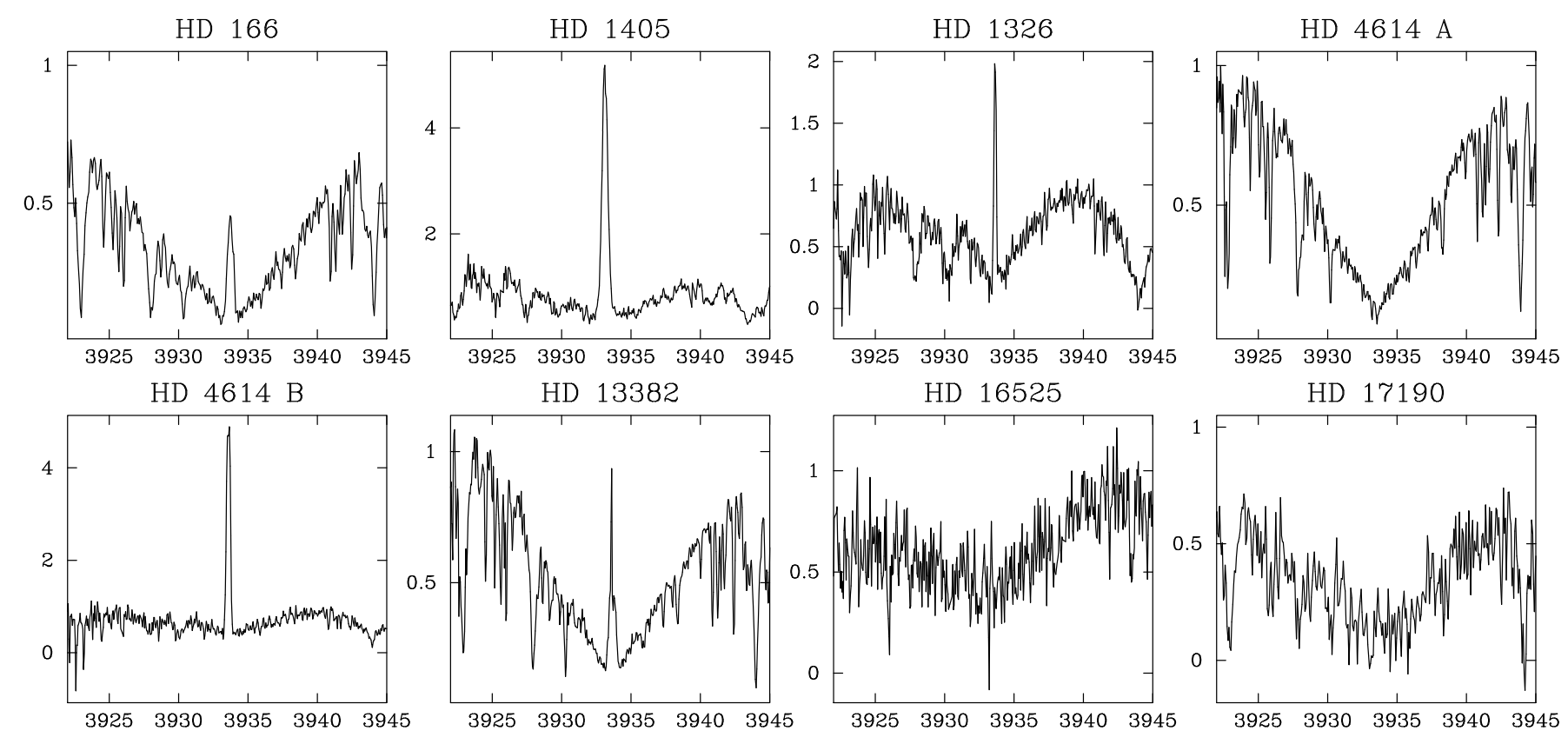

HD 16525
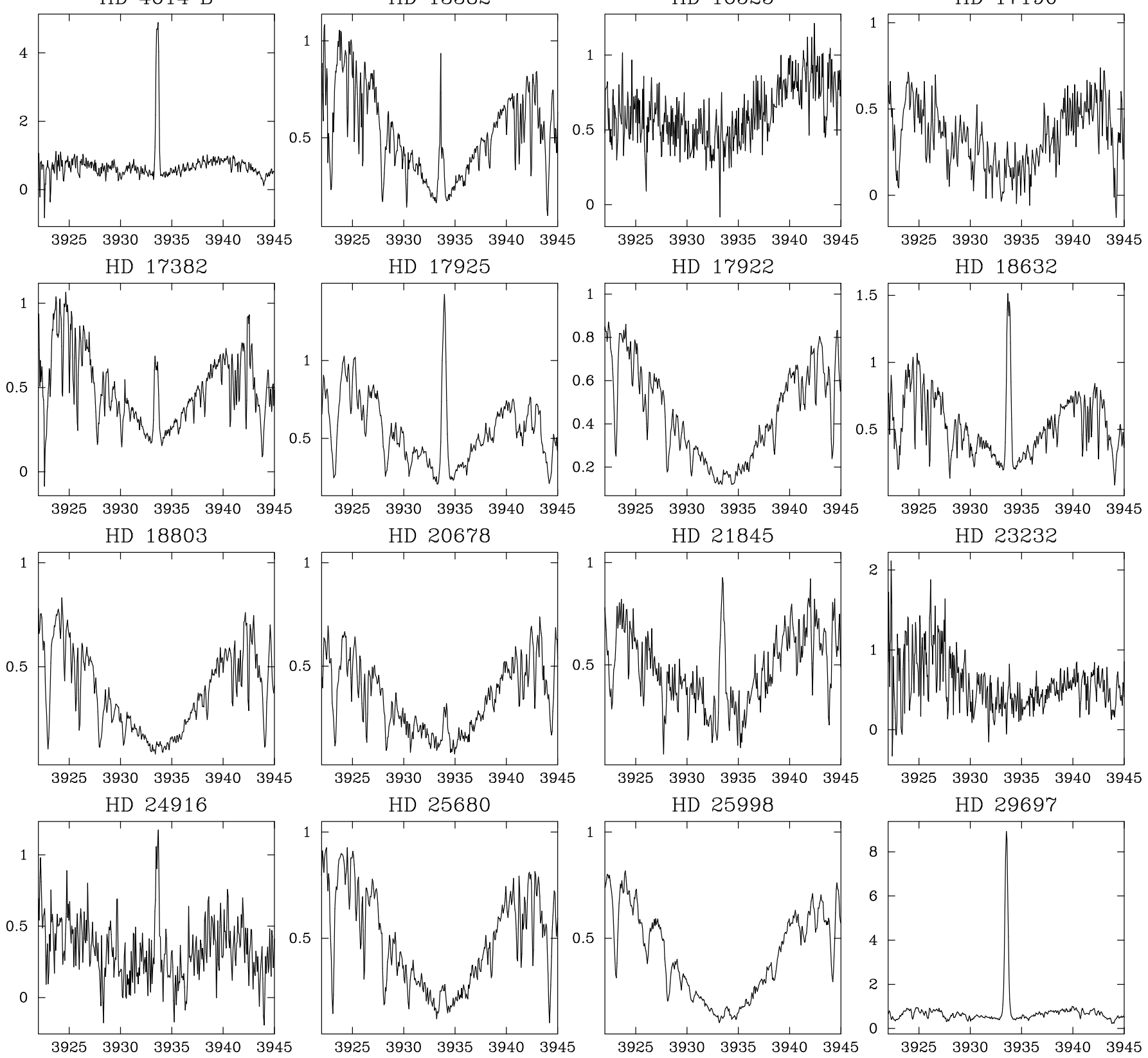

Fig. A.1. Ca II K spectra of the stars of our sample with observations in the $\mathrm{H} \& \mathrm{~K}$ wavelength range. 
J. López-Santiago et al.: High-resolution spectroscopic survey of late-type stars
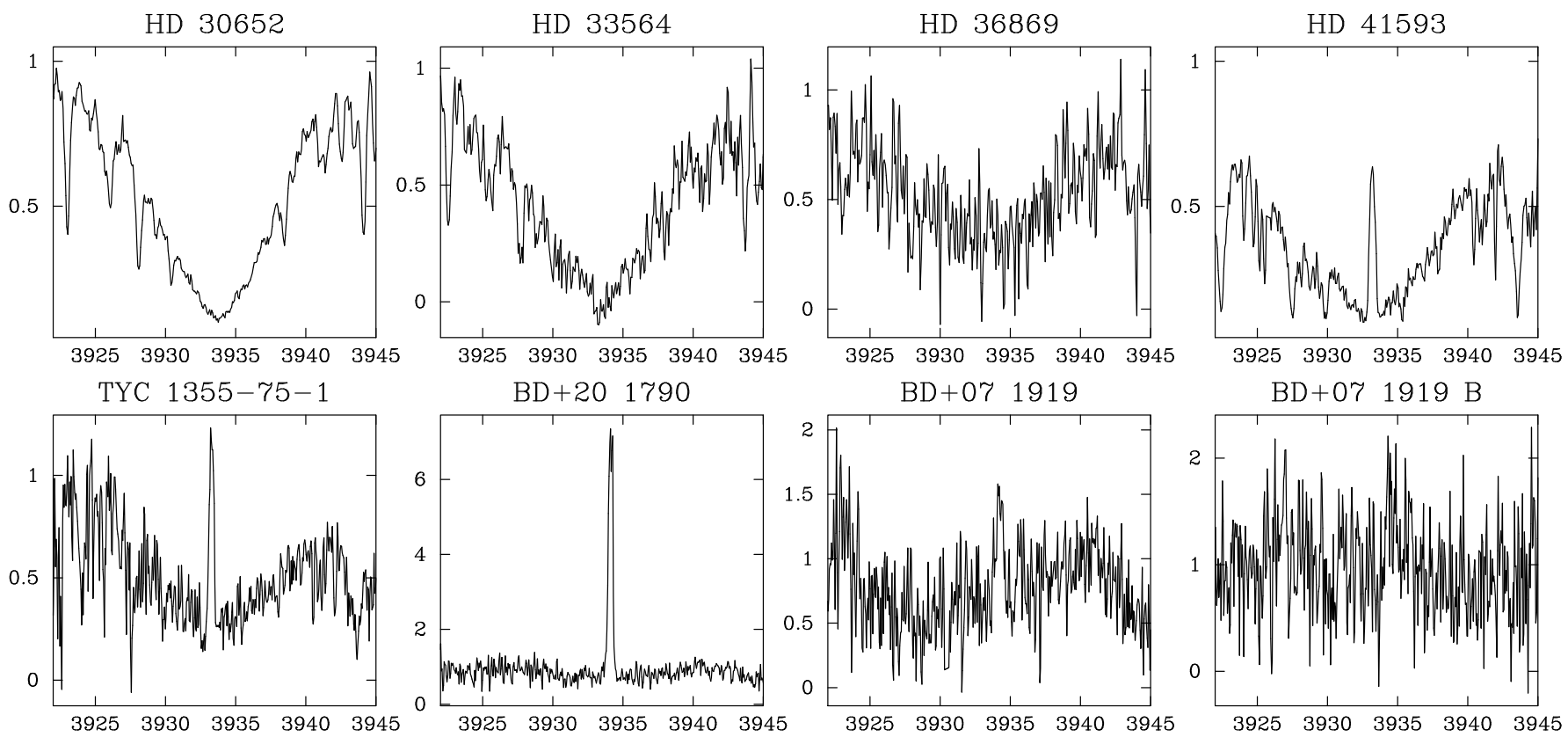

$\mathrm{BD}+071919 \mathrm{~B}$

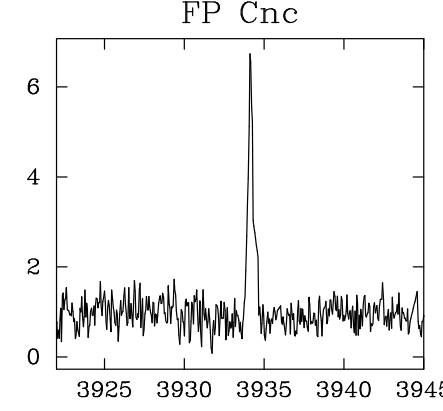

HD 72905

HD 73171
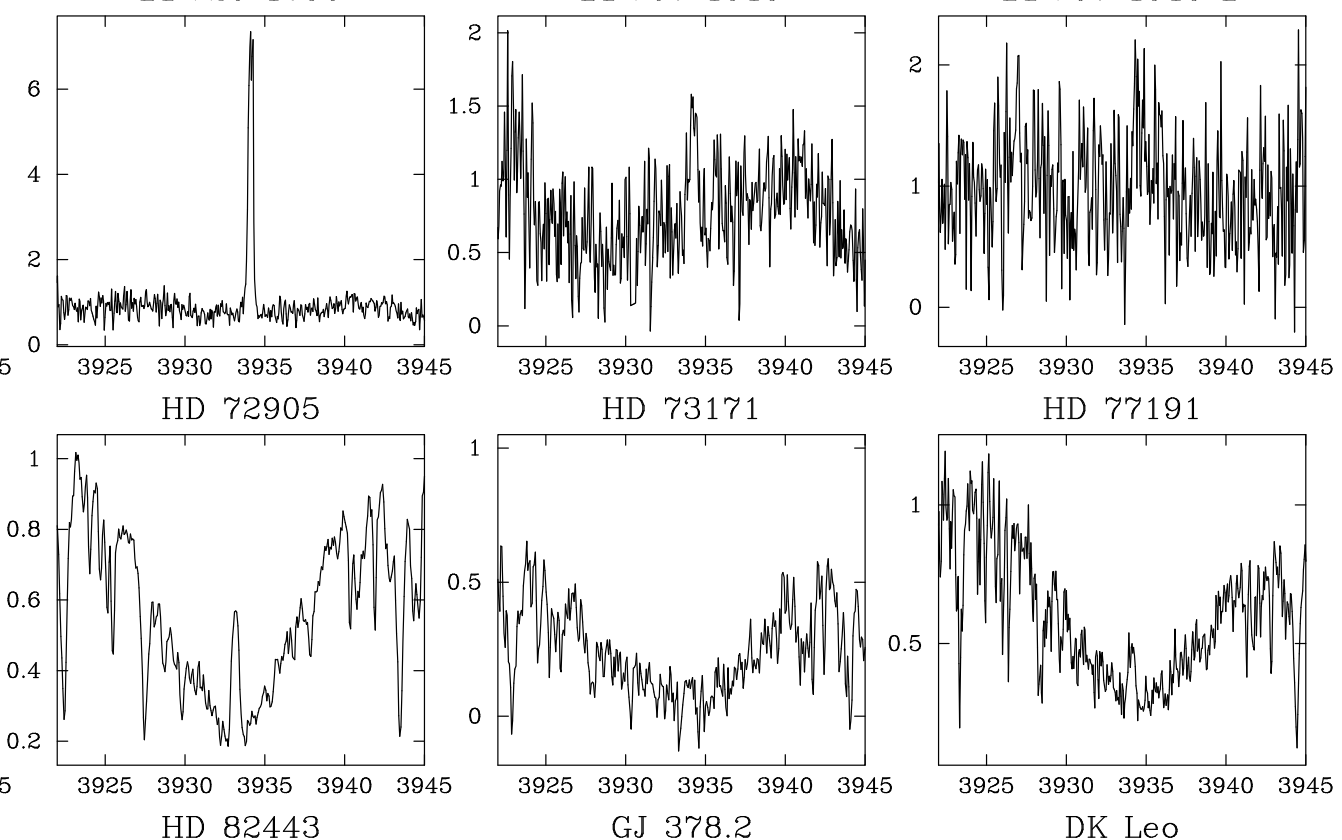

HD 77191
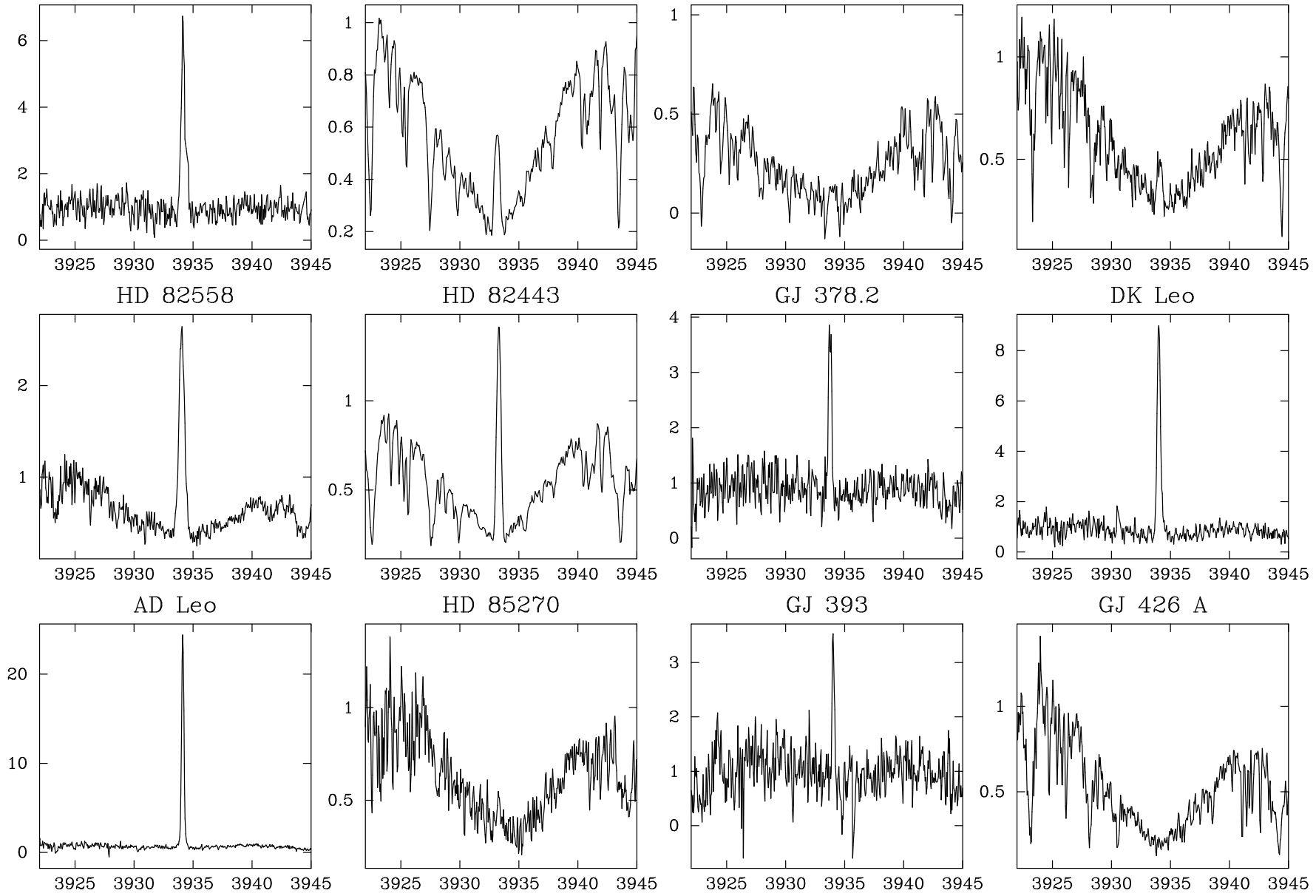

Fig. A.1. continued. 
A\&A 514, A97 (2010)
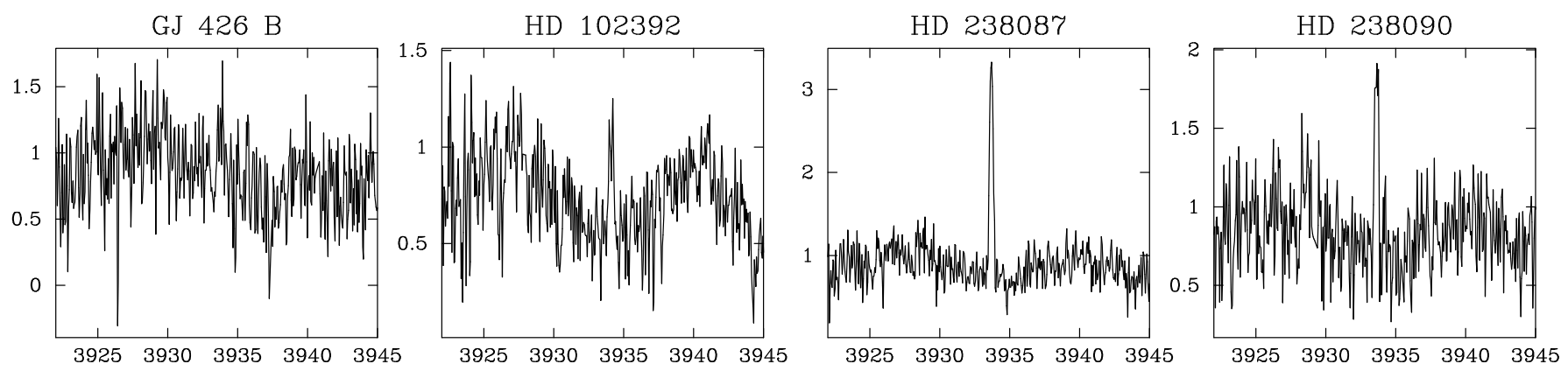

HD 106496

GJ 466

HD 110010
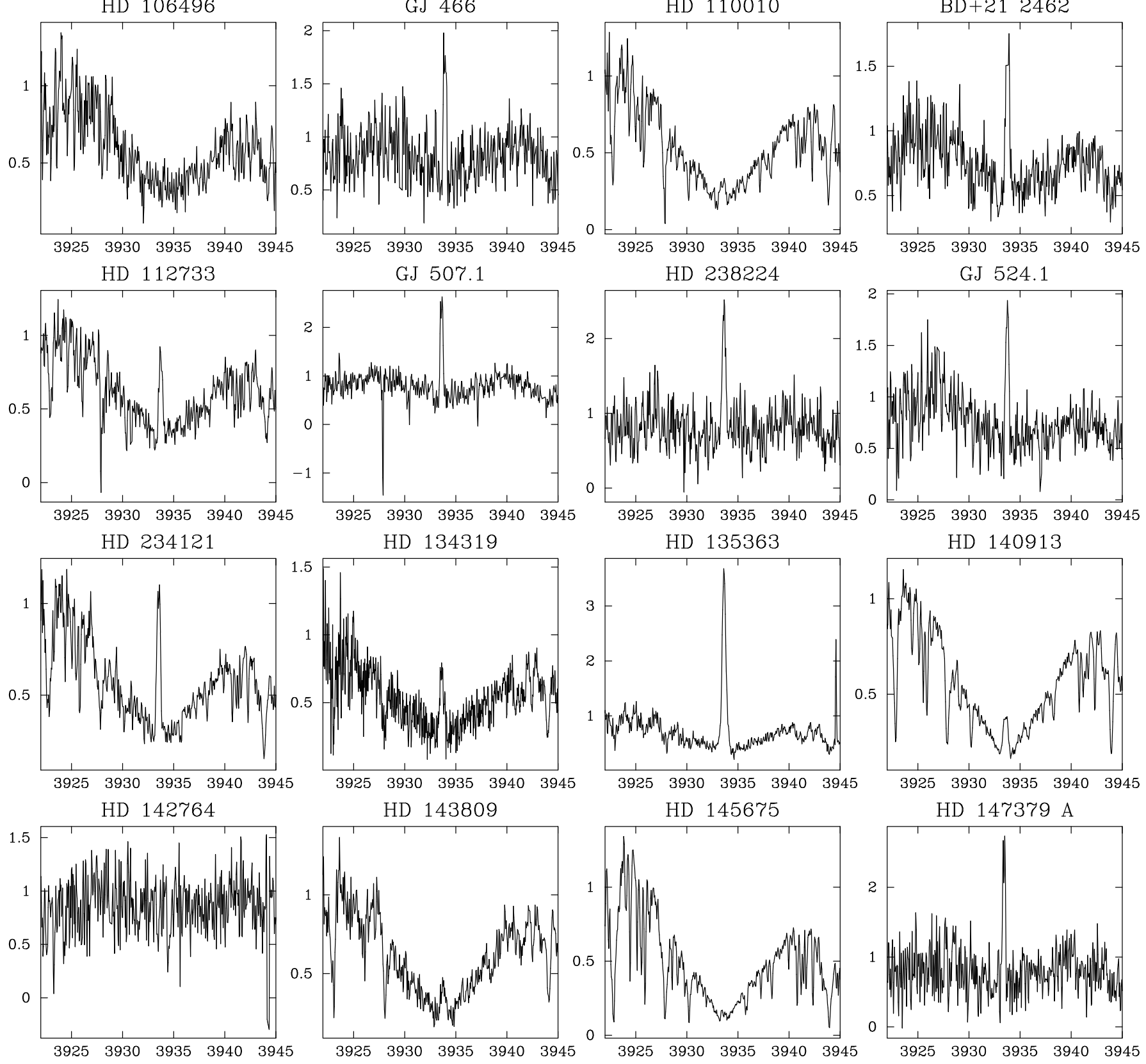

Fig. A.1. continued. 
J. López-Santiago et al.: High-resolution spectroscopic survey of late-type stars
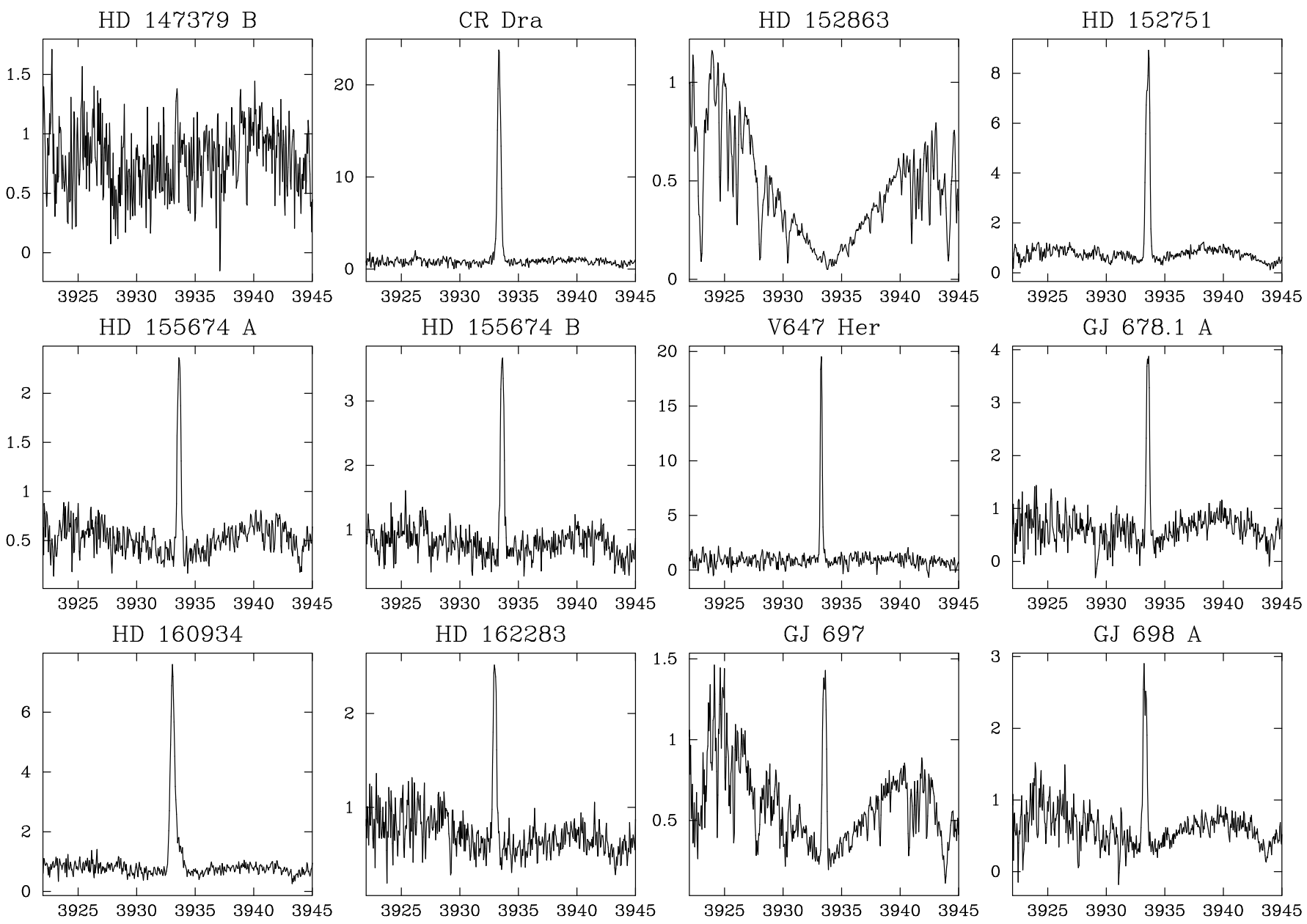

GJ 697

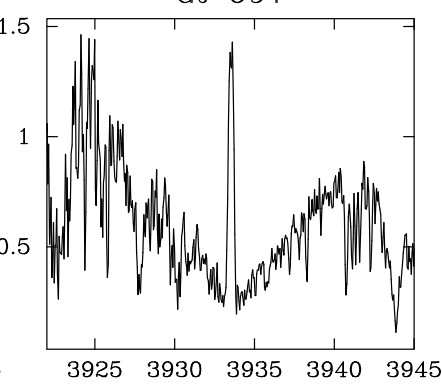

GJ 698 A
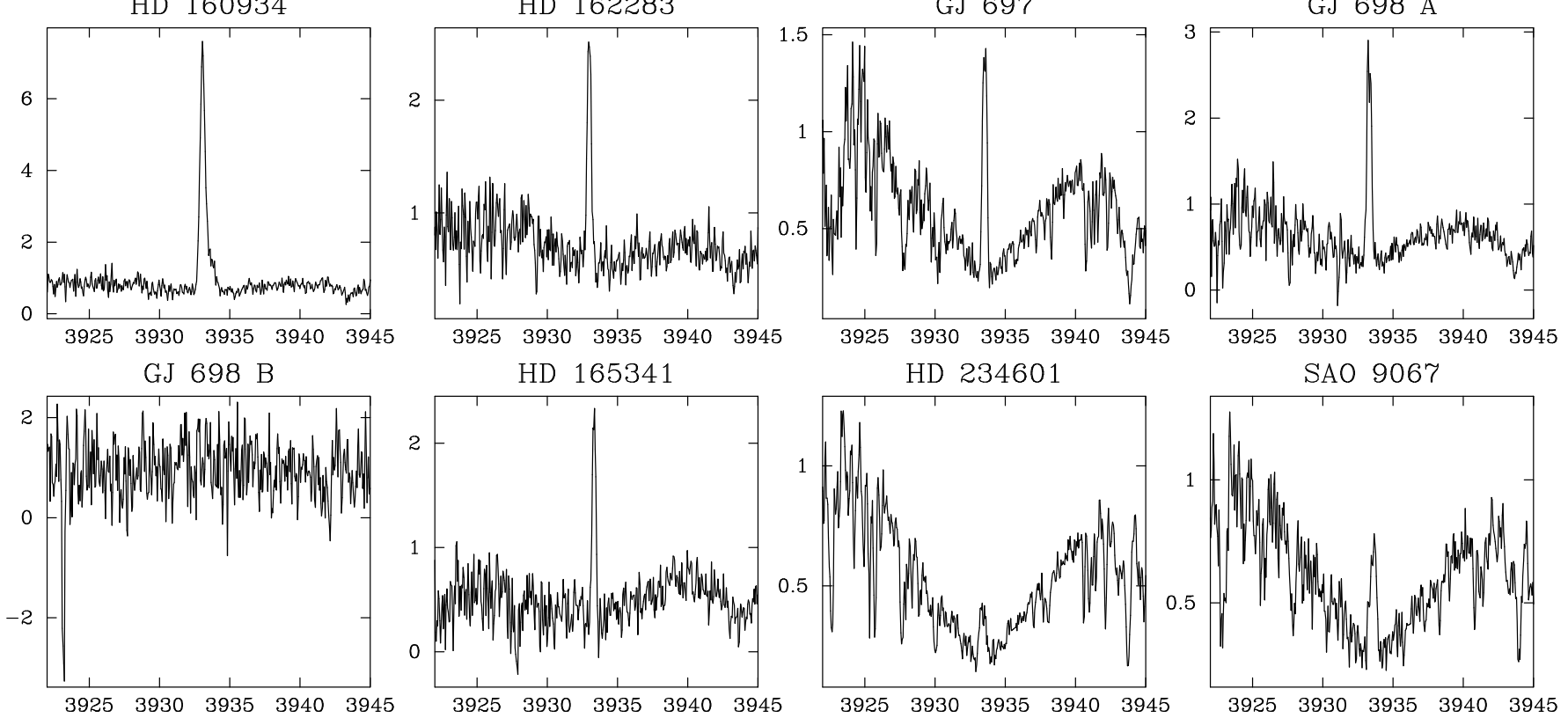

HD 234601
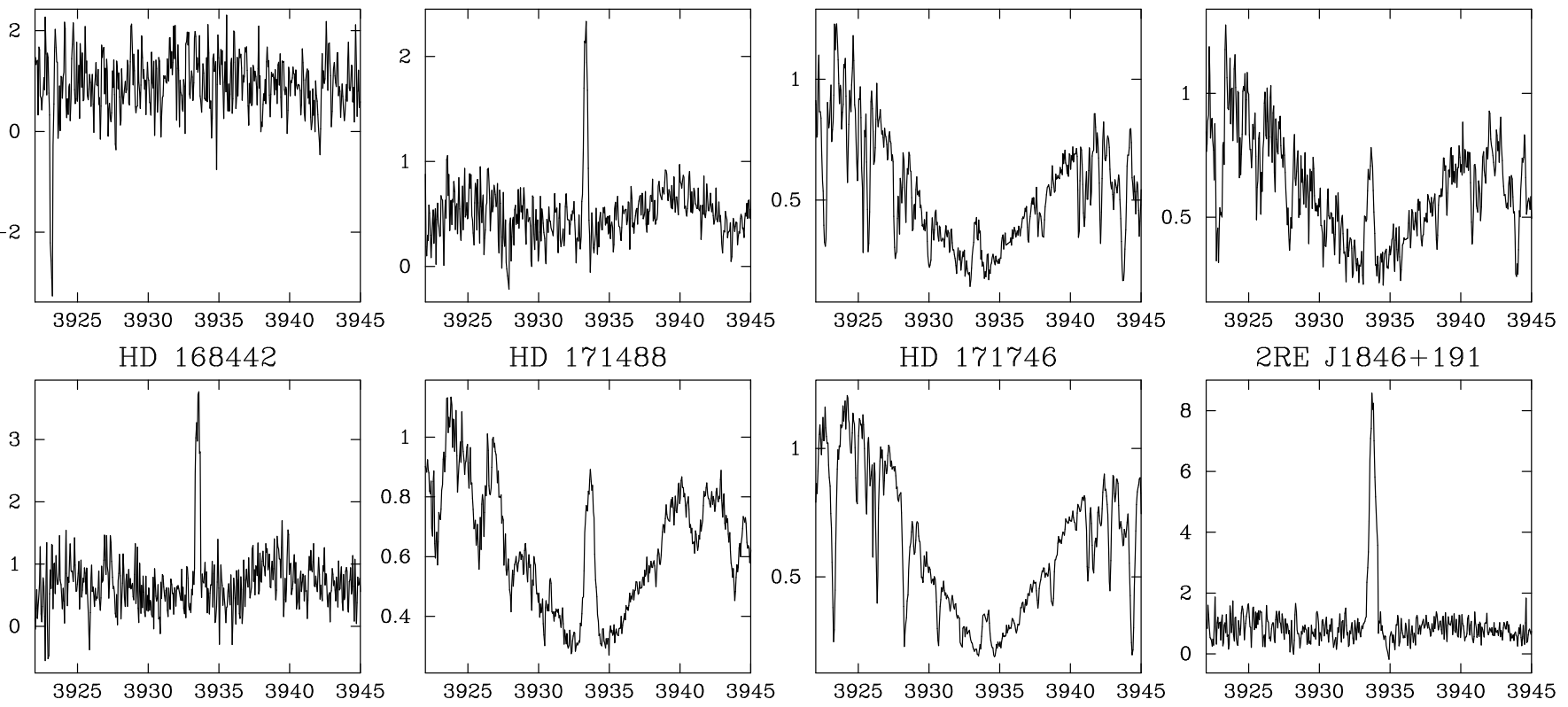

Fig. A.1. continued. 

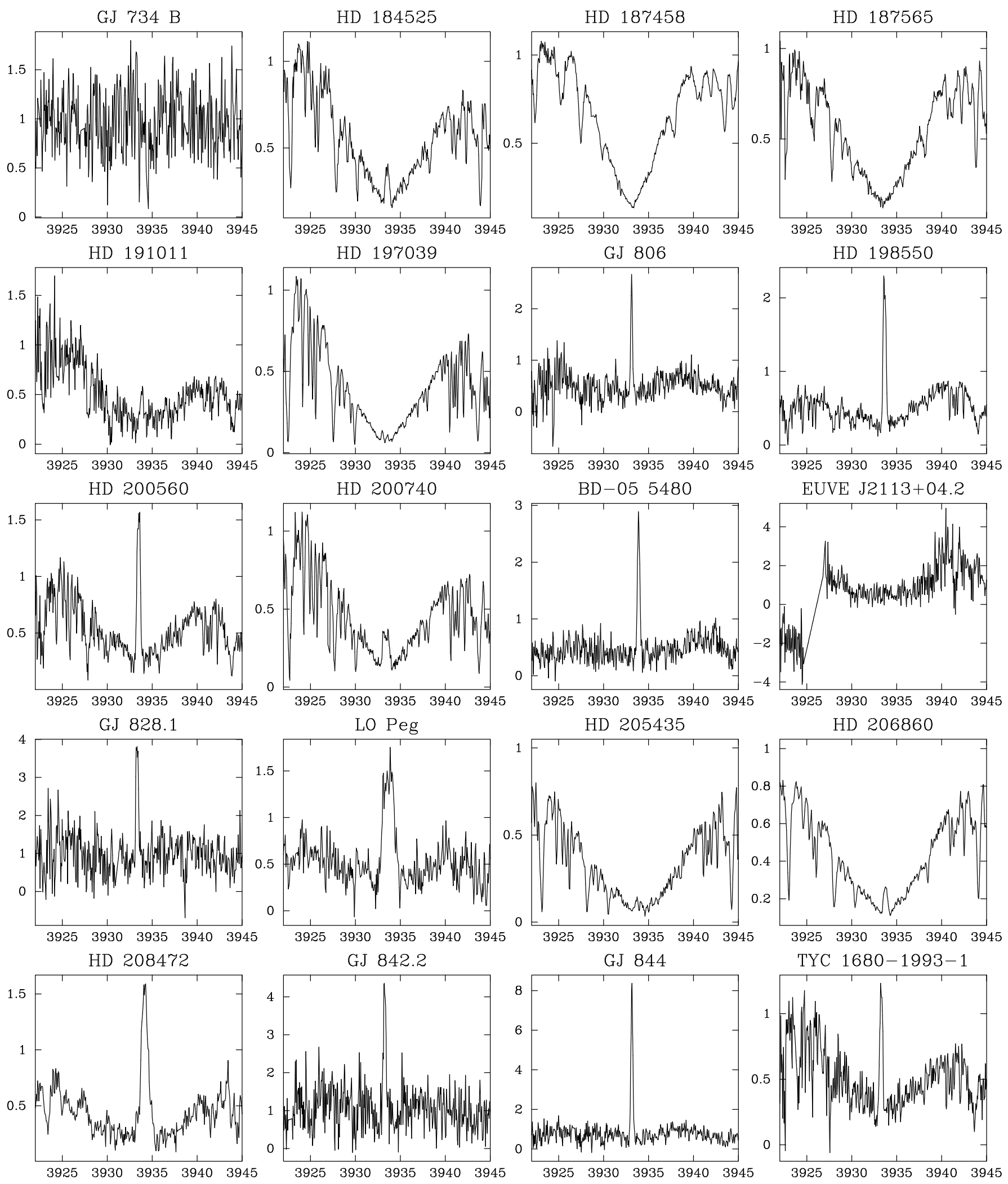

Fig. A.1. continued. 
J. López-Santiago et al.: High-resolution spectroscopic survey of late-type stars
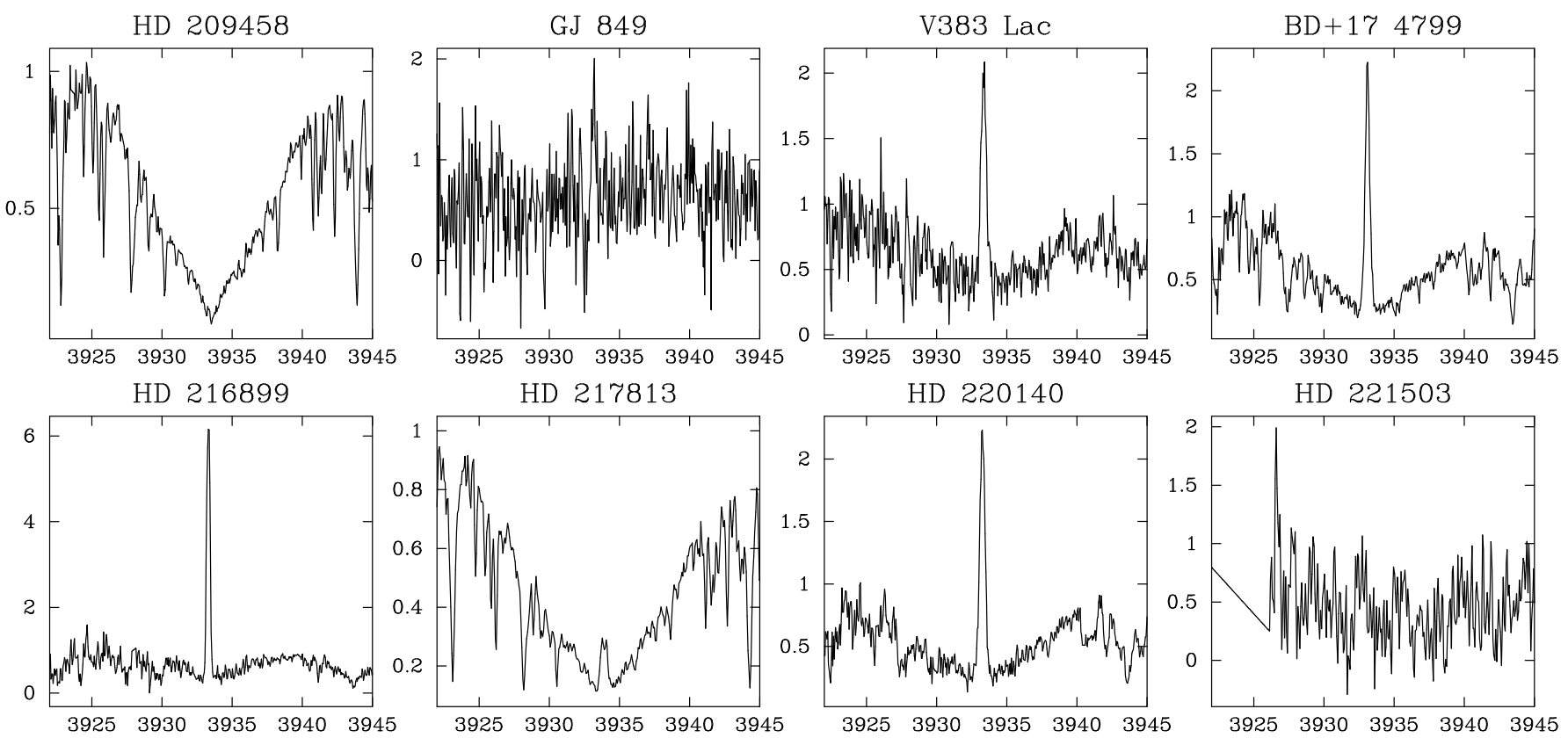
HD 217813

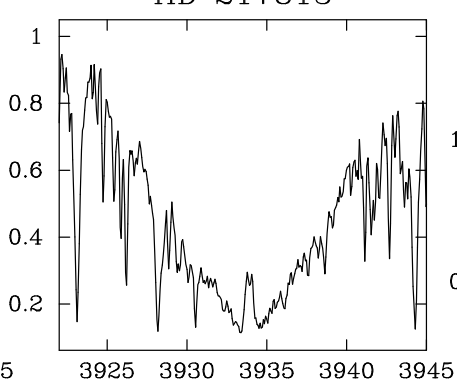

HD 220140 HD 221503
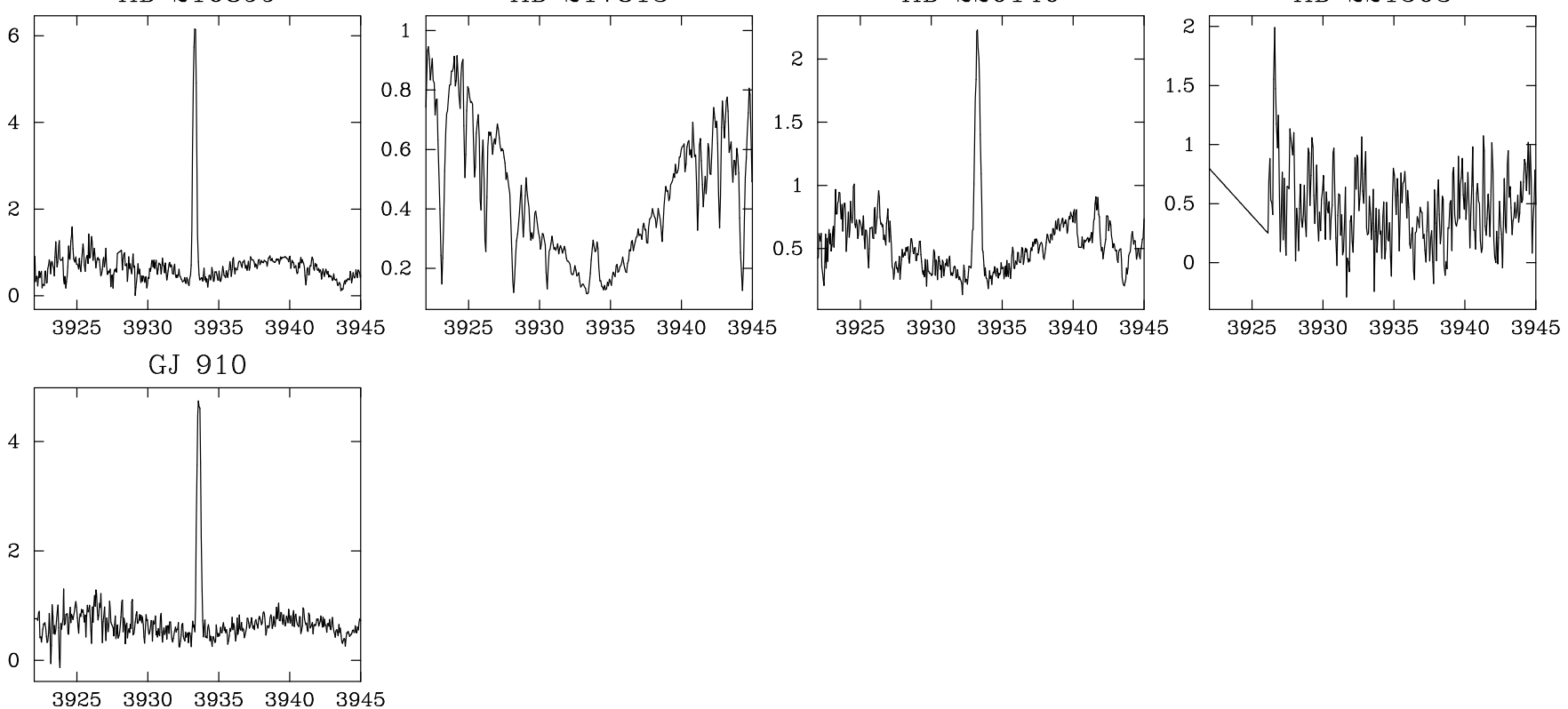

Fig. A.1. continued. 

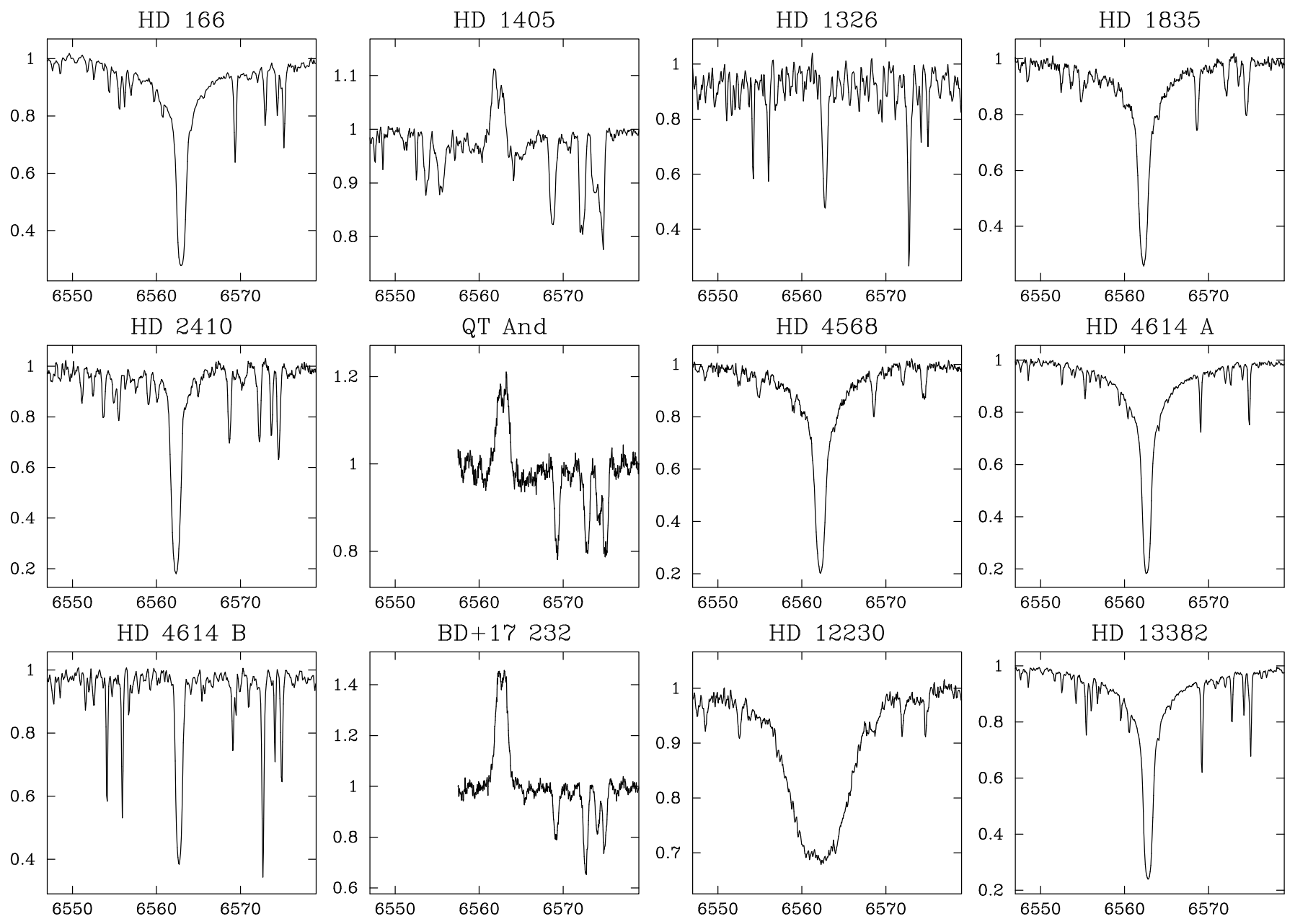

HD 13382
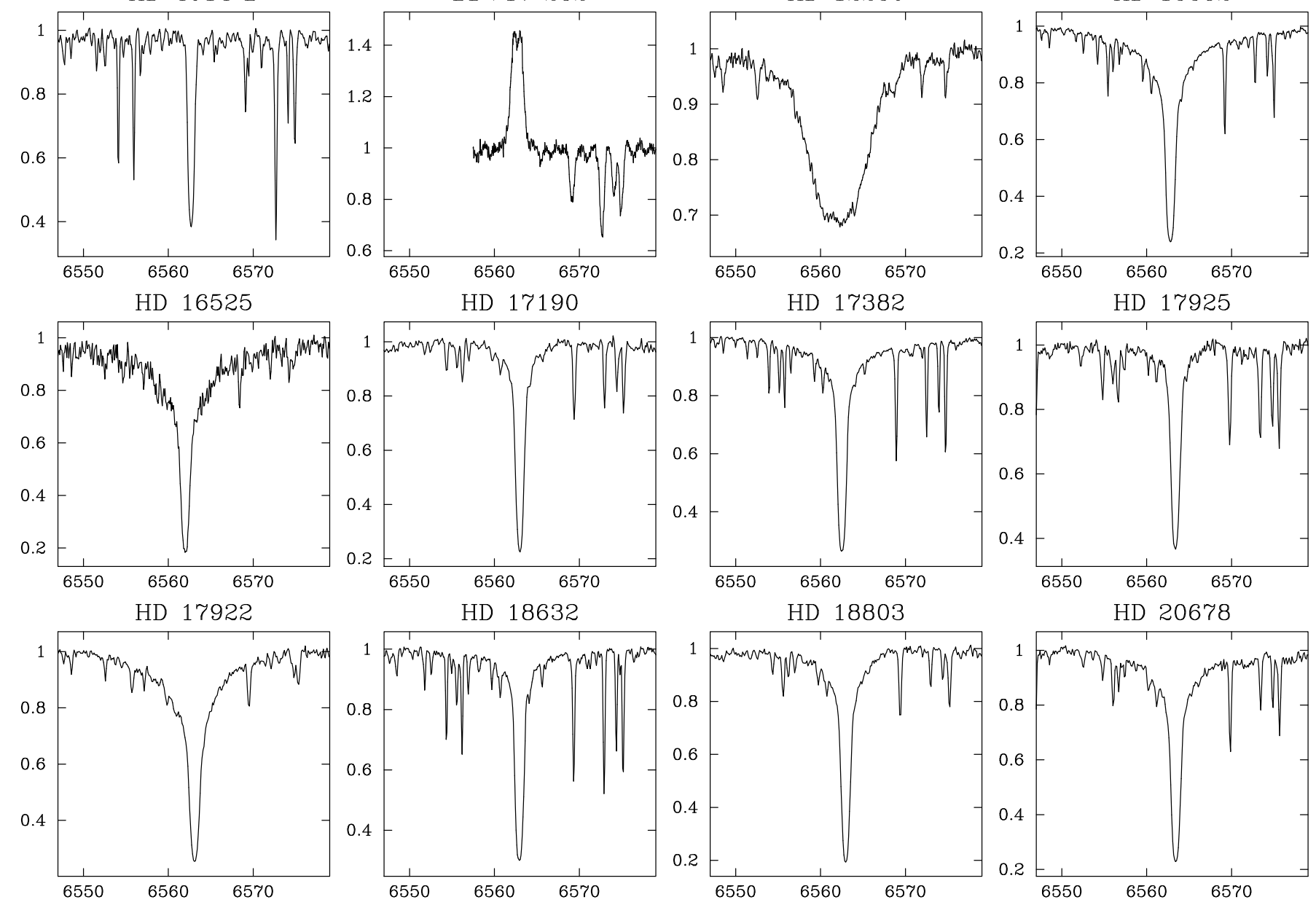

Fig. A.2. $\mathrm{H} \alpha$ spectra of the stars of our sample with observations in $\mathrm{H} \alpha$. 
J. López-Santiago et al.: High-resolution spectroscopic survey of late-type stars

HD 21845

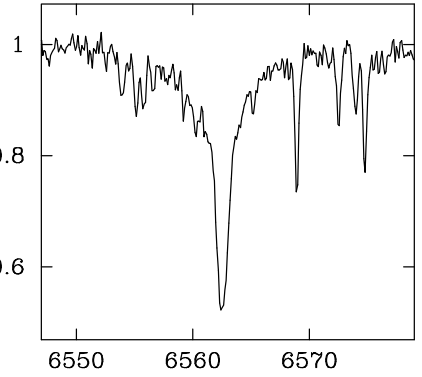

HD 25680

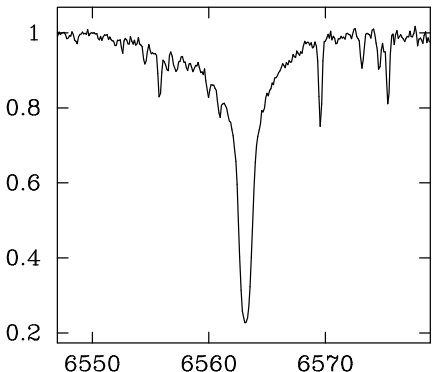

HD 30652

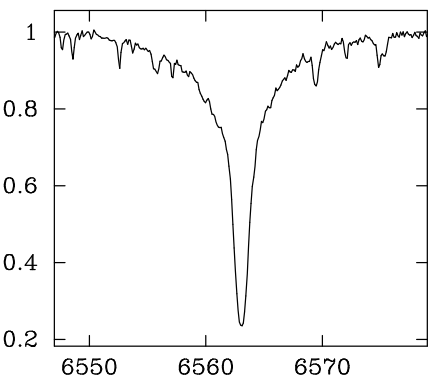

HD 233153

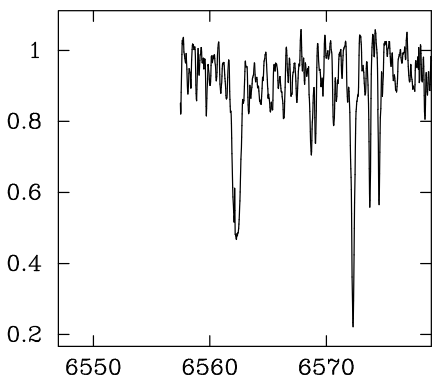

$\mathrm{BD}+071919$

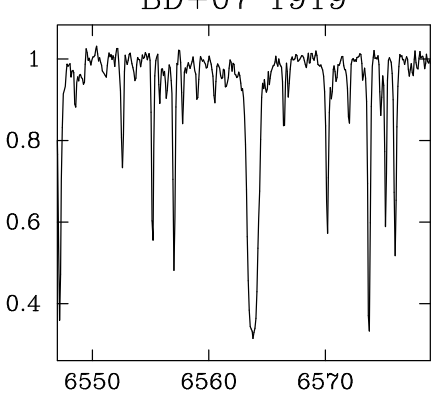

Fig. A.2. continued.
HD 23232
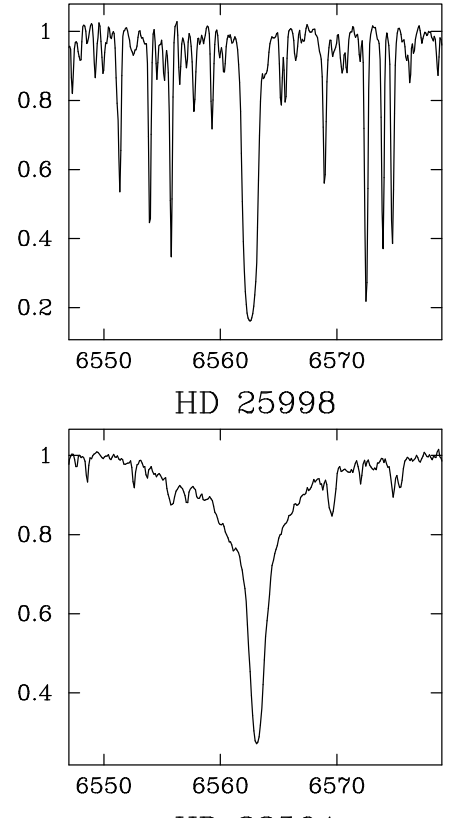

HD 33564

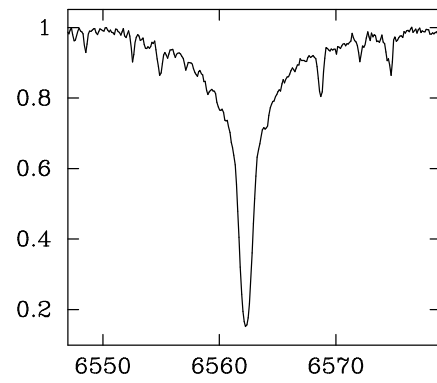

HD 41593

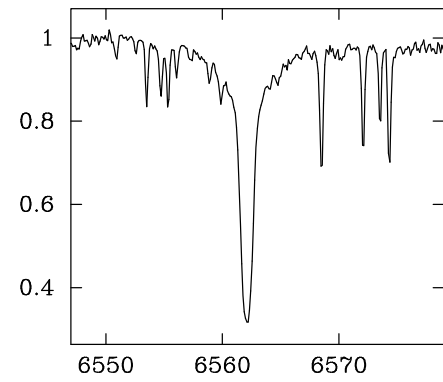

$\mathrm{BD}+071919 \mathrm{~B}$

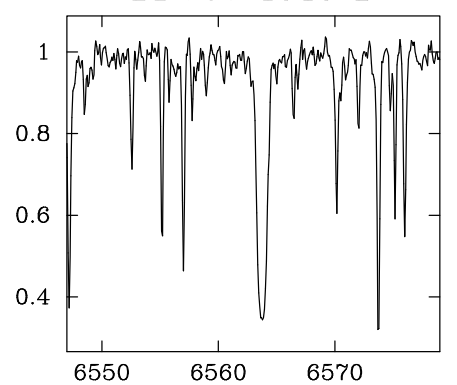

HD 24916
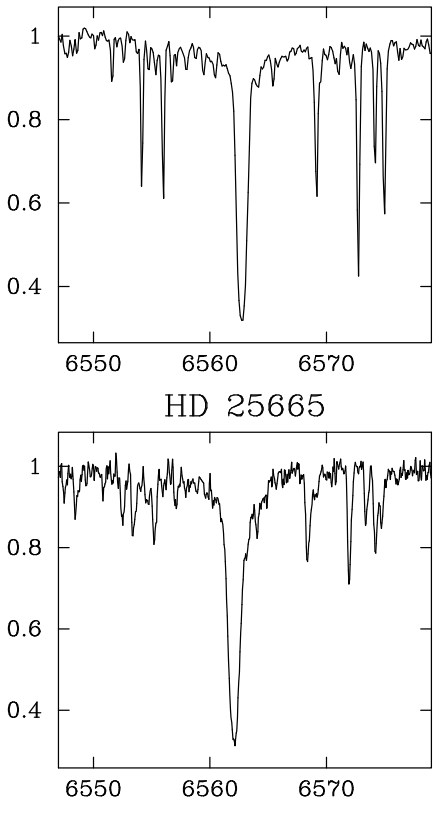

HD 36869

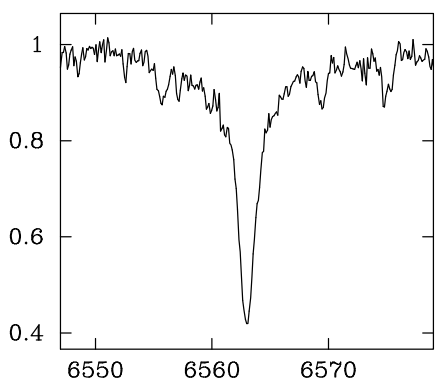

TYC $1355-75-1$

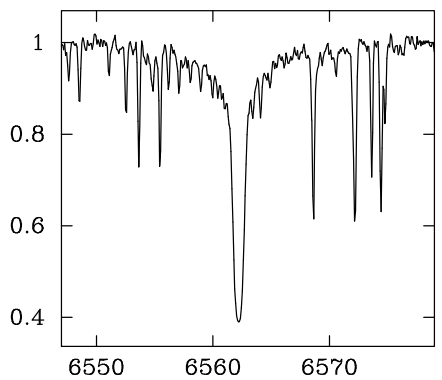

FP Cnc

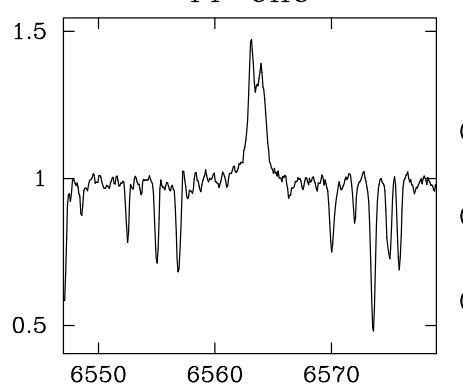

HD 25457

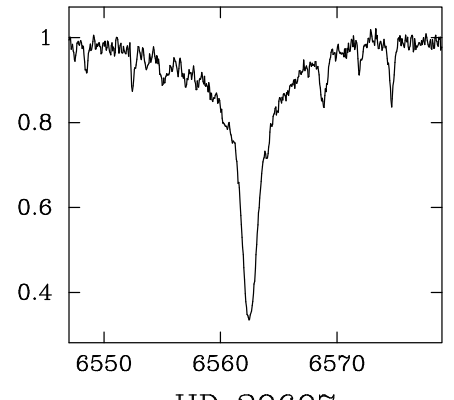

HD 29697

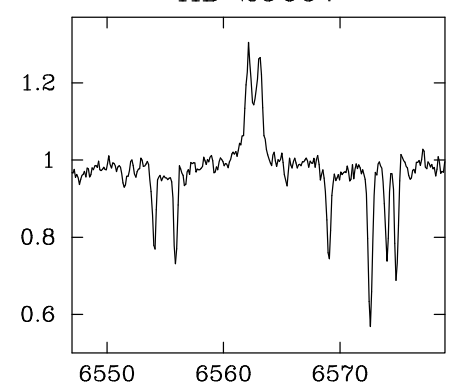

HD 37394

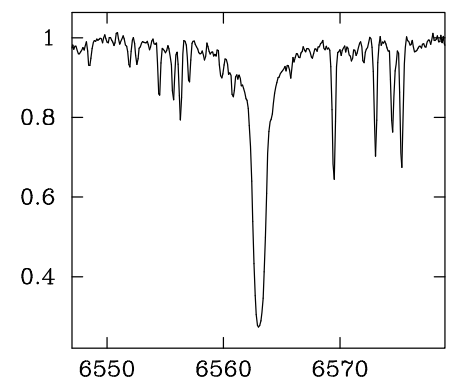

$\mathrm{BD}+20 \quad 1790$

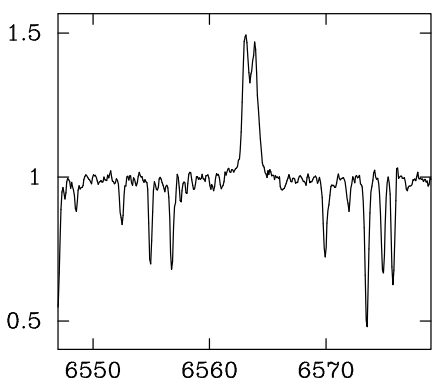

HD 72905

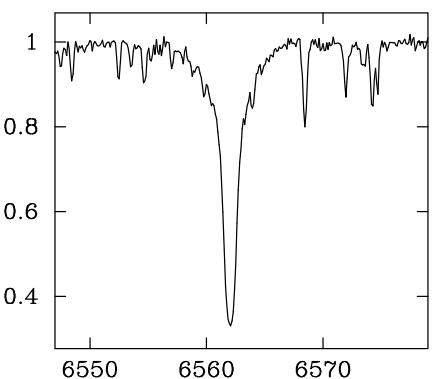



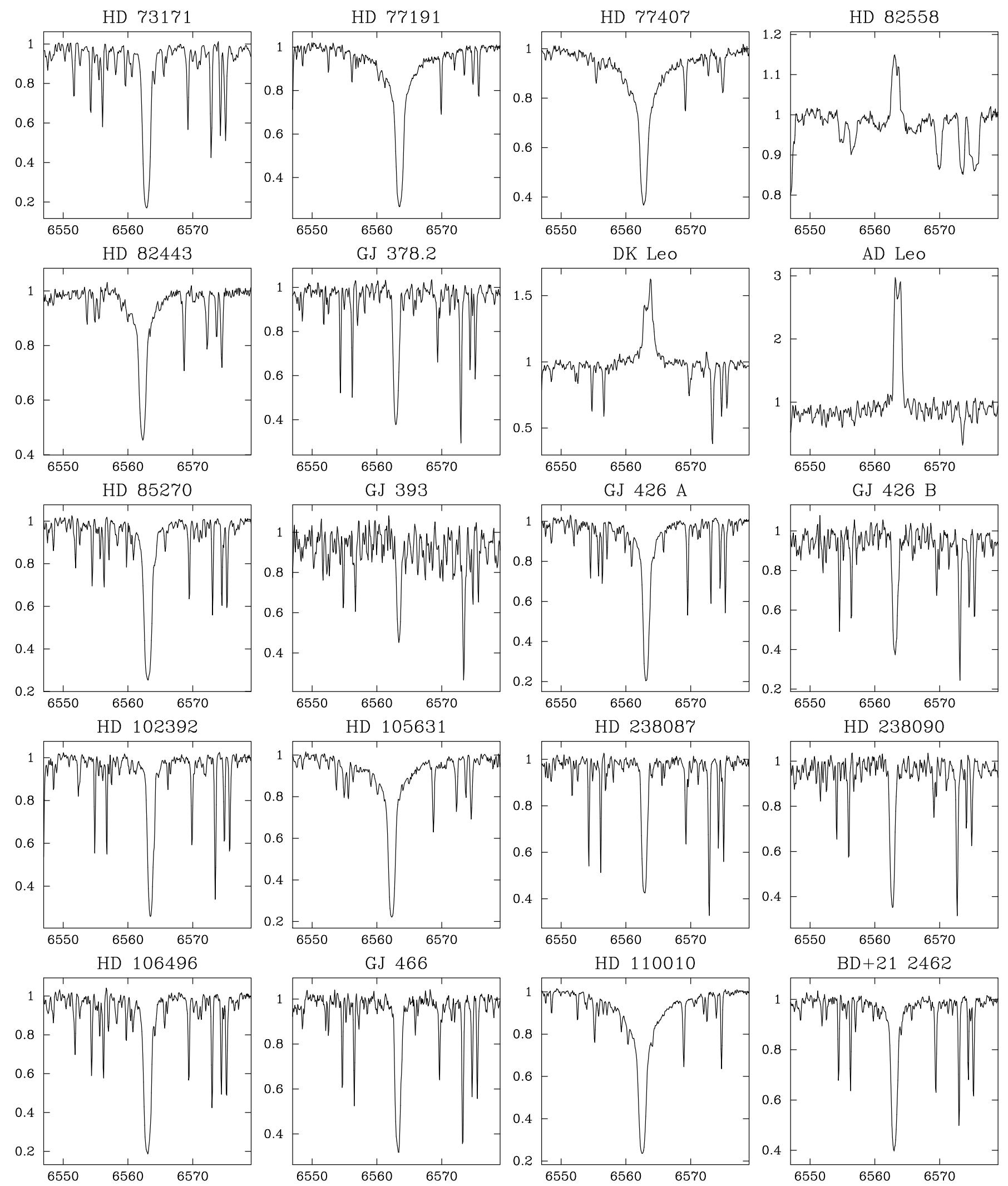

Fig. A.2. continued. 
J. López-Santiago et al.: High-resolution spectroscopic survey of late-type stars

HD 112733

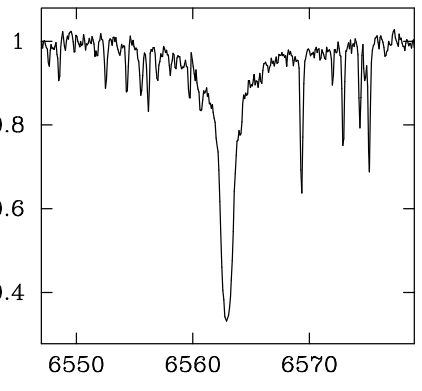

GJ 524.1

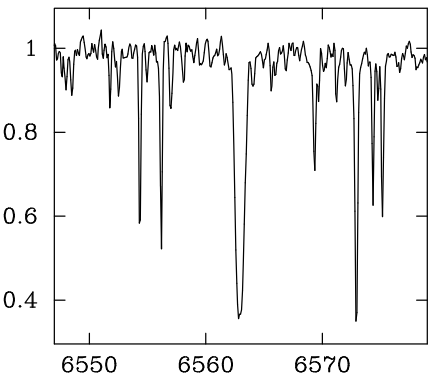

HD 135363

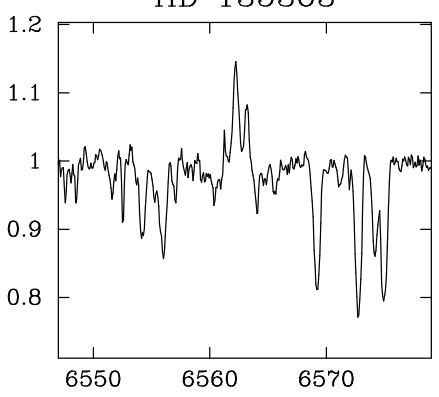

HD 145675

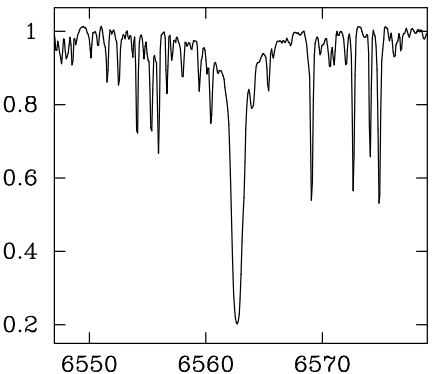

HD 149661

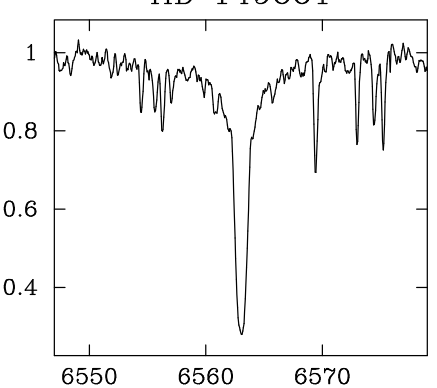

HD 115043

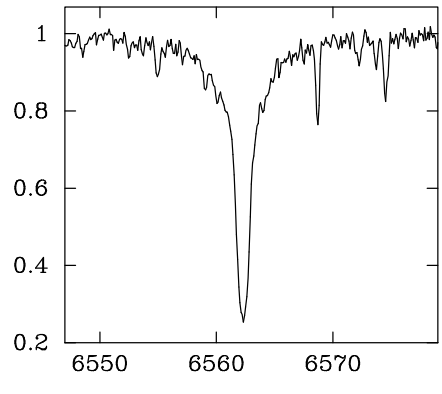

HD 234121

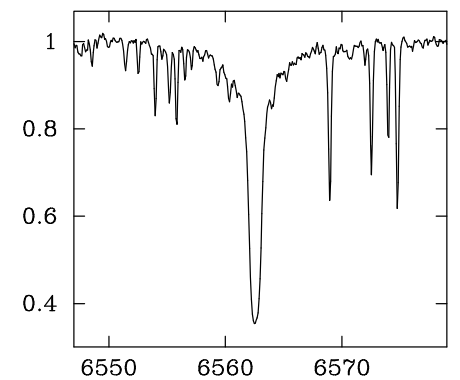

HD 140913

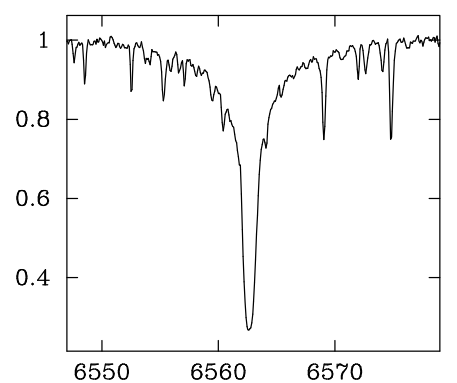

HD 147379 A

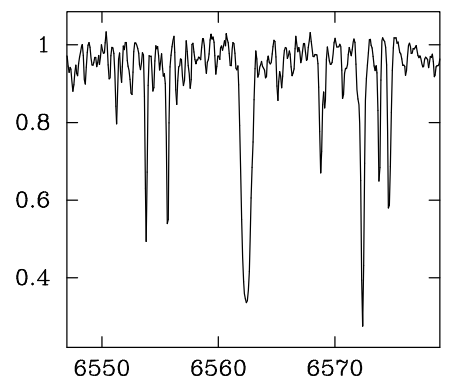

HD 152863

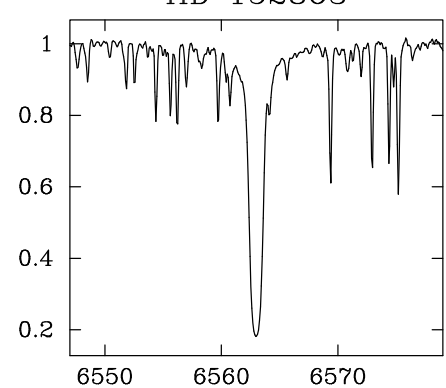

GJ 507.1
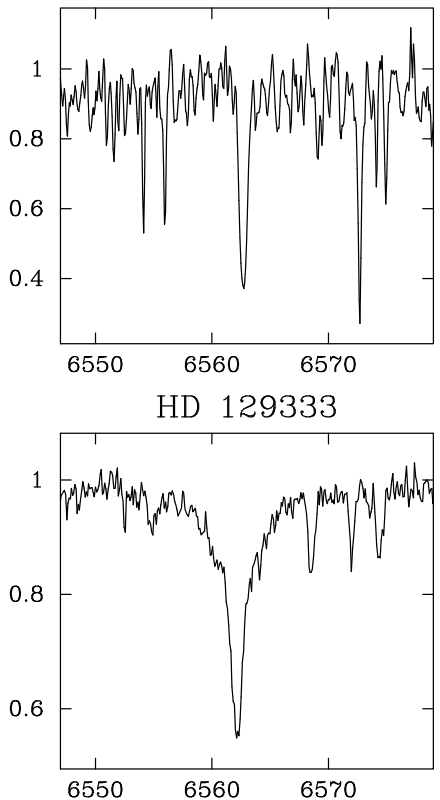

HD 142764

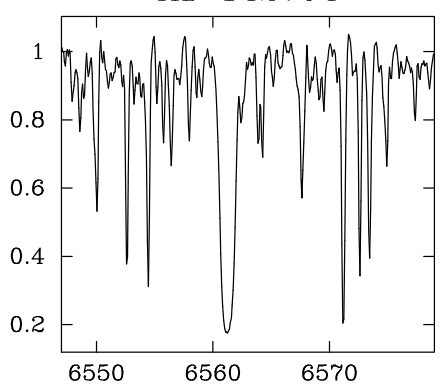

HD 147379 B

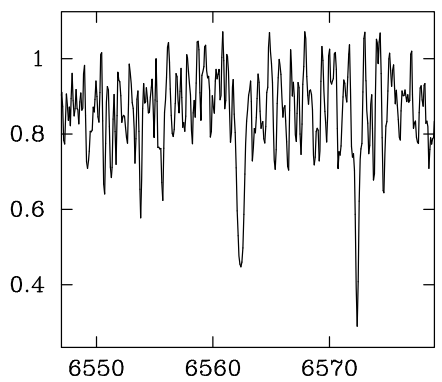

HD 152751

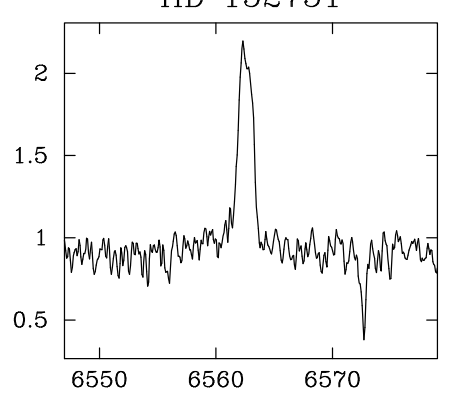

HD 238224

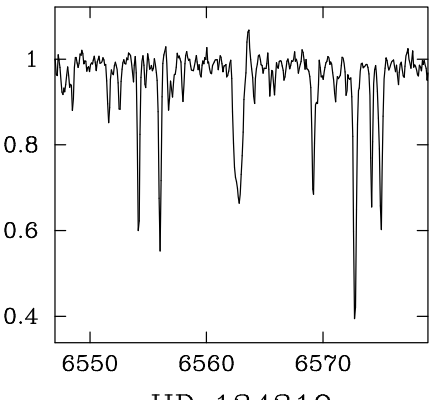

HD 134319

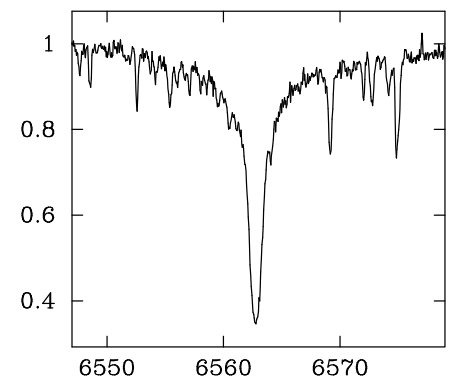

HD 143809

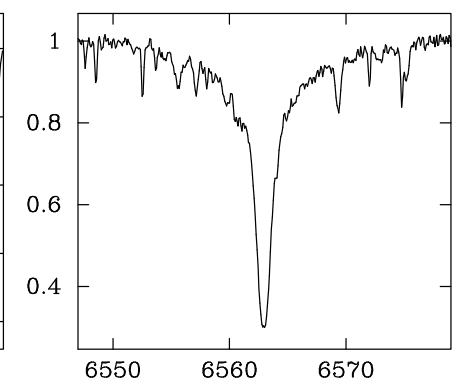

CR Dra

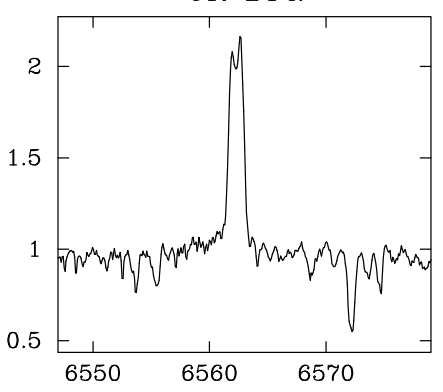

HD 155674 A

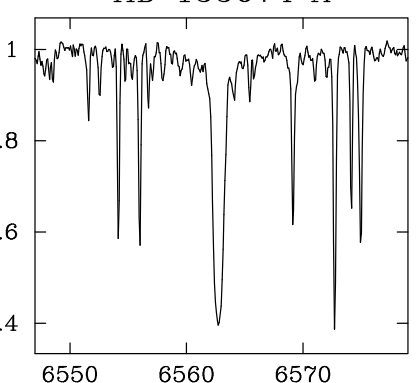

Fig. A.2. continued. 
A\&A 514, A97 (2010)

HD 155674 B
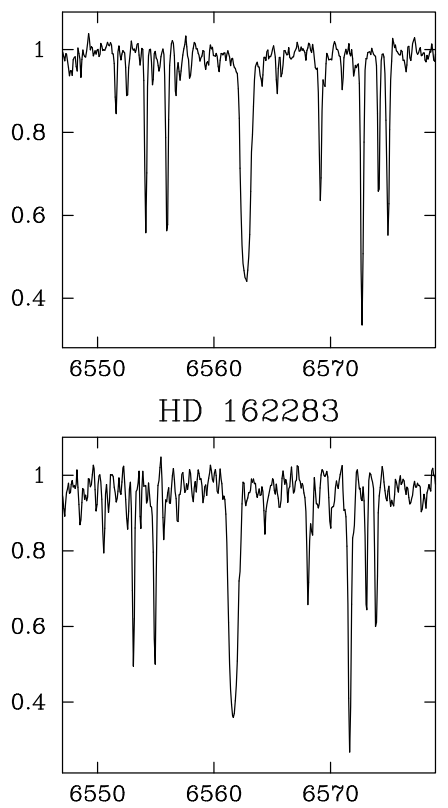

HD 165341

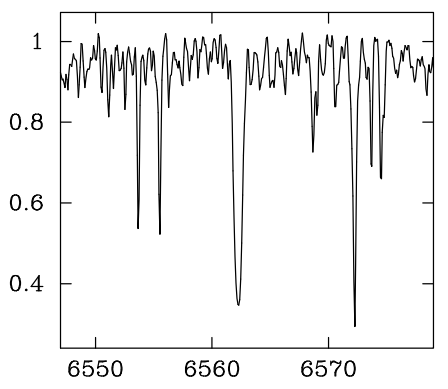

HD 168442

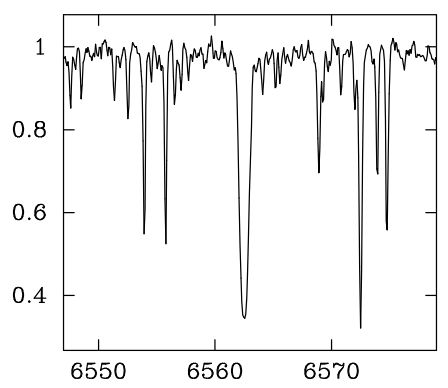

HD 173739

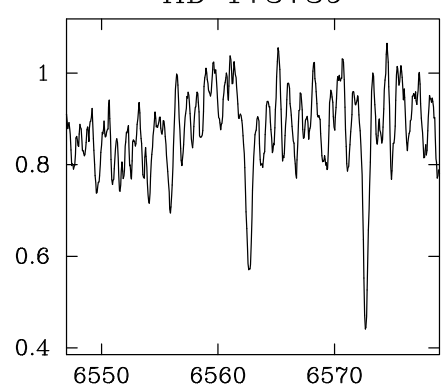

Fig. A.2. continued.
V647 Her
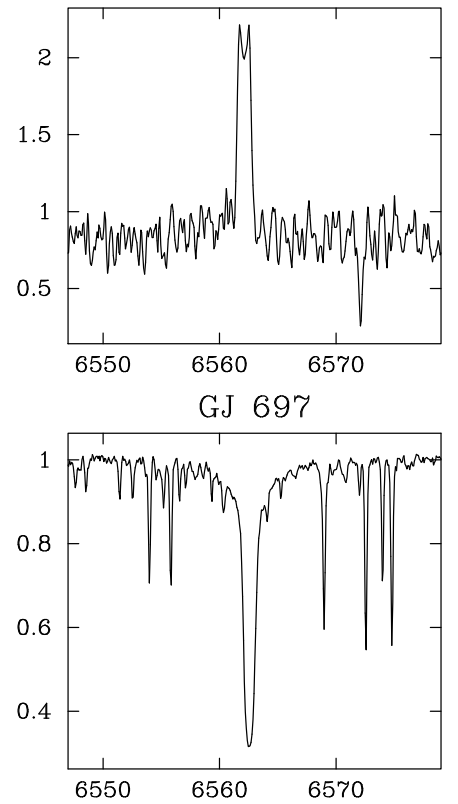

HD 167605

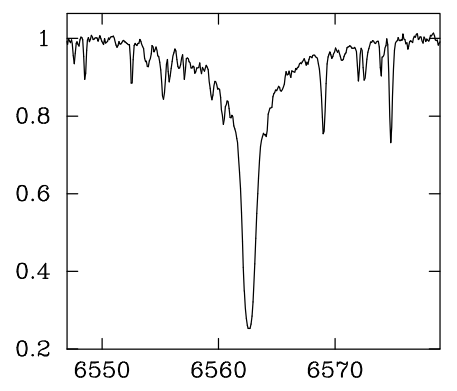

FK Ser

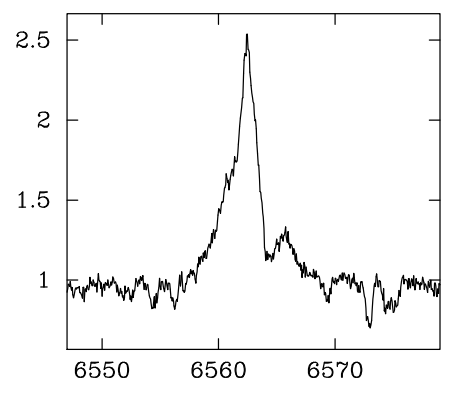

HD 173740

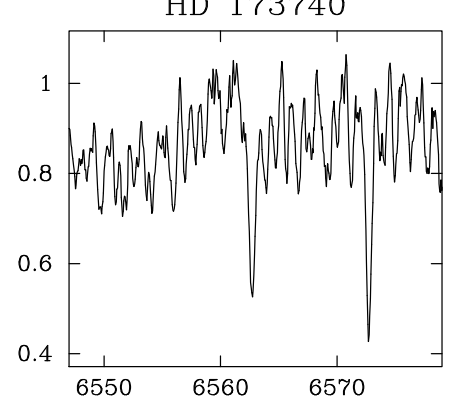

GJ $678.1 \mathrm{~A}$
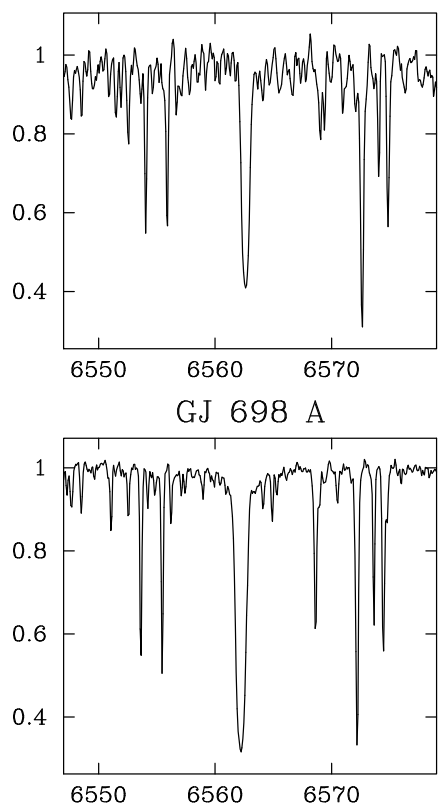

HD 234601

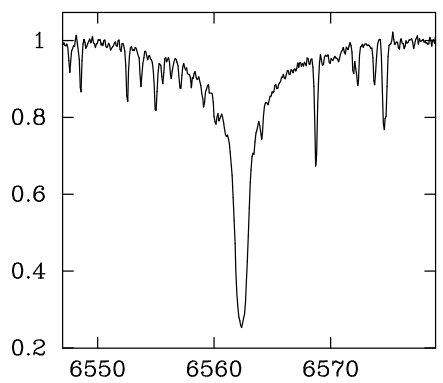

HD 171488

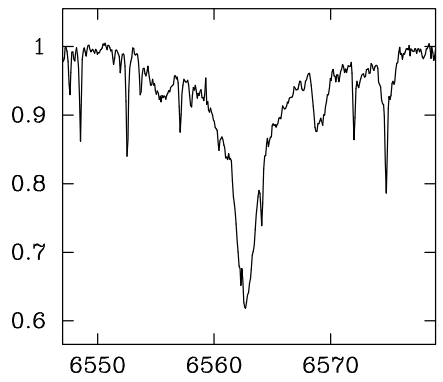

2RE J1846+191

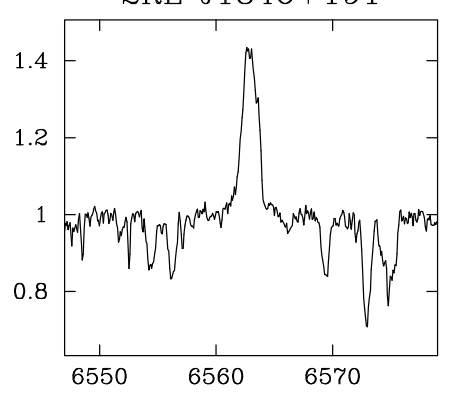

HD 160934
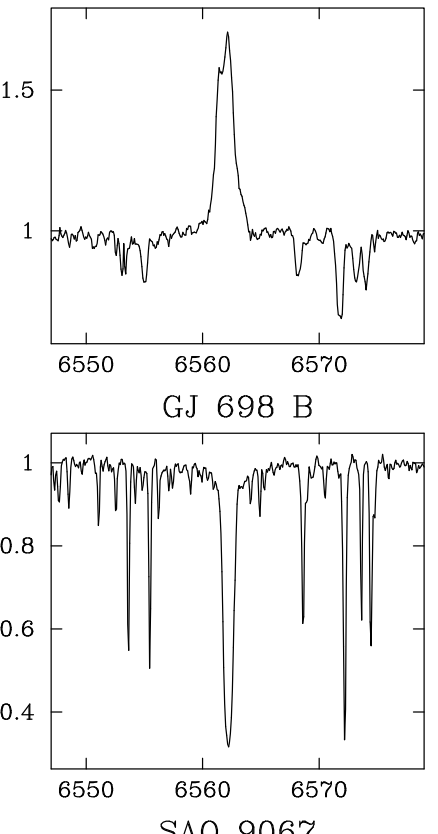

SAO 9067

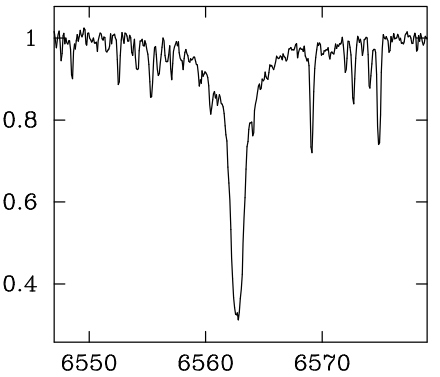

HD 171746

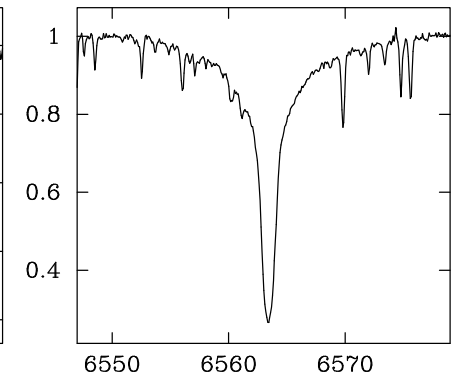

GJ 734 B

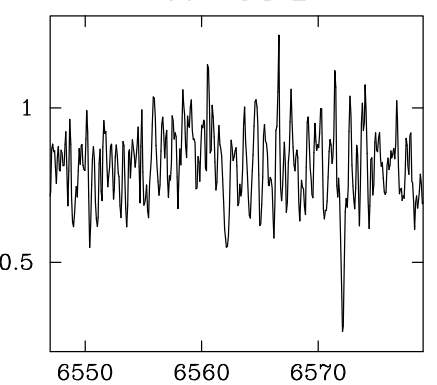


J. López-Santiago et al.: High-resolution spectroscopic survey of late-type stars
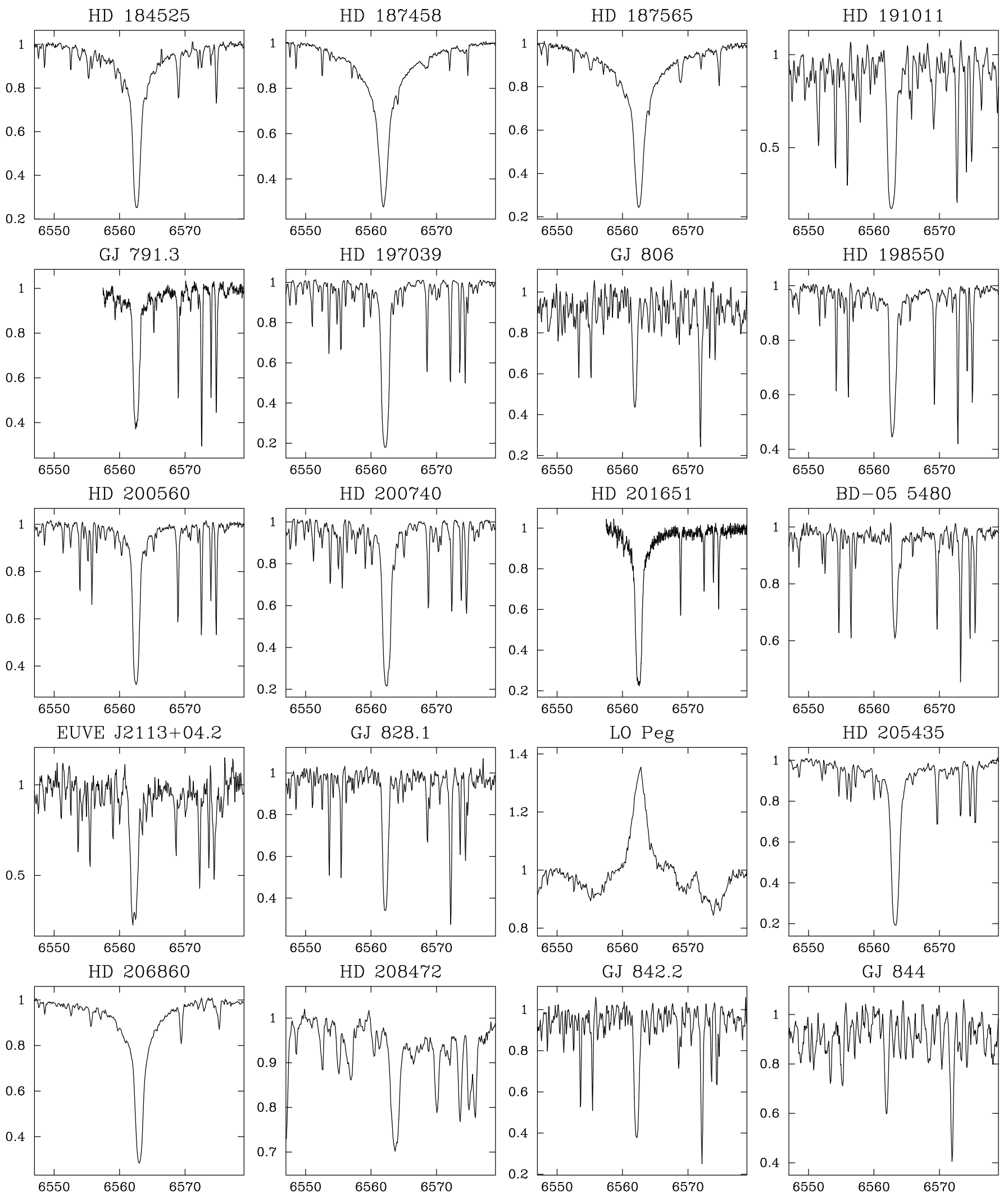

Fig. A.2. continued. 

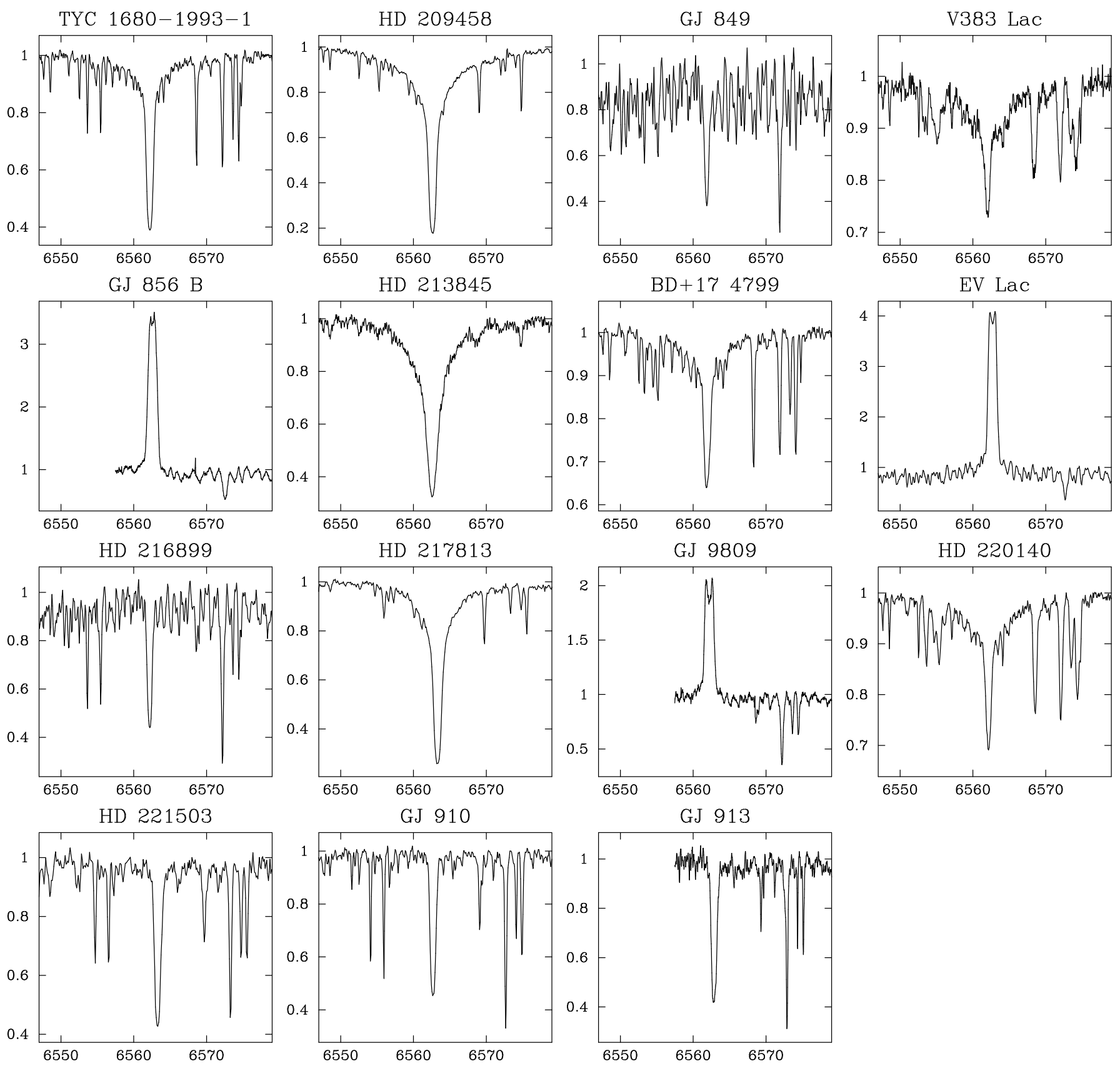

Fig. A.2. continued. 
J. López-Santiago et al.: High-resolution spectroscopic survey of late-type stars
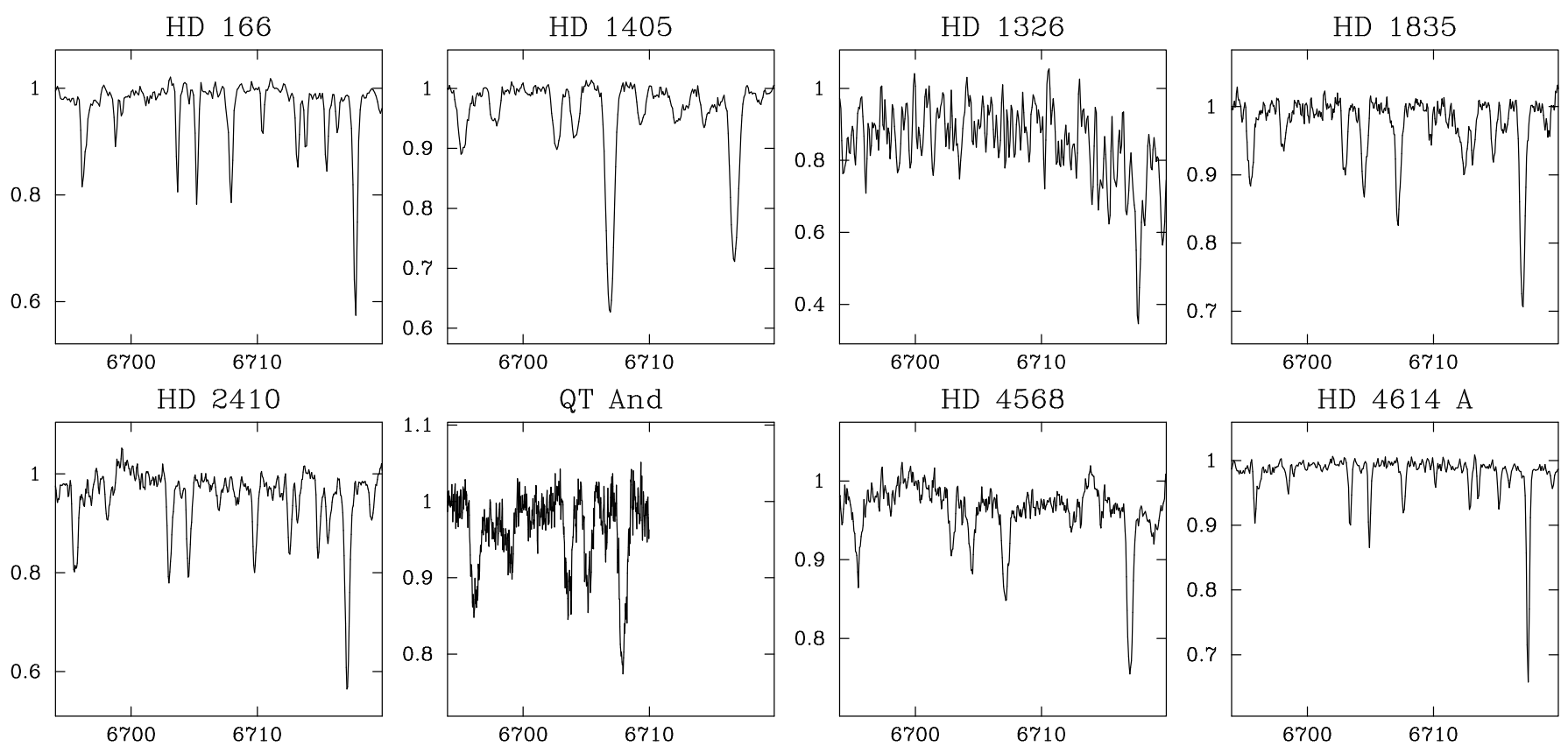

HD 4614 A

HD 4614 B

$\mathrm{BD}+17232$

HD 12230
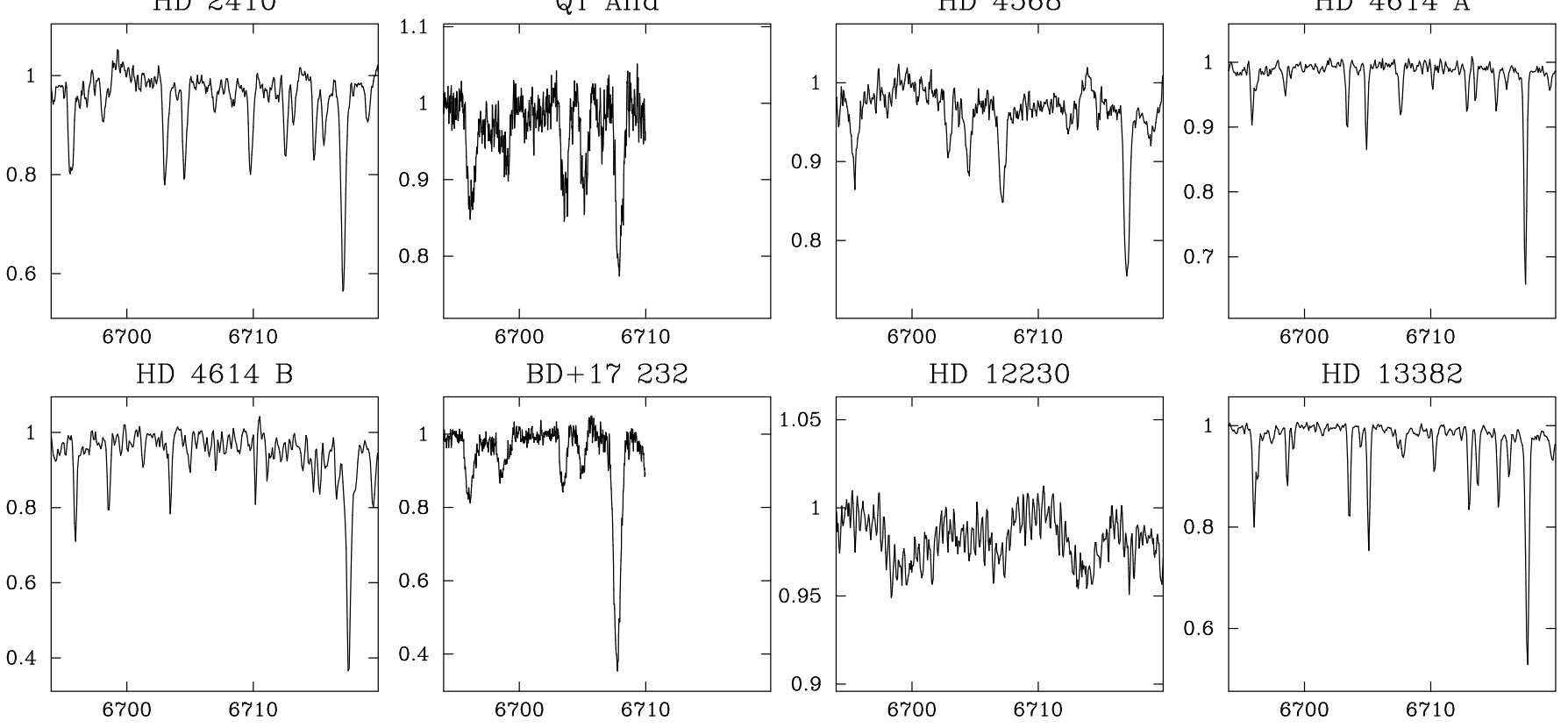

HD 13382
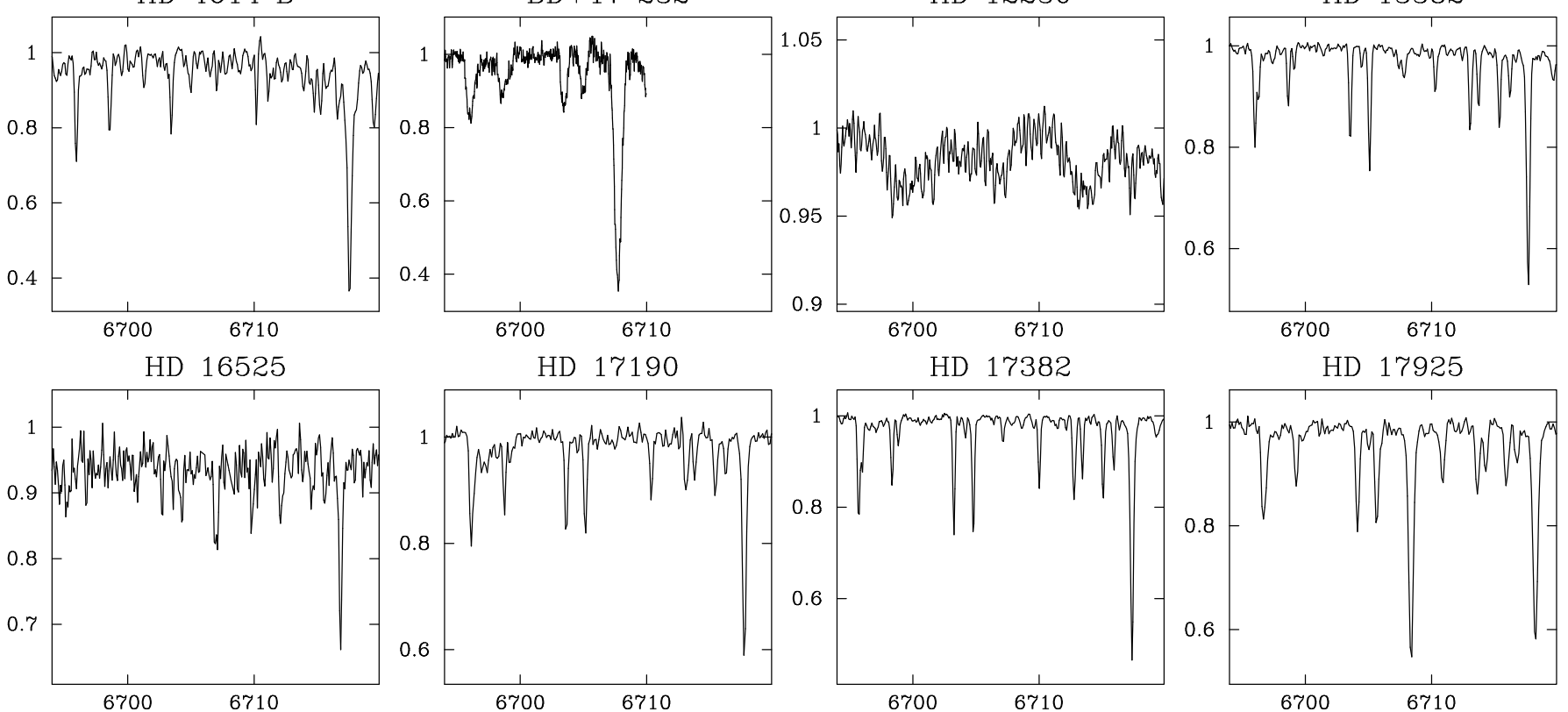

HD 17922

HD 18632
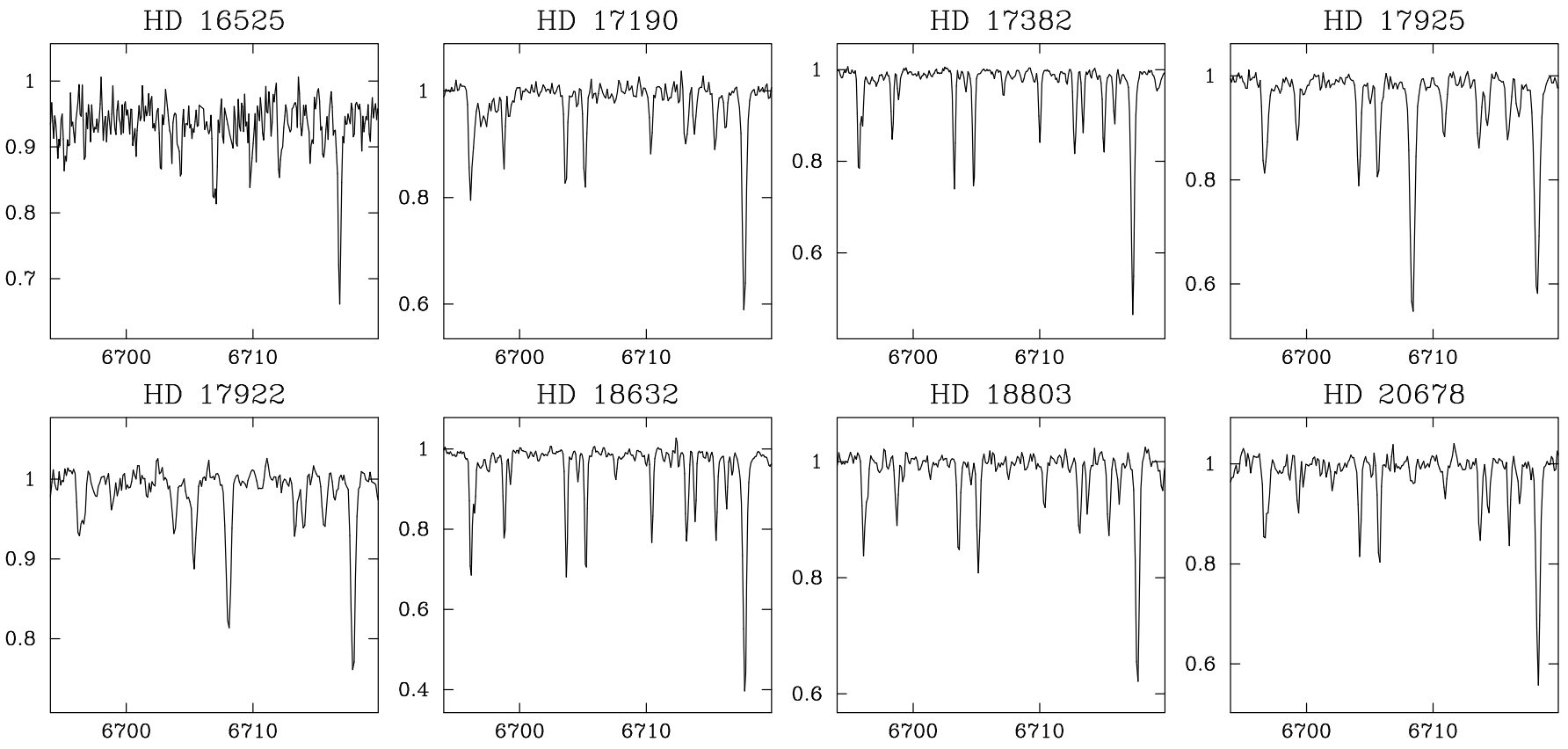

HD 20678

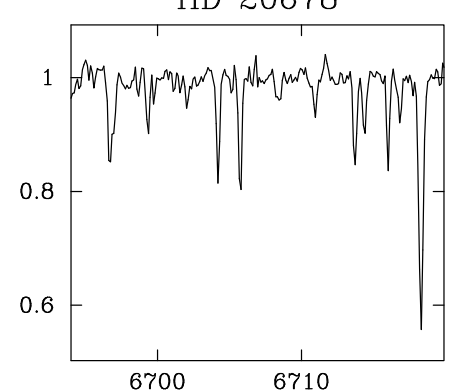

Fig. A.3. Li I spectra of the stars of our sample with observations of this line. 

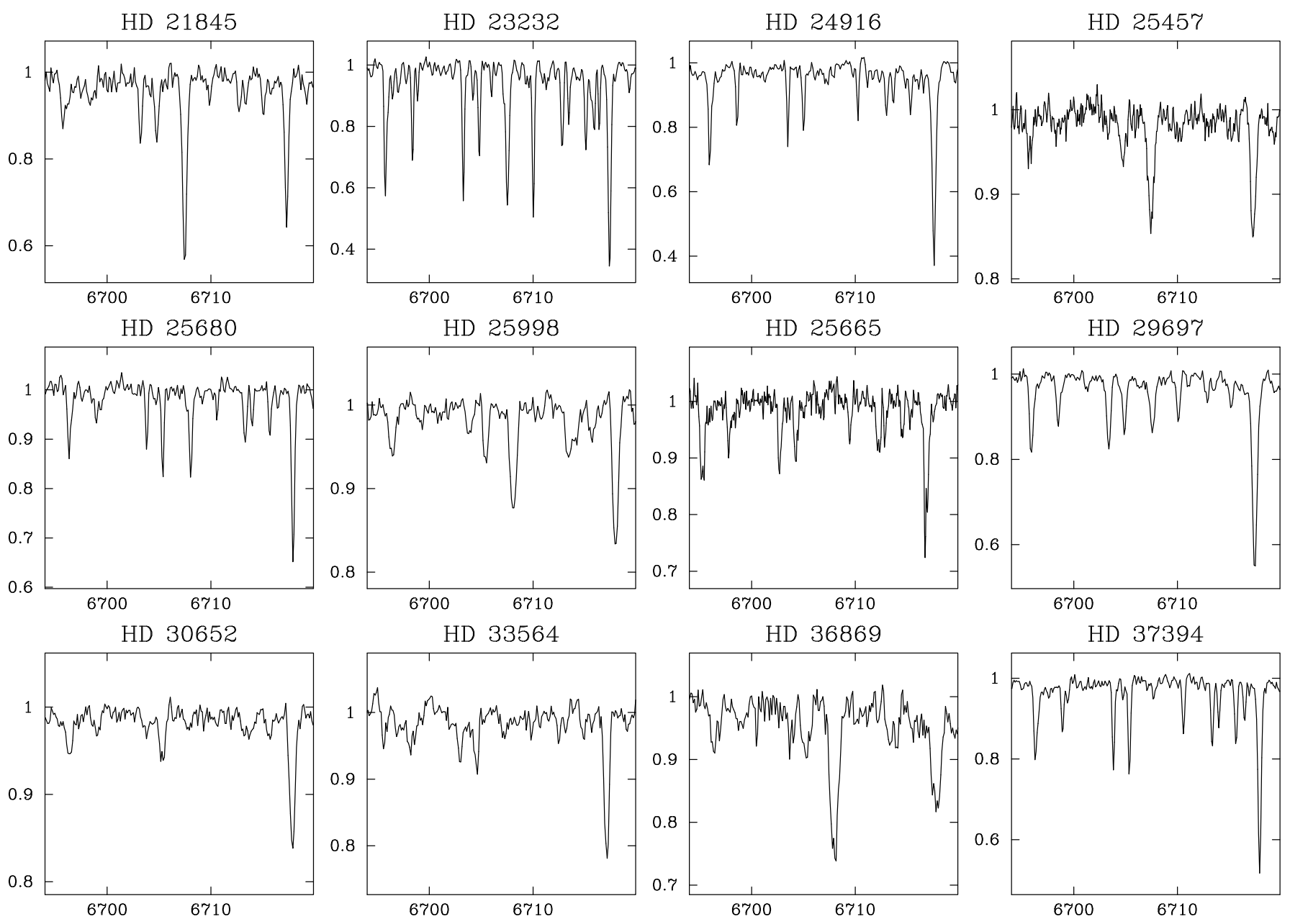

HD 37394
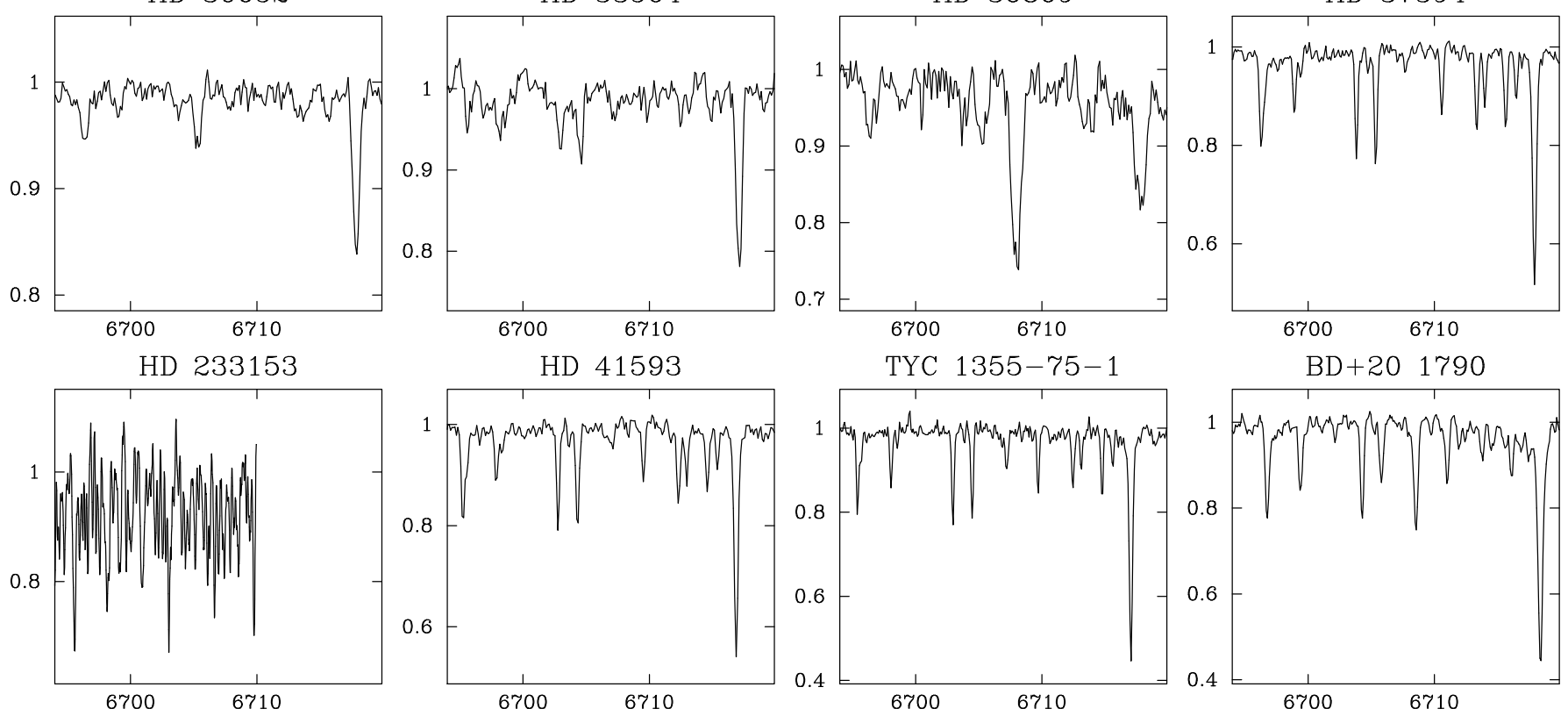

$\mathrm{BD}+071919$

$\mathrm{BD}+071919 \mathrm{~B}$

FP Cnc
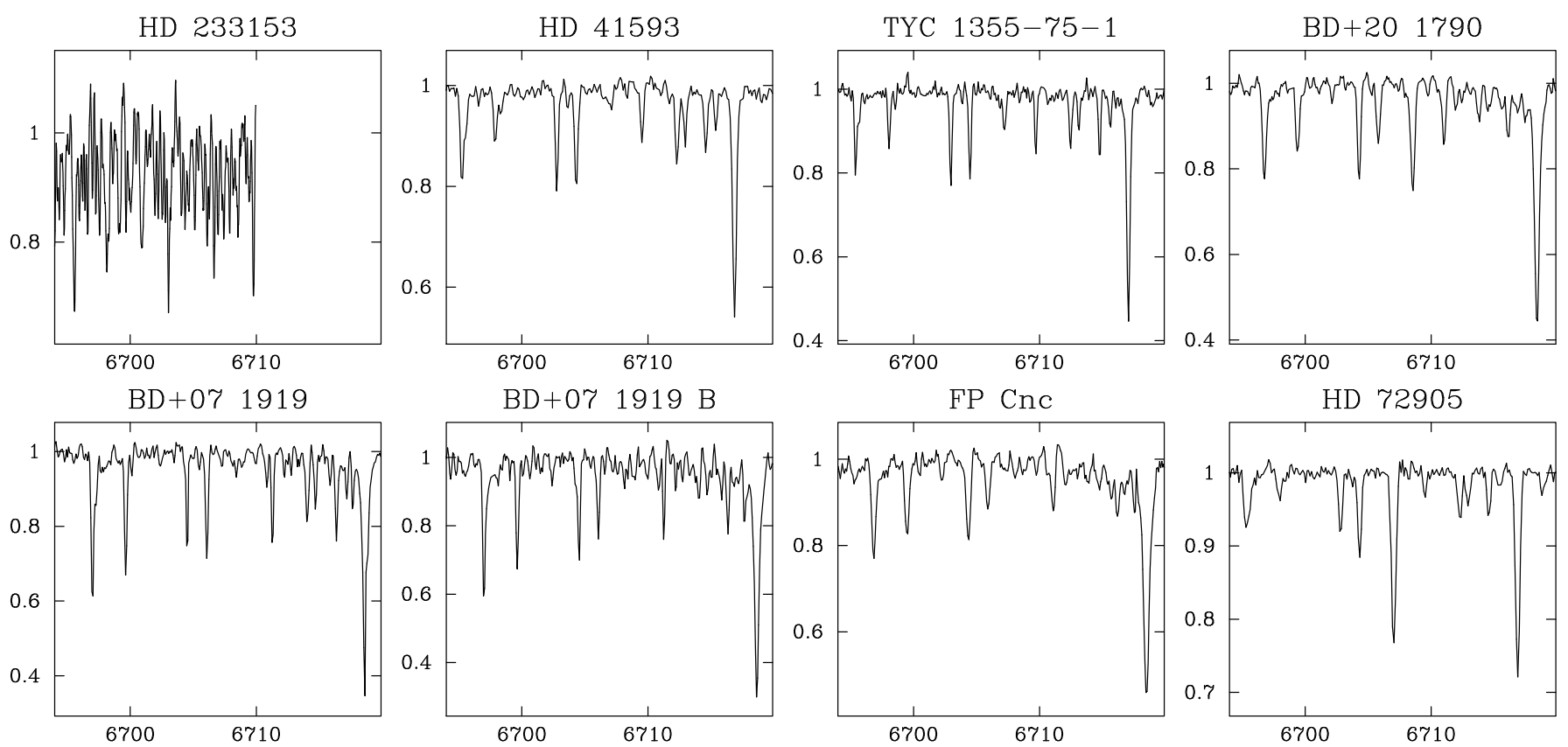

Fig. A.3. continued. 
J. López-Santiago et al.: High-resolution spectroscopic survey of late-type stars
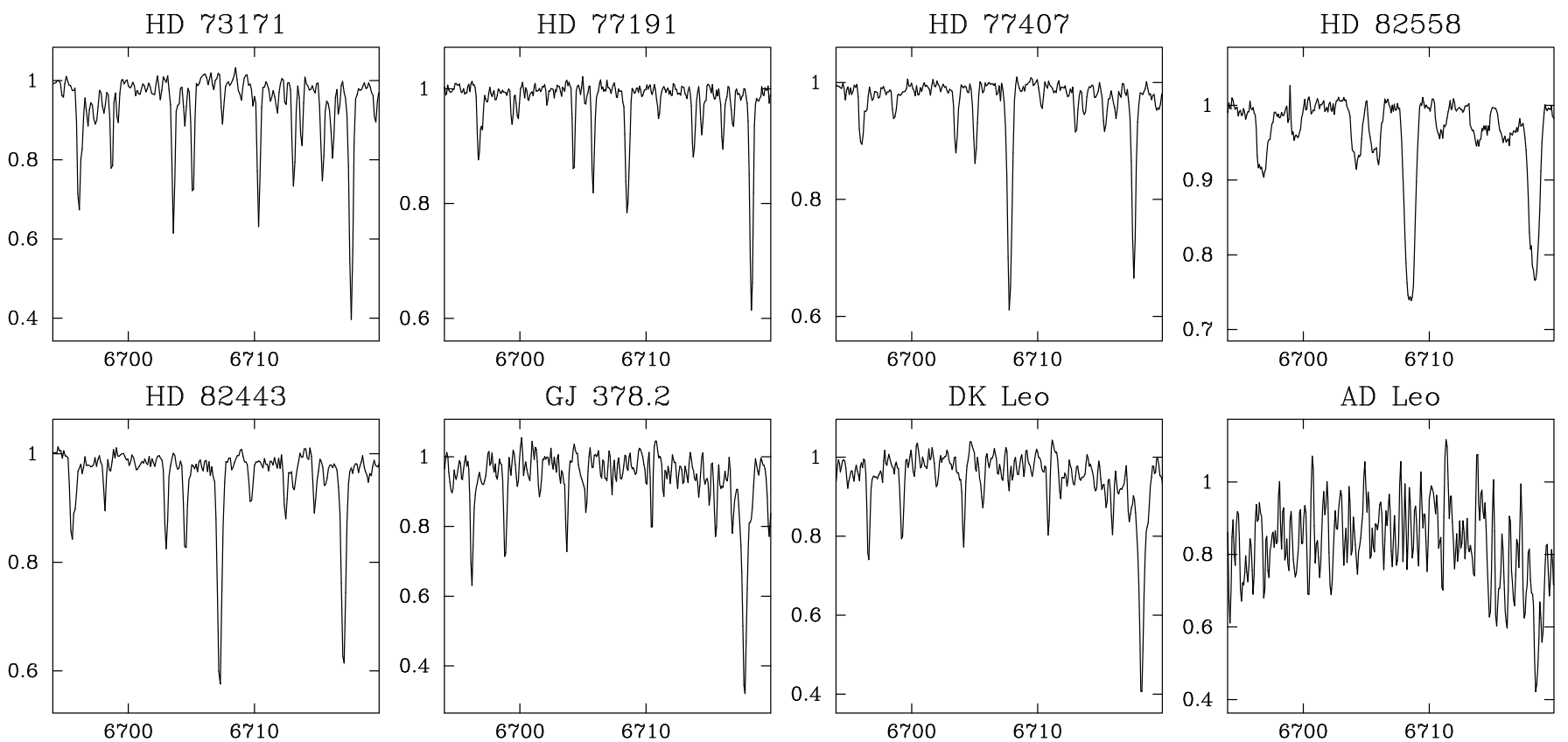

HD 85270
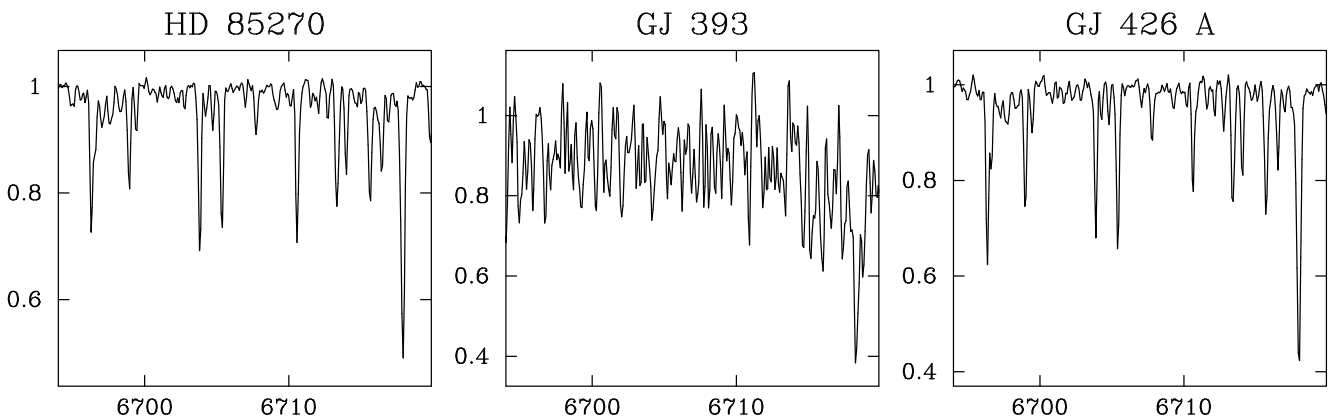

GJ 426 B
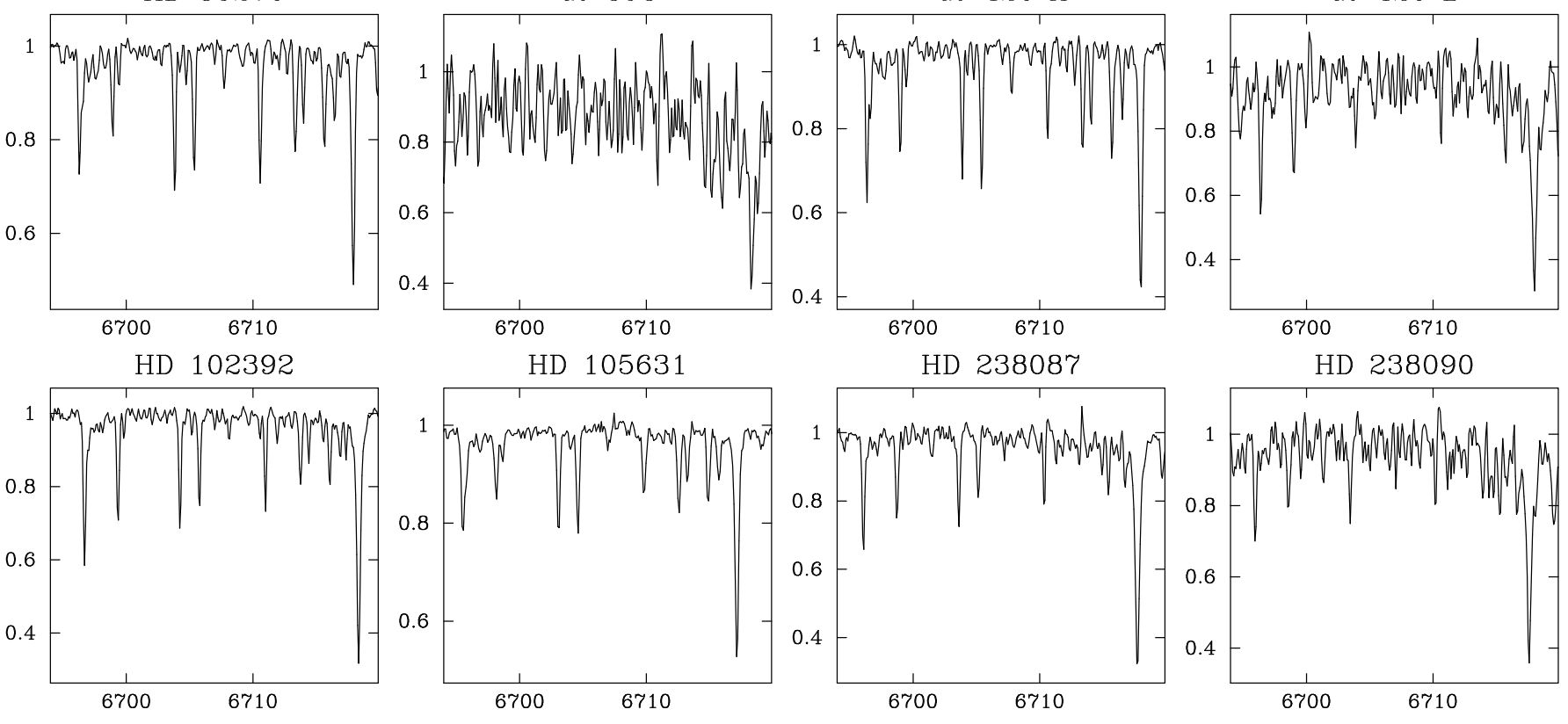

HD 106496
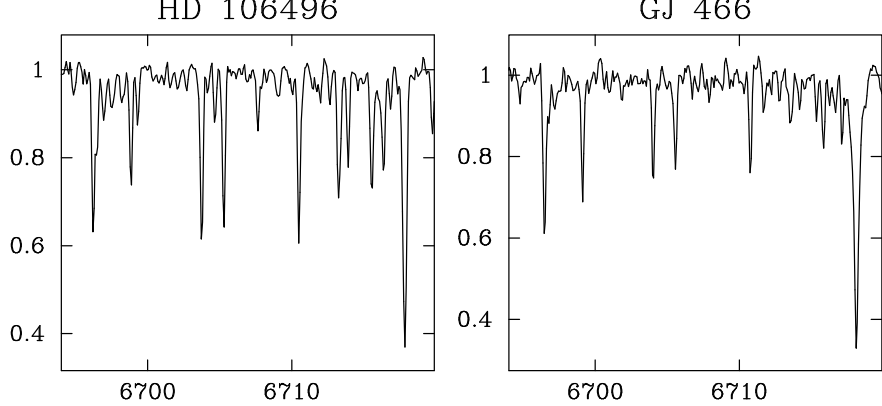

HD 110010
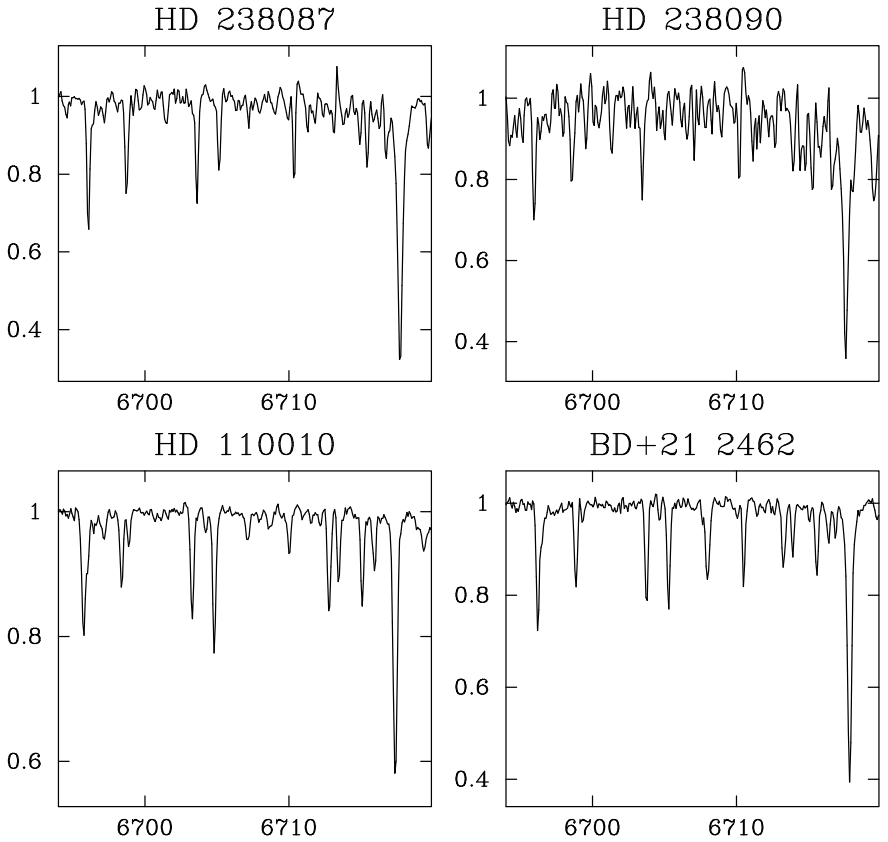

Fig. A.3. continued. 
A\&A 514, A97 (2010)
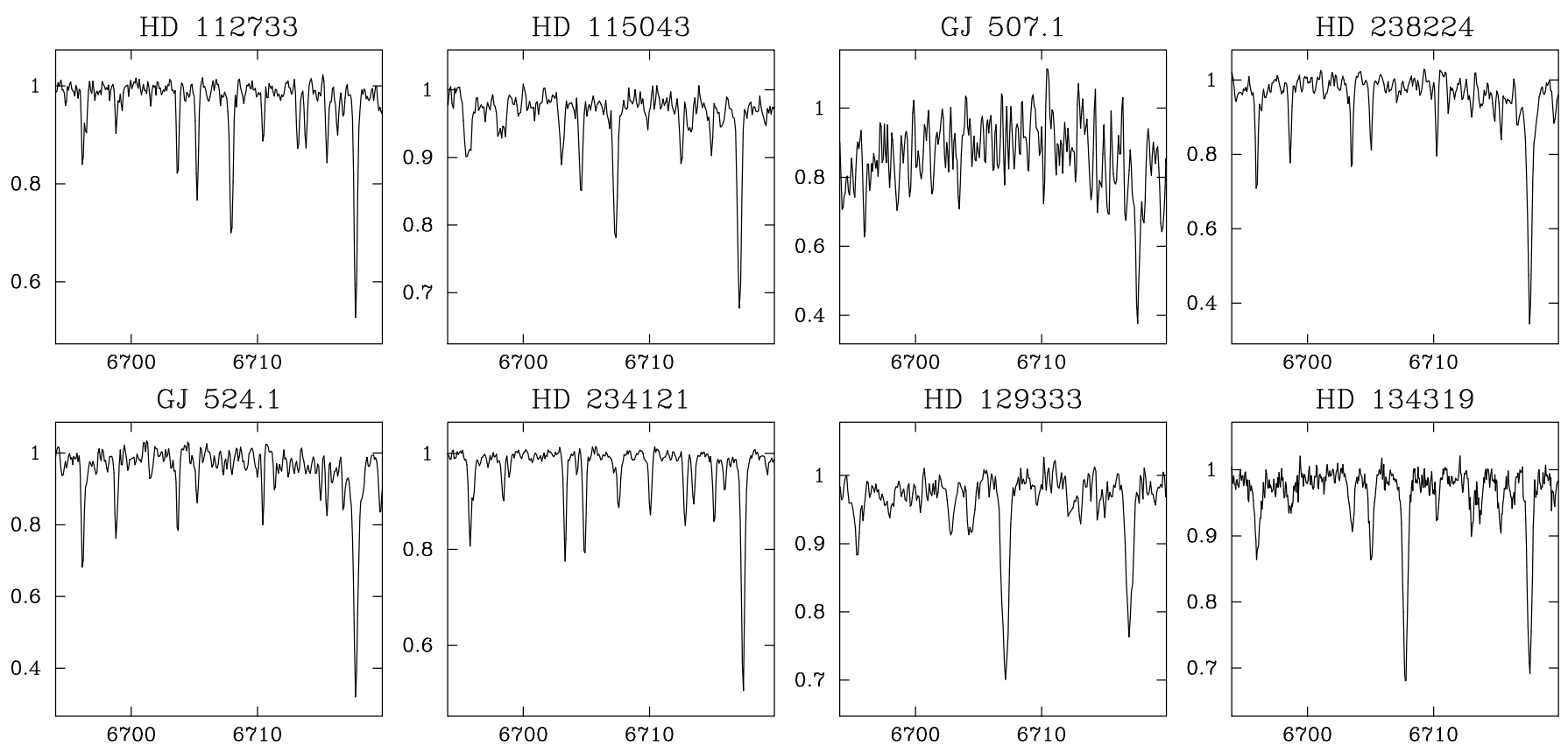

HD 234121

HD 129333

HD 134319
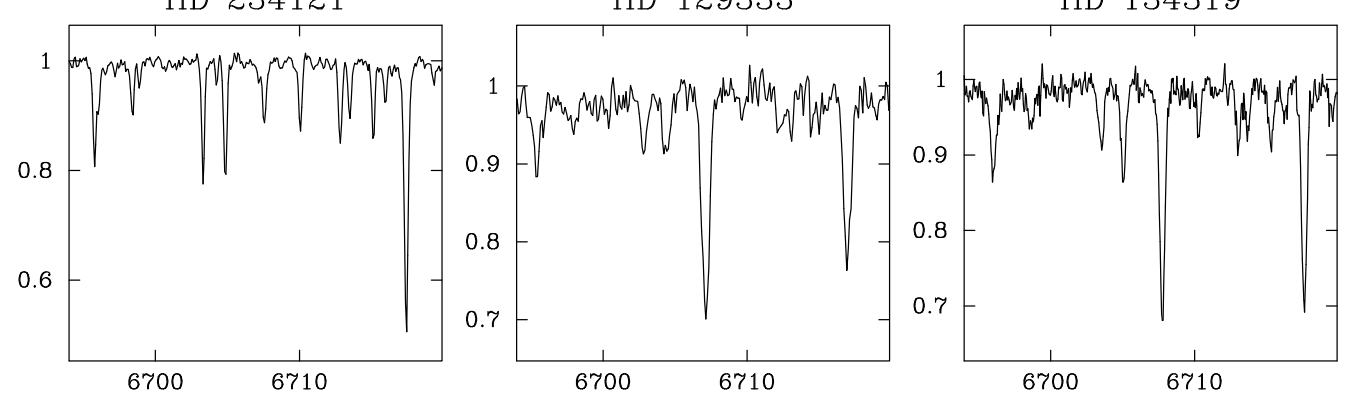

HD 135363

HD 140913

HD 142764

HD 143809
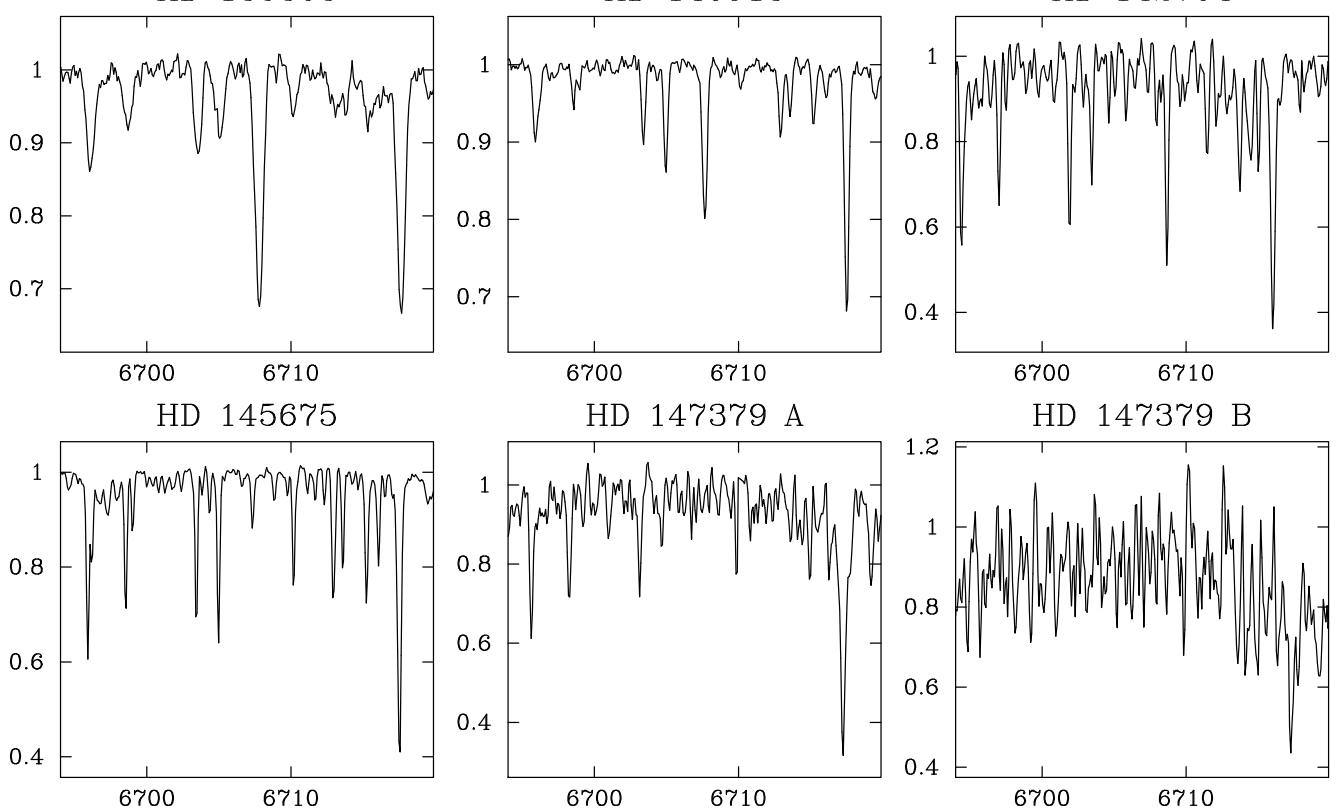

HD 147379 A

HD 147379 B
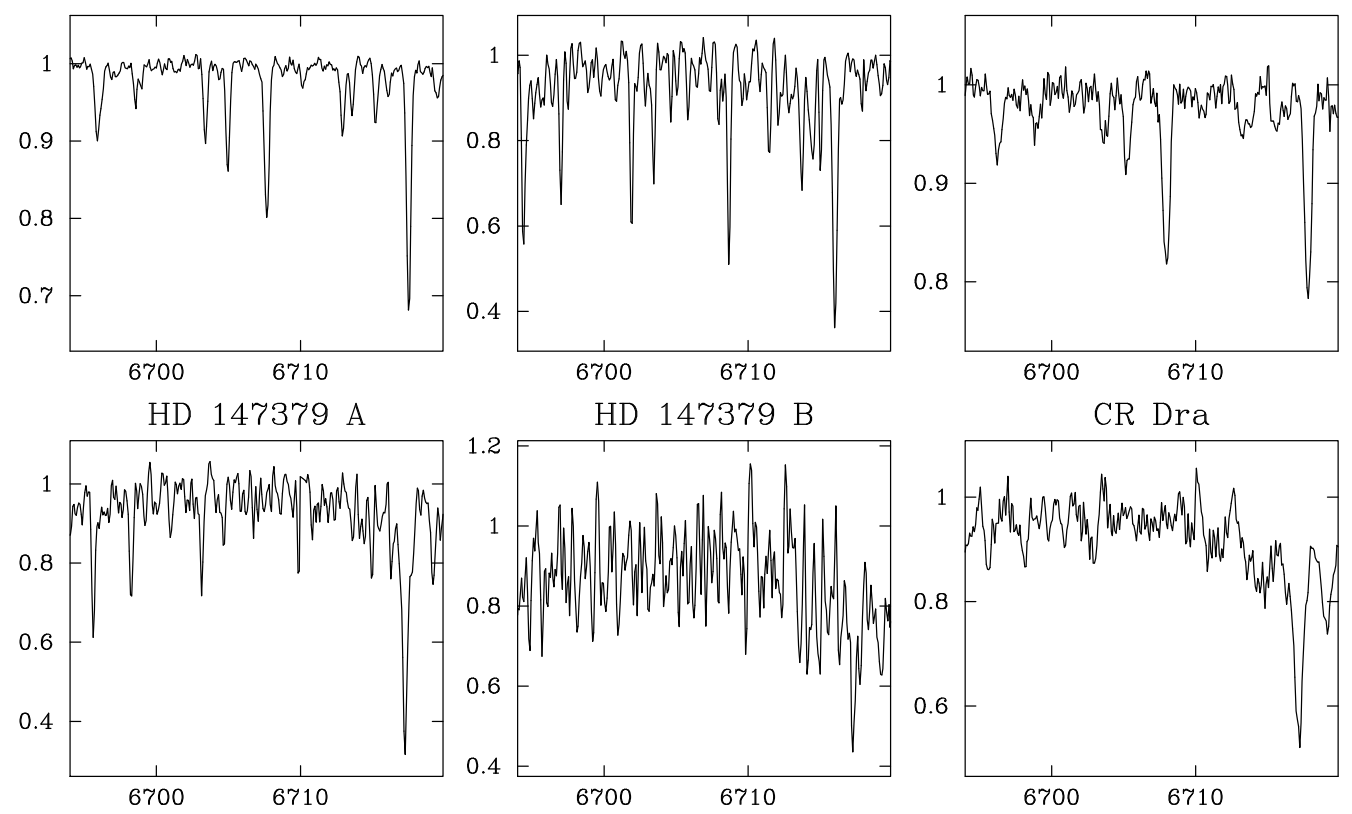

HD 149661
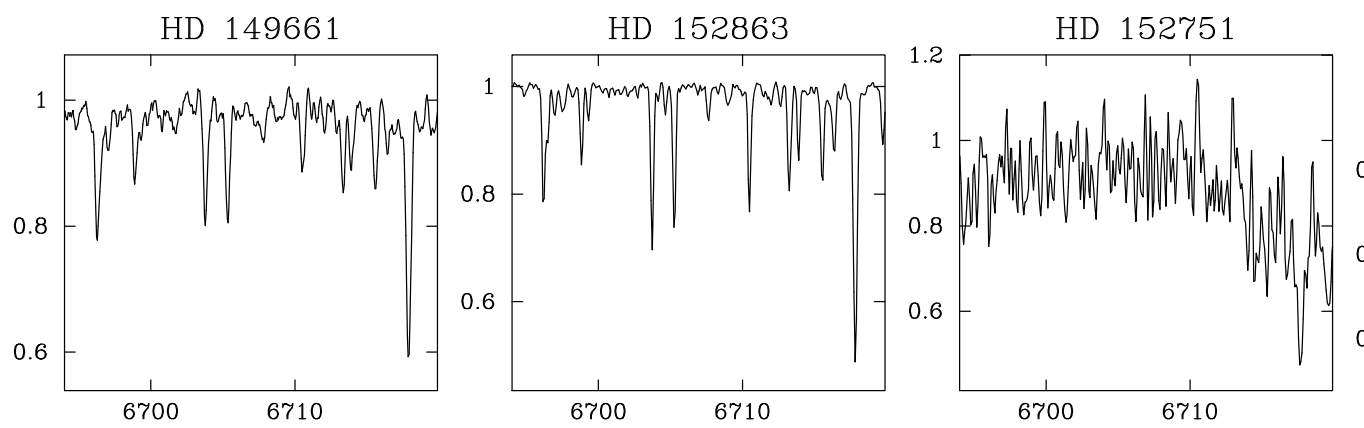

HD 155674 A

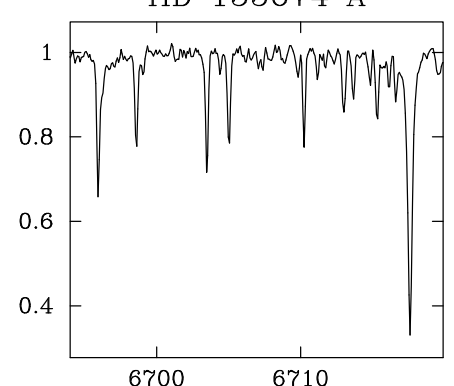

Fig. A.3. continued. 
J. López-Santiago et al.: High-resolution spectroscopic survey of late-type stars
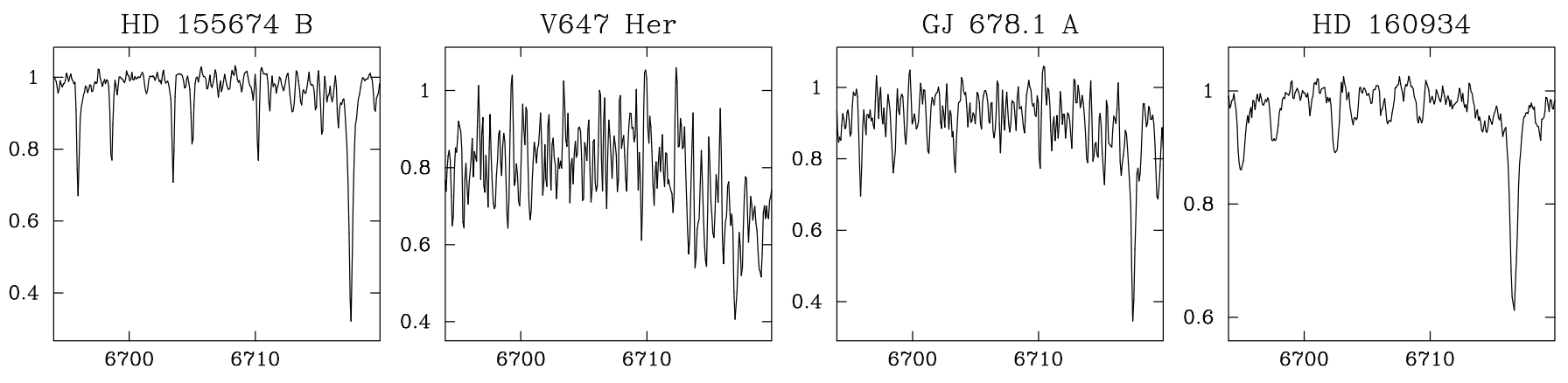

HD 162283
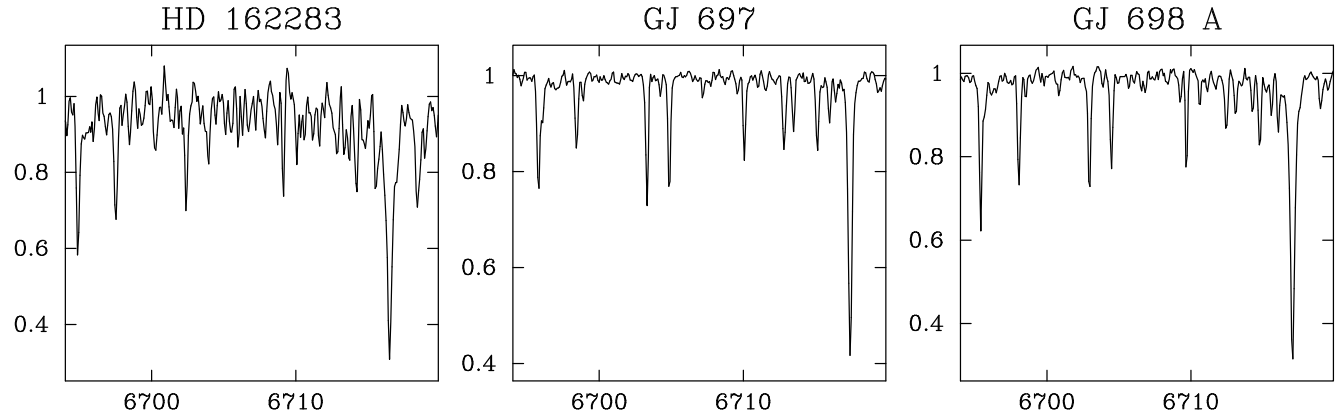

GJ 698 B

HD 165341

HD 167605

HD 234601
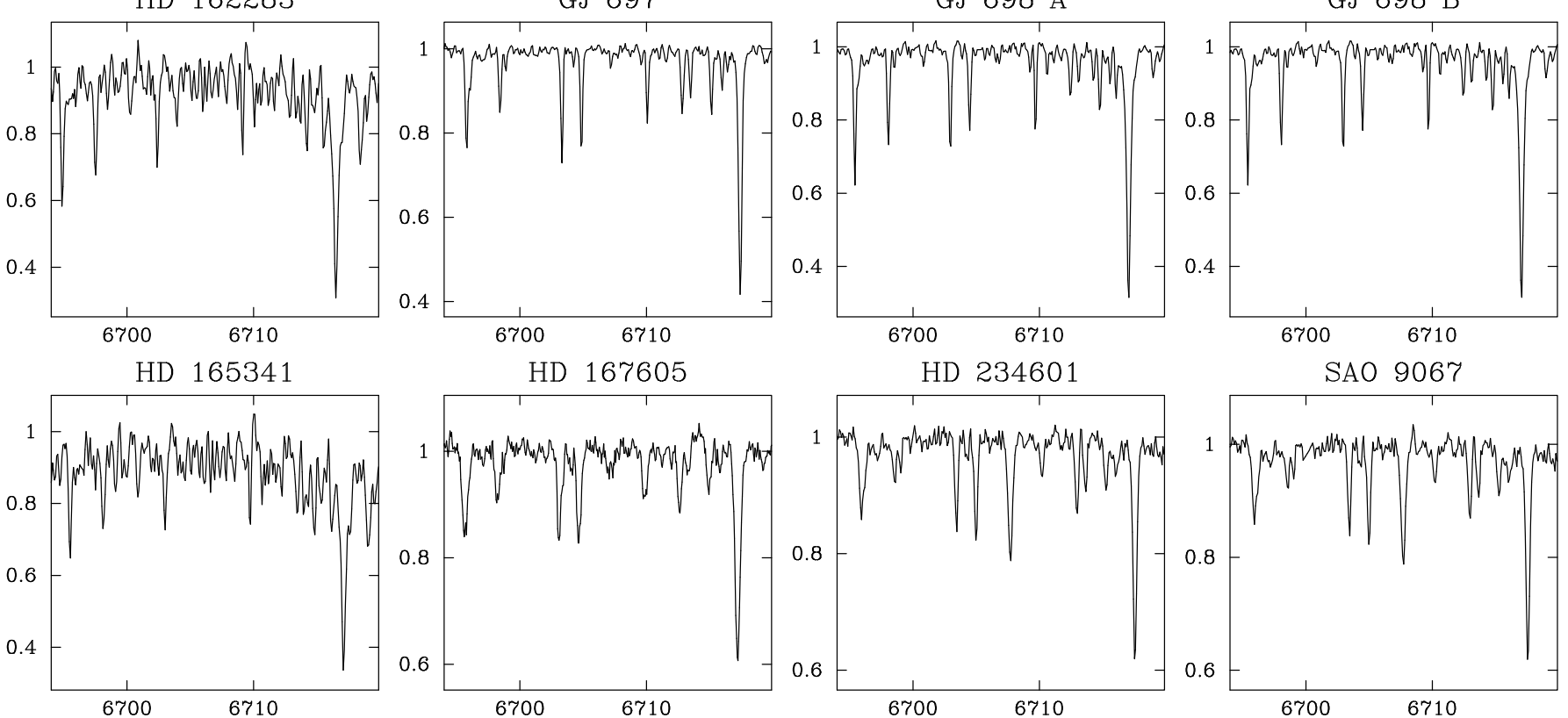

SAO 9067

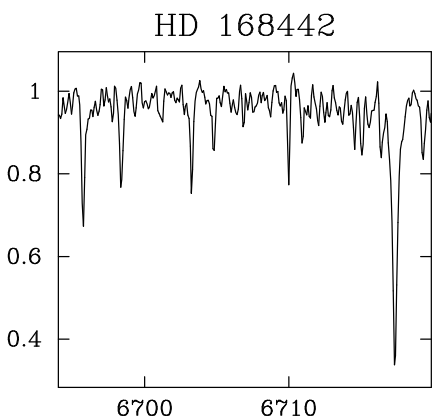

FK Ser
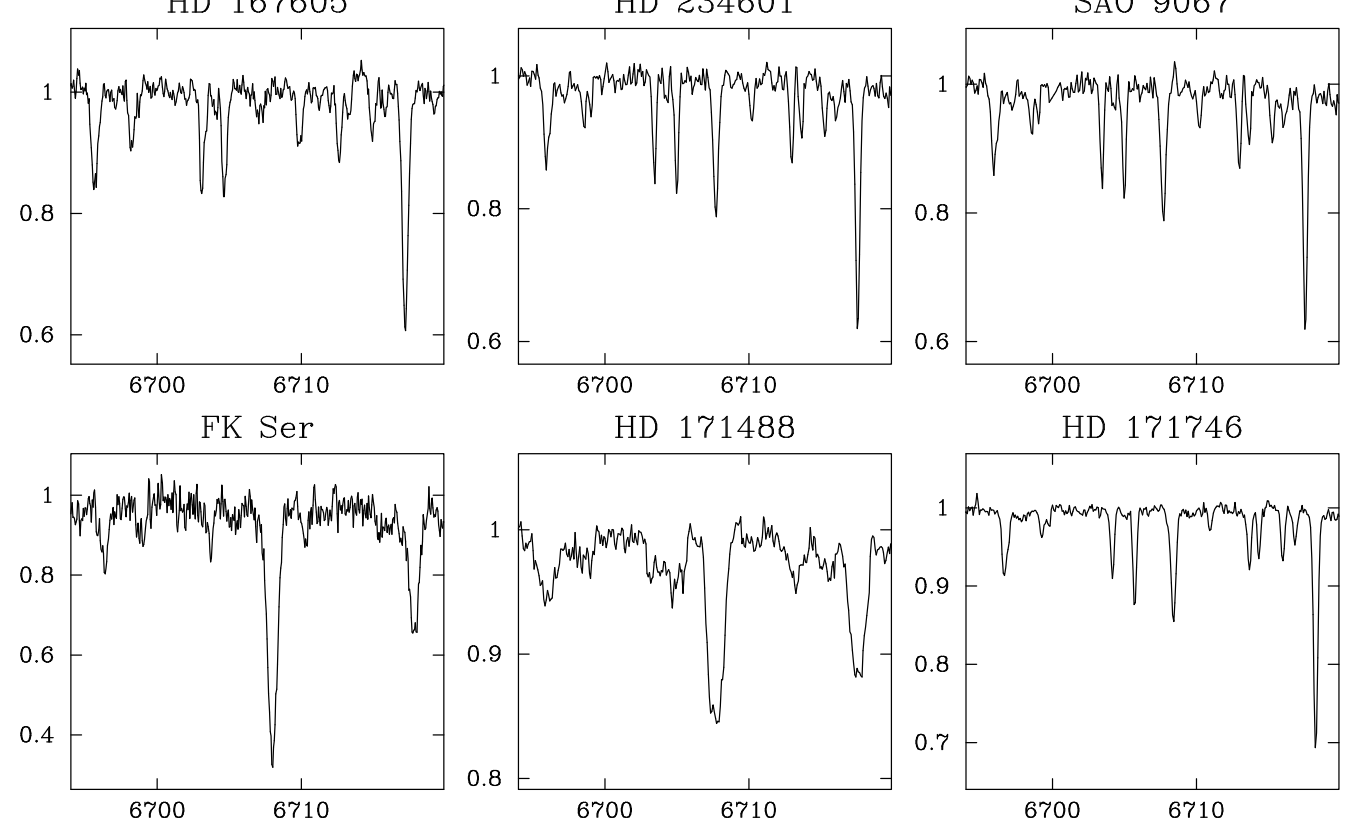

HD 173739

HD 173740

2RE J1846+191
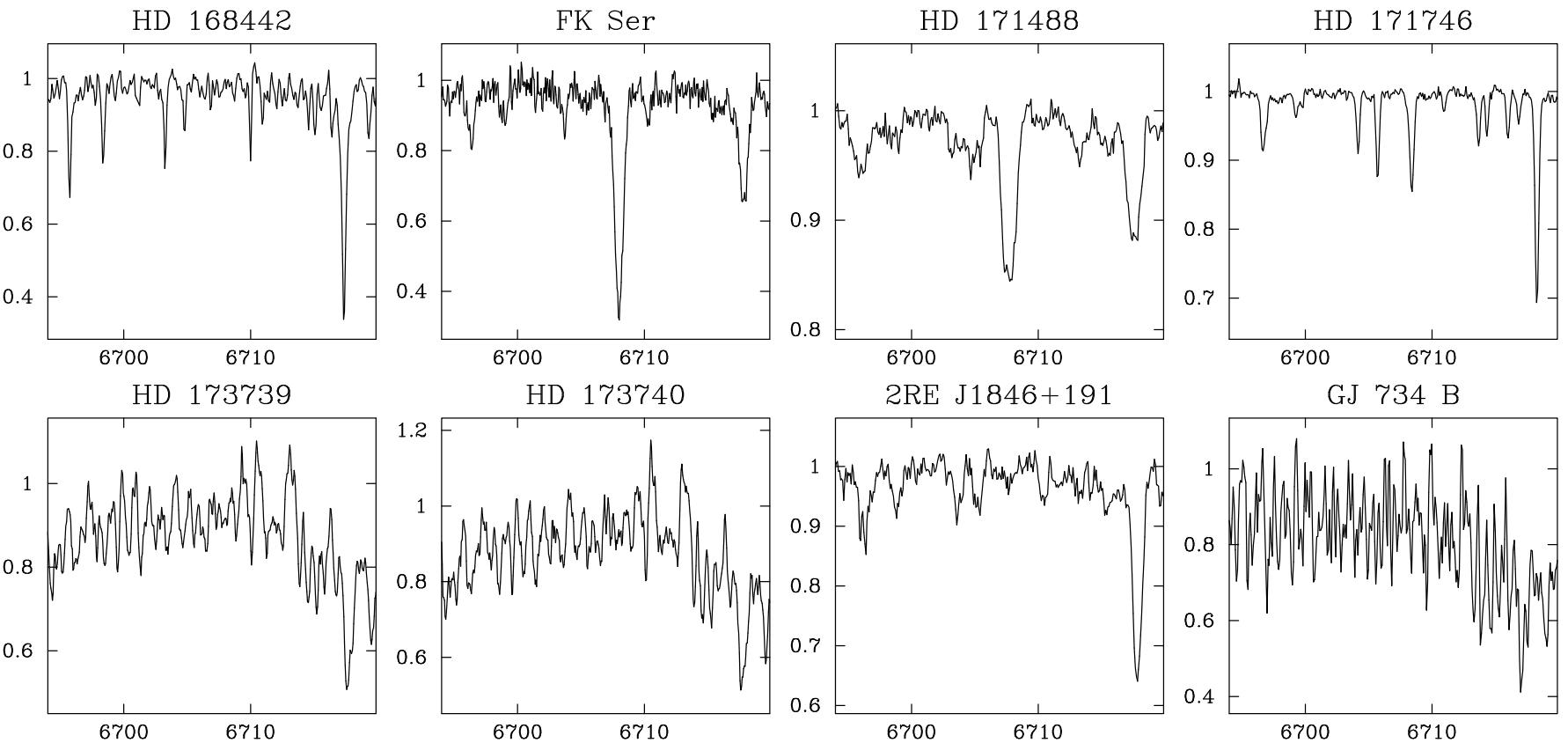

GJ 734 B

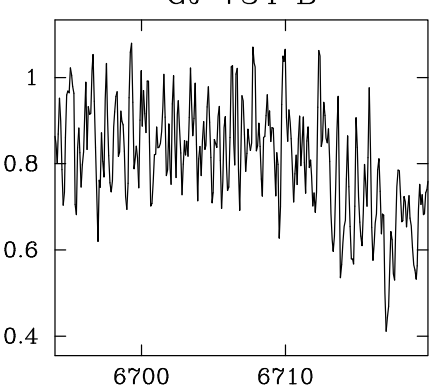

Fig. A.3. continued. 
A\&A 514, A97 (2010)
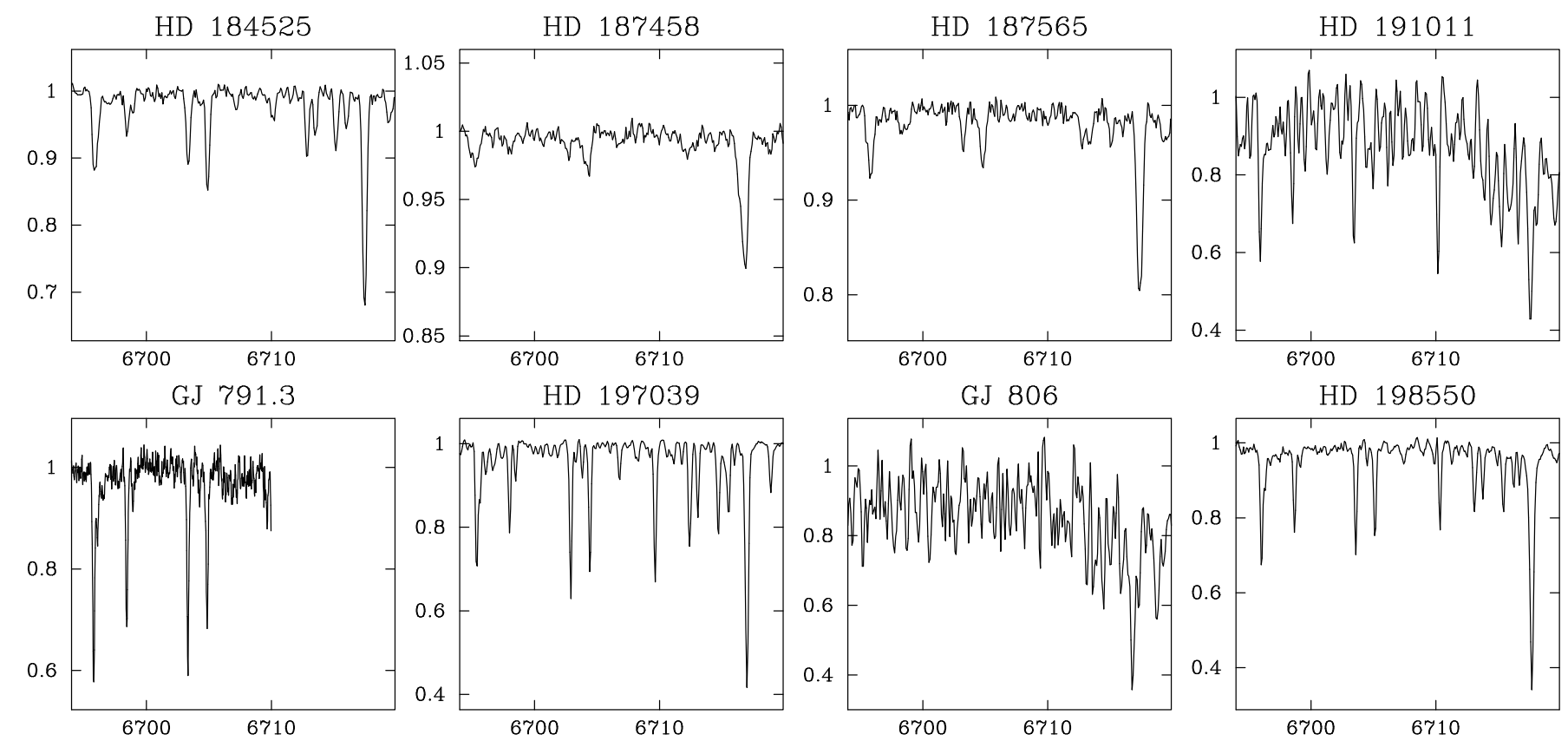

HD 198550
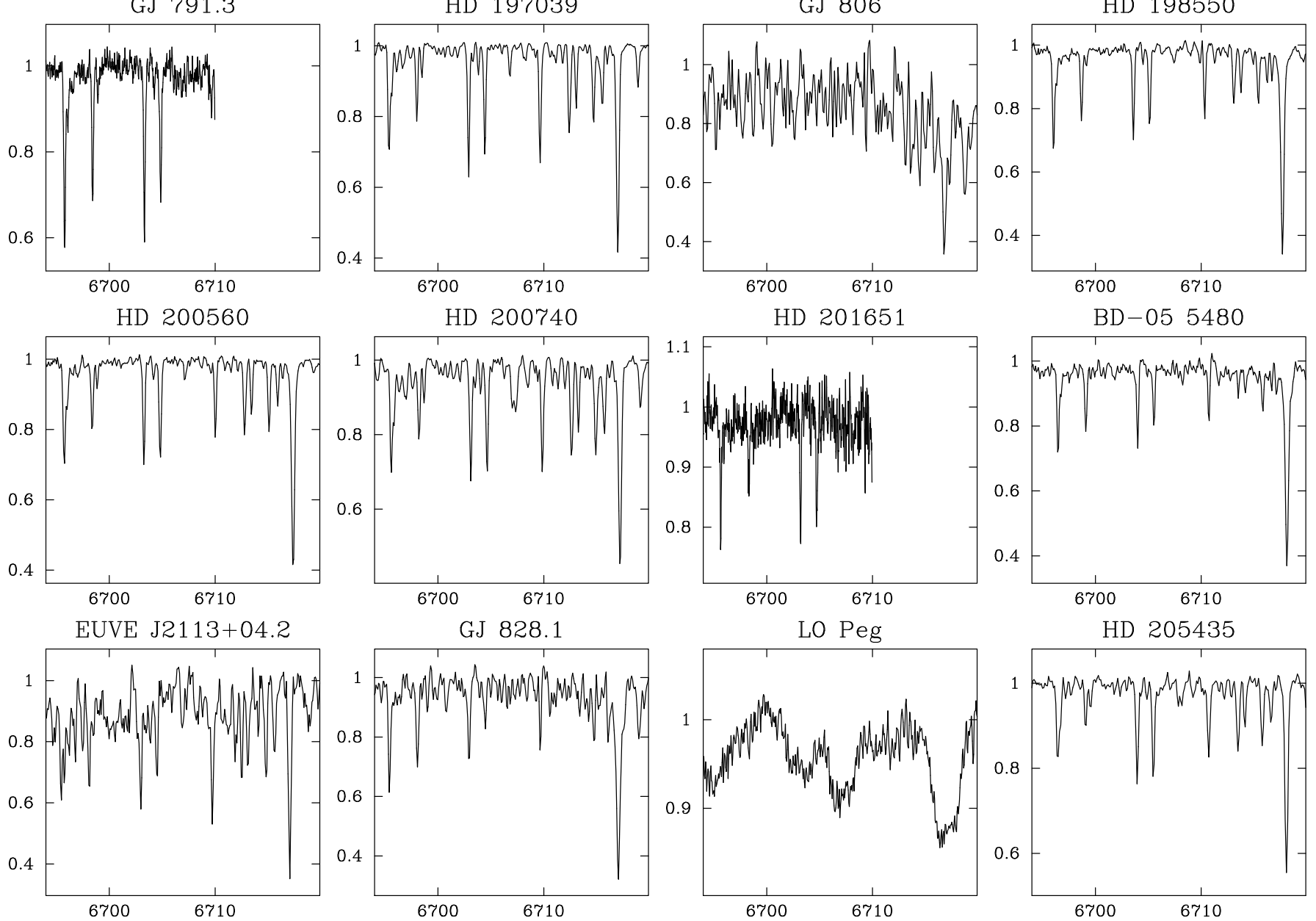

HD 206860

HD 208472

GJ 842.2
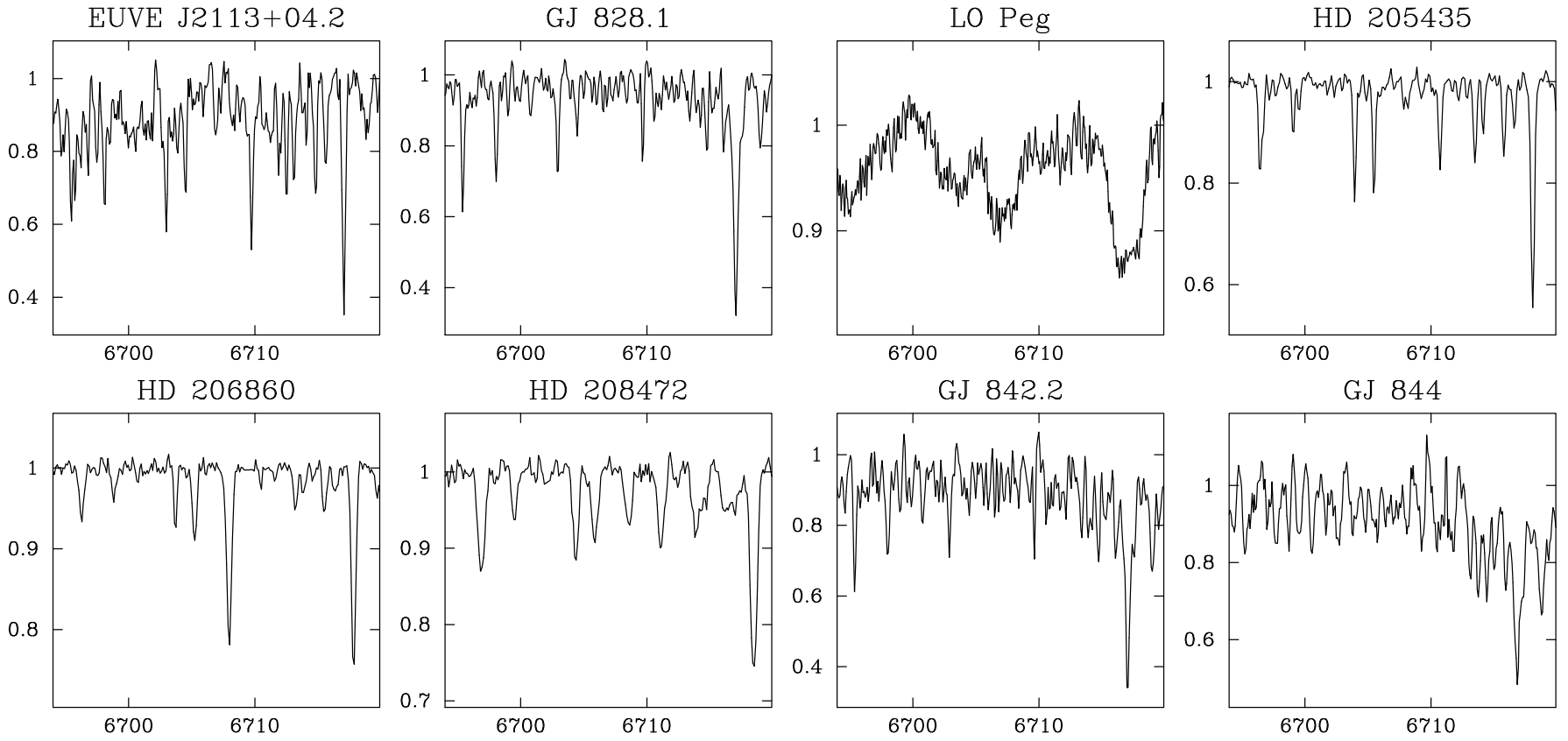

GJ 844

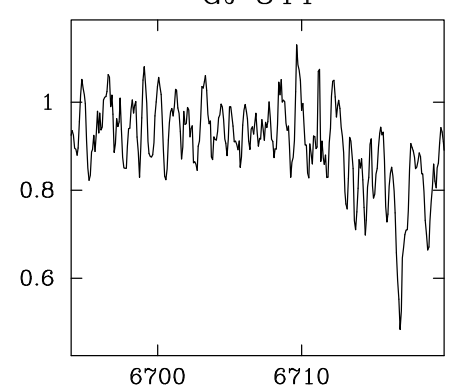

Fig. A.3. continued. 
J. López-Santiago et al.: High-resolution spectroscopic survey of late-type stars
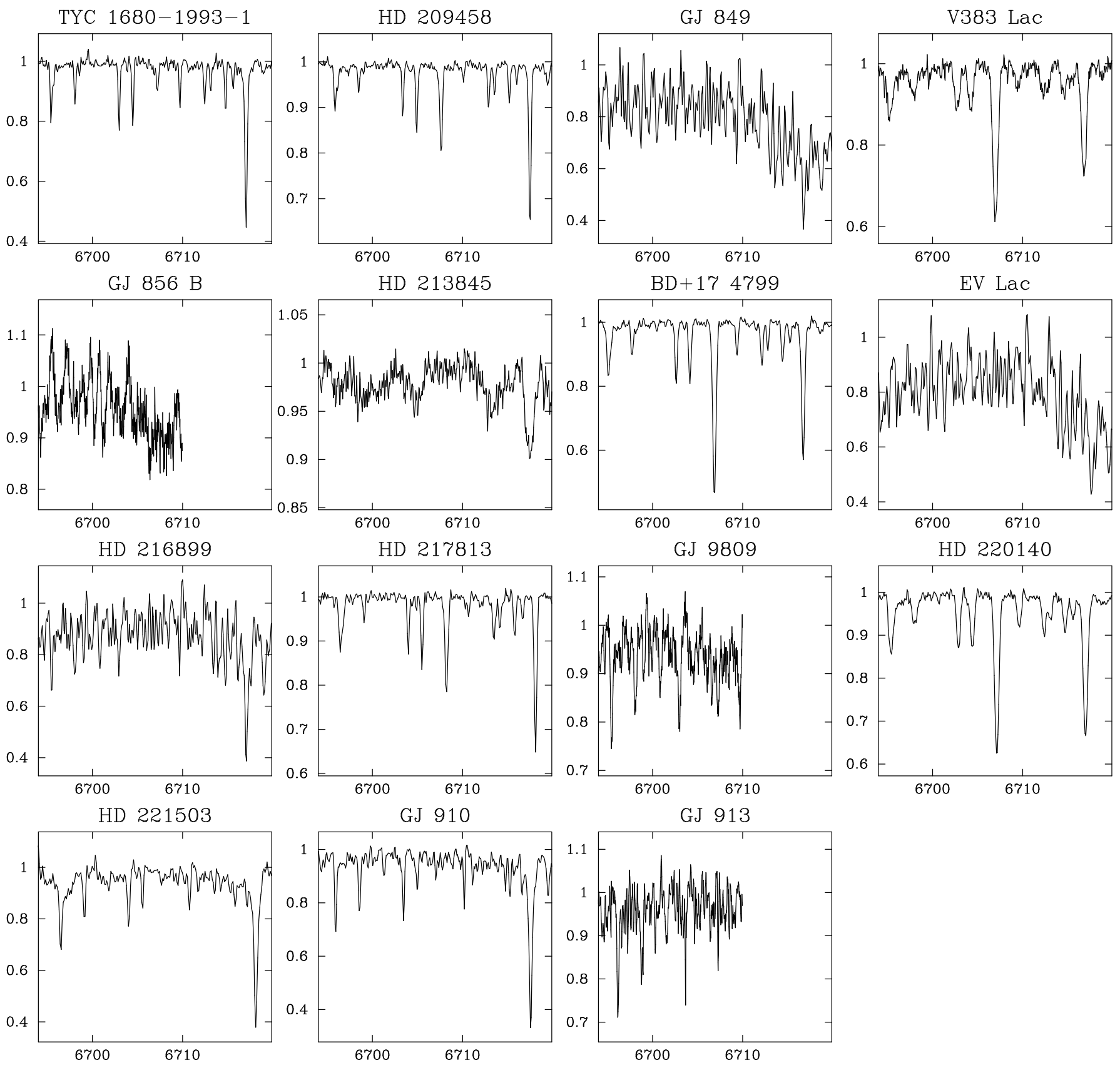

Fig. A.3. continued. 

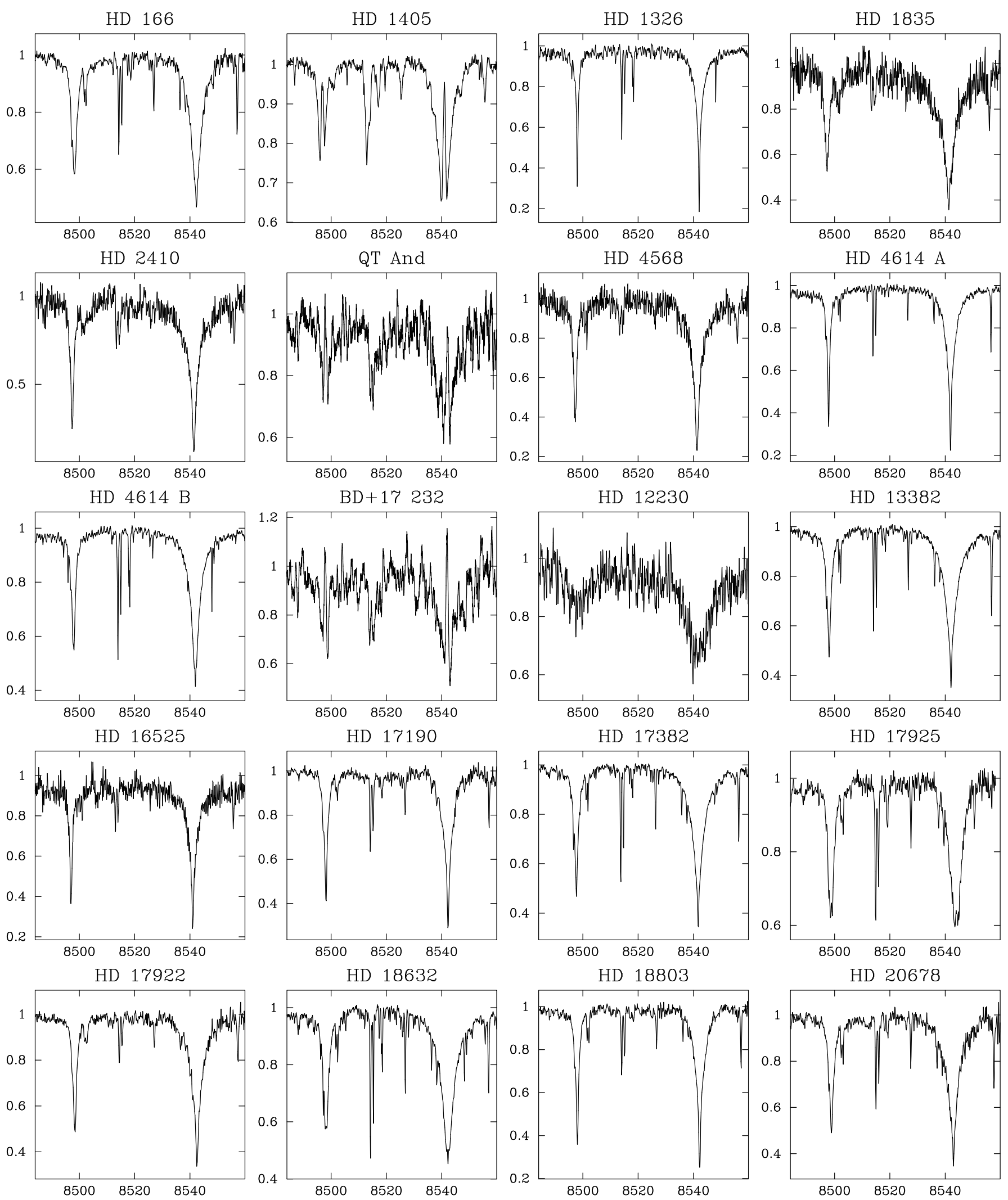

Fig. A.4. Ca II $\lambda \lambda 8498$ and $8540 \AA$ spectra of the stars of our sample with observations of the Ca II infrared triplet. 
J. López-Santiago et al.: High-resolution spectroscopic survey of late-type stars

HD 21845
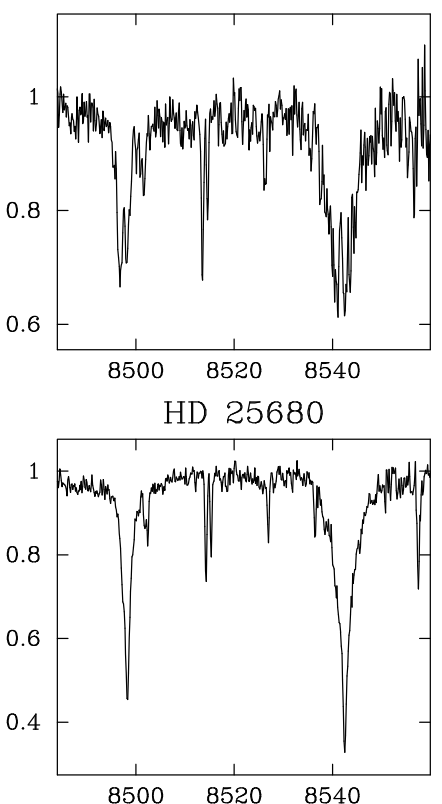

HD 30652

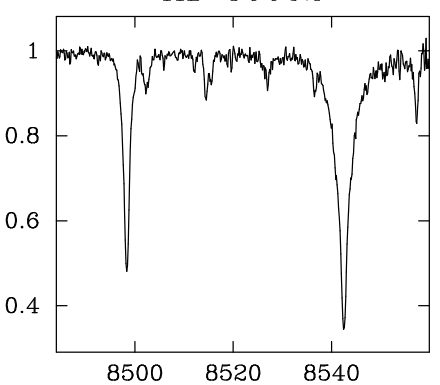

HD 233153

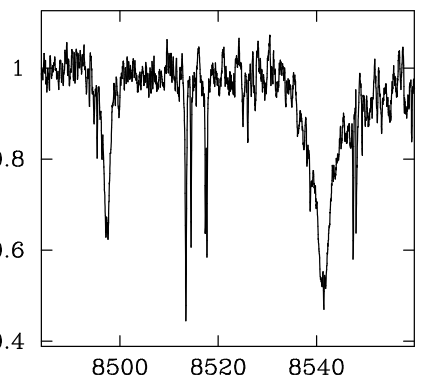

$\mathrm{BD}+071919$

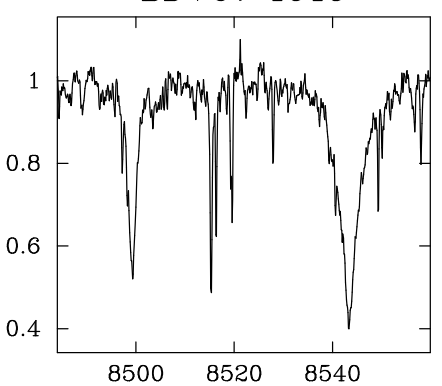

Fig. A.4. continued.
HD 23232

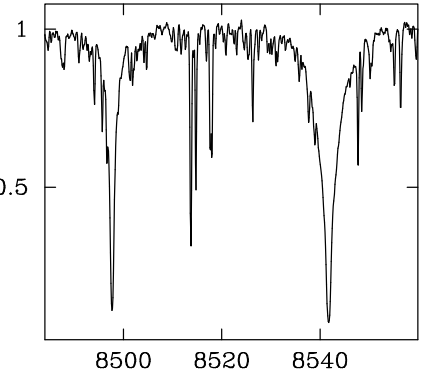

HD 25998

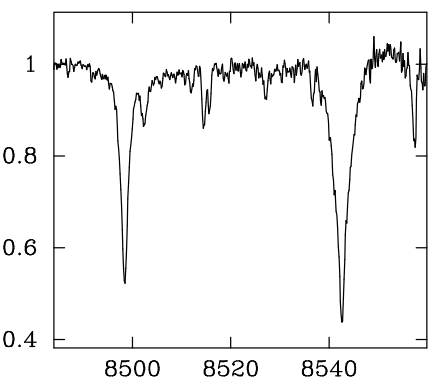

HD 33564

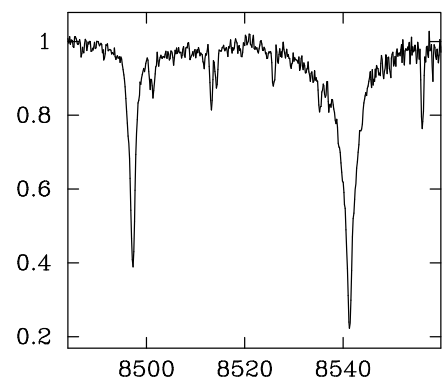

HD 41593

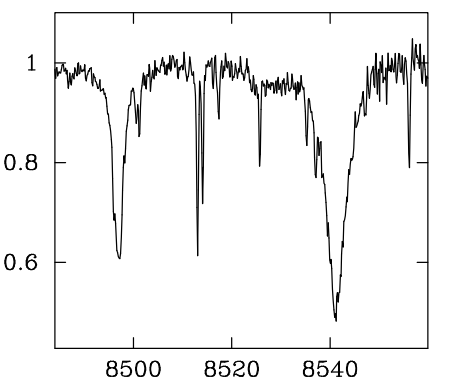

$\mathrm{BD}+071919 \mathrm{~B}$

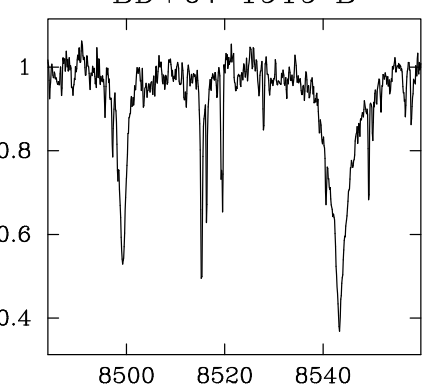

HD 24916

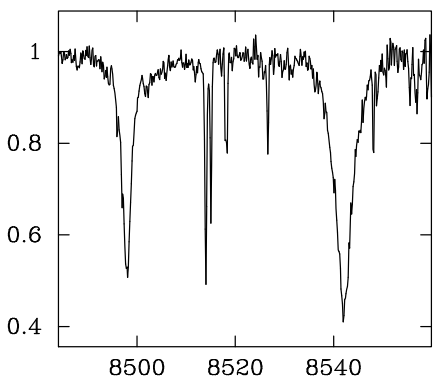

HD 25665

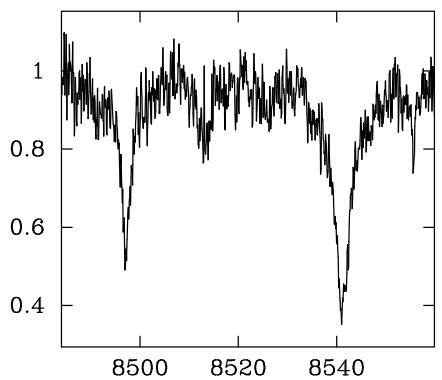

HD 36869

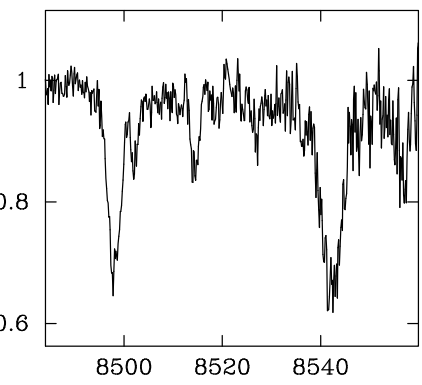

TYC $1355-75-1$

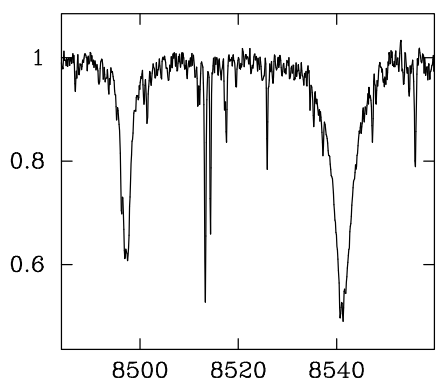

FP Cnc

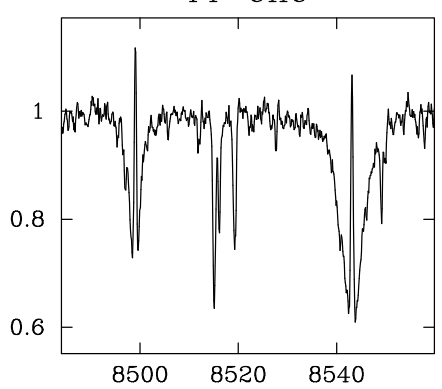

HD 25457

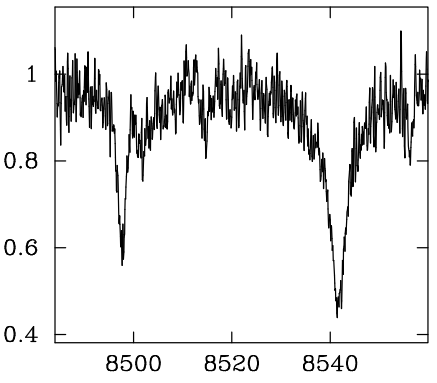

HD 29697

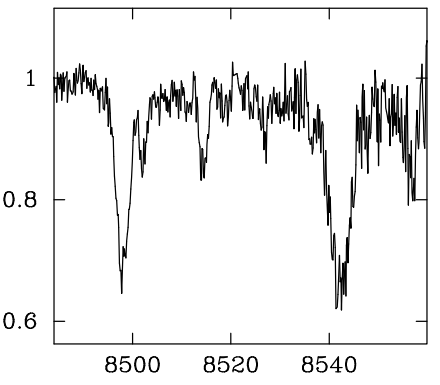

HD 37394

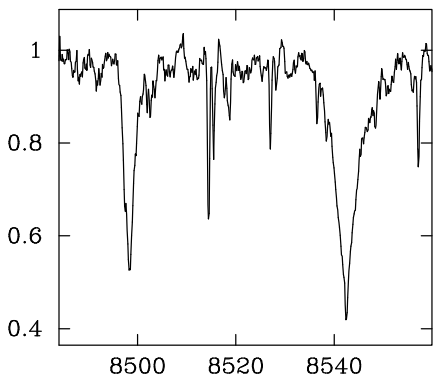

$\mathrm{BD}+201790$

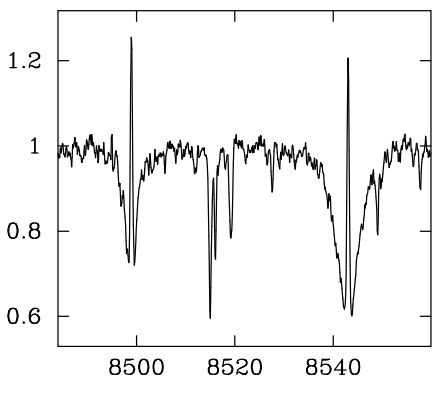

HD 72905

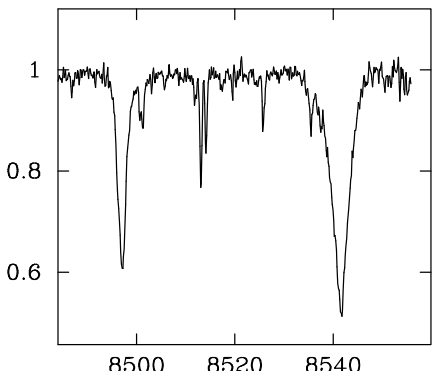



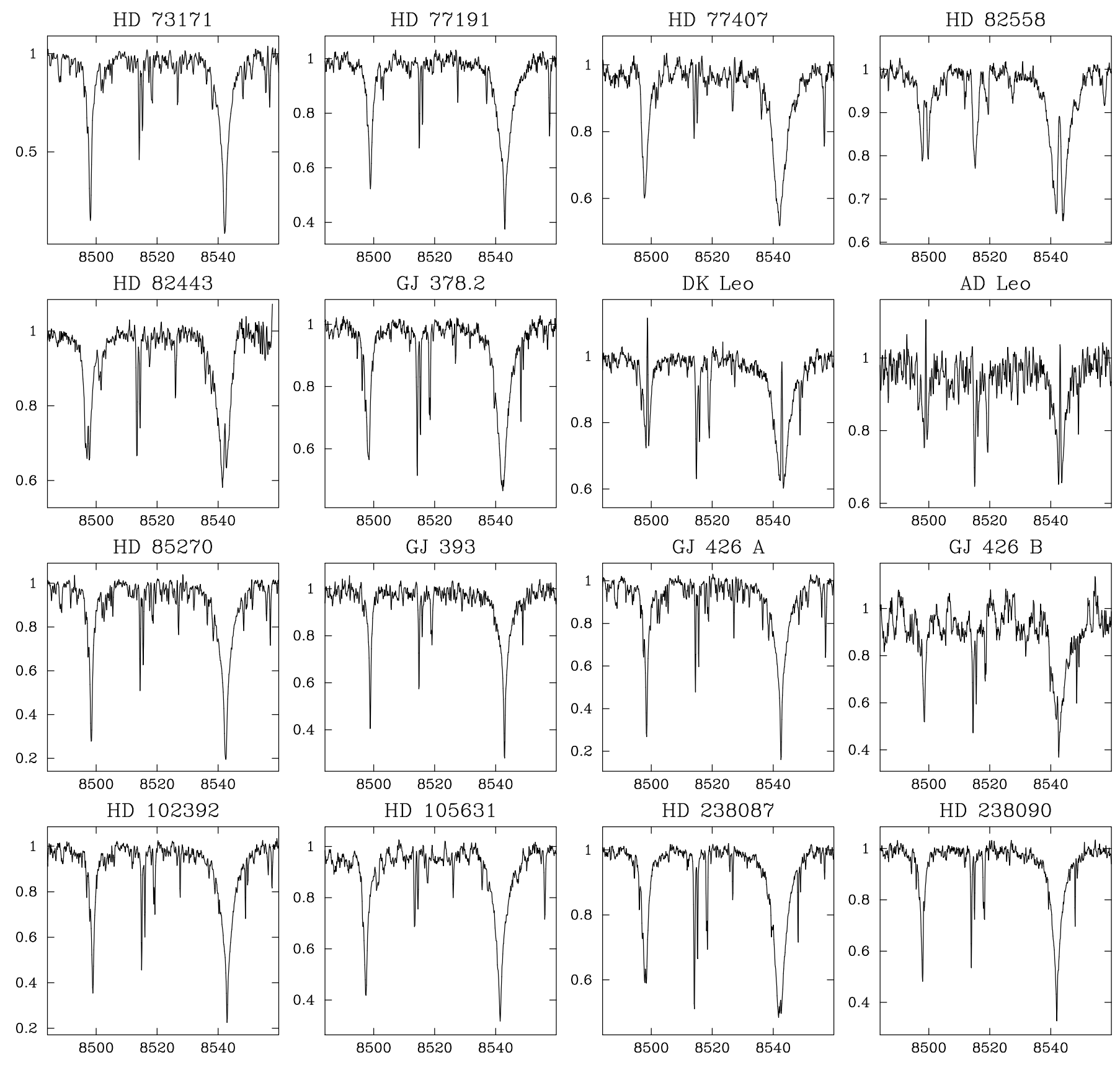

HD 238090
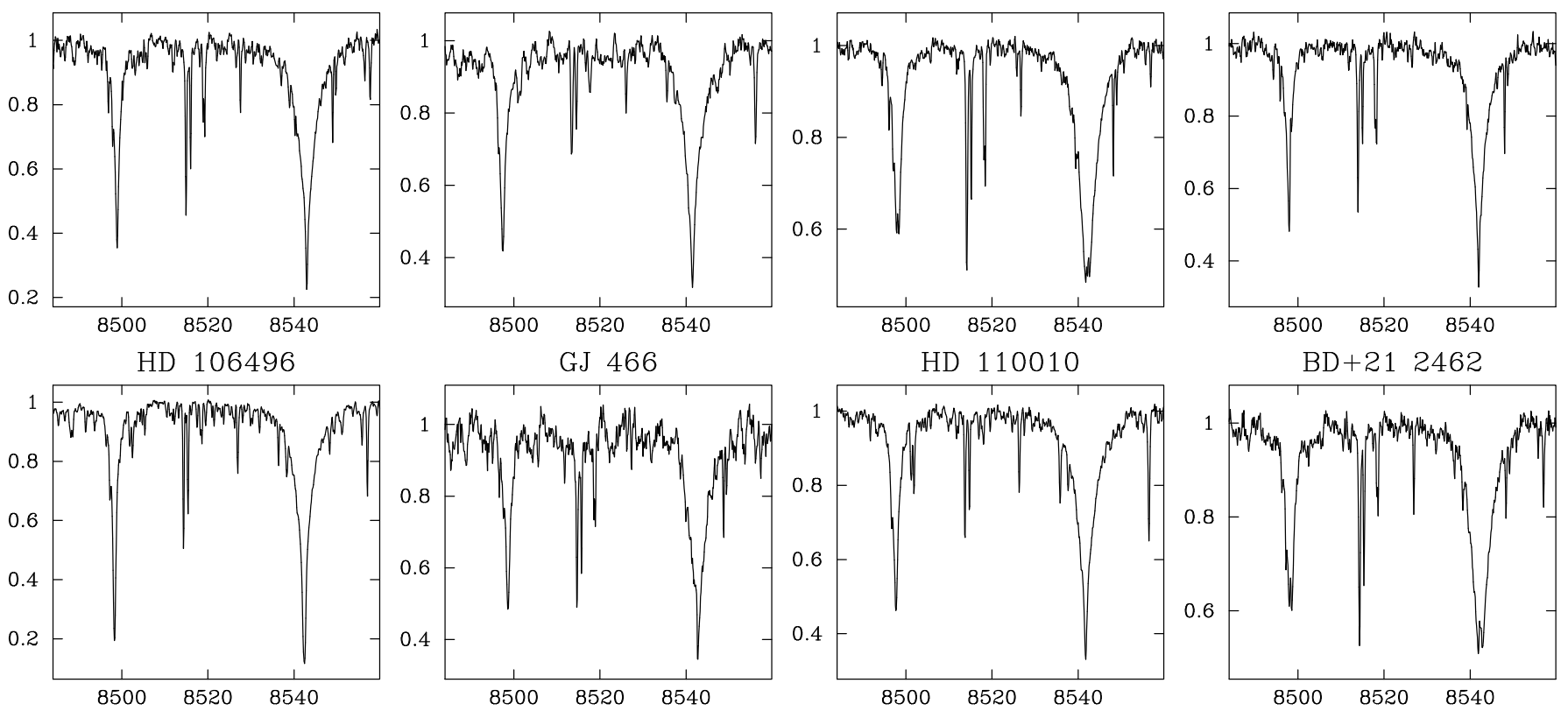

BD +21 2462

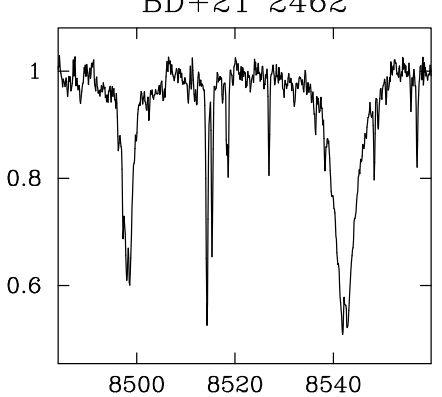

Fig. A.4. continued. 
J. López-Santiago et al.: High-resolution spectroscopic survey of late-type stars
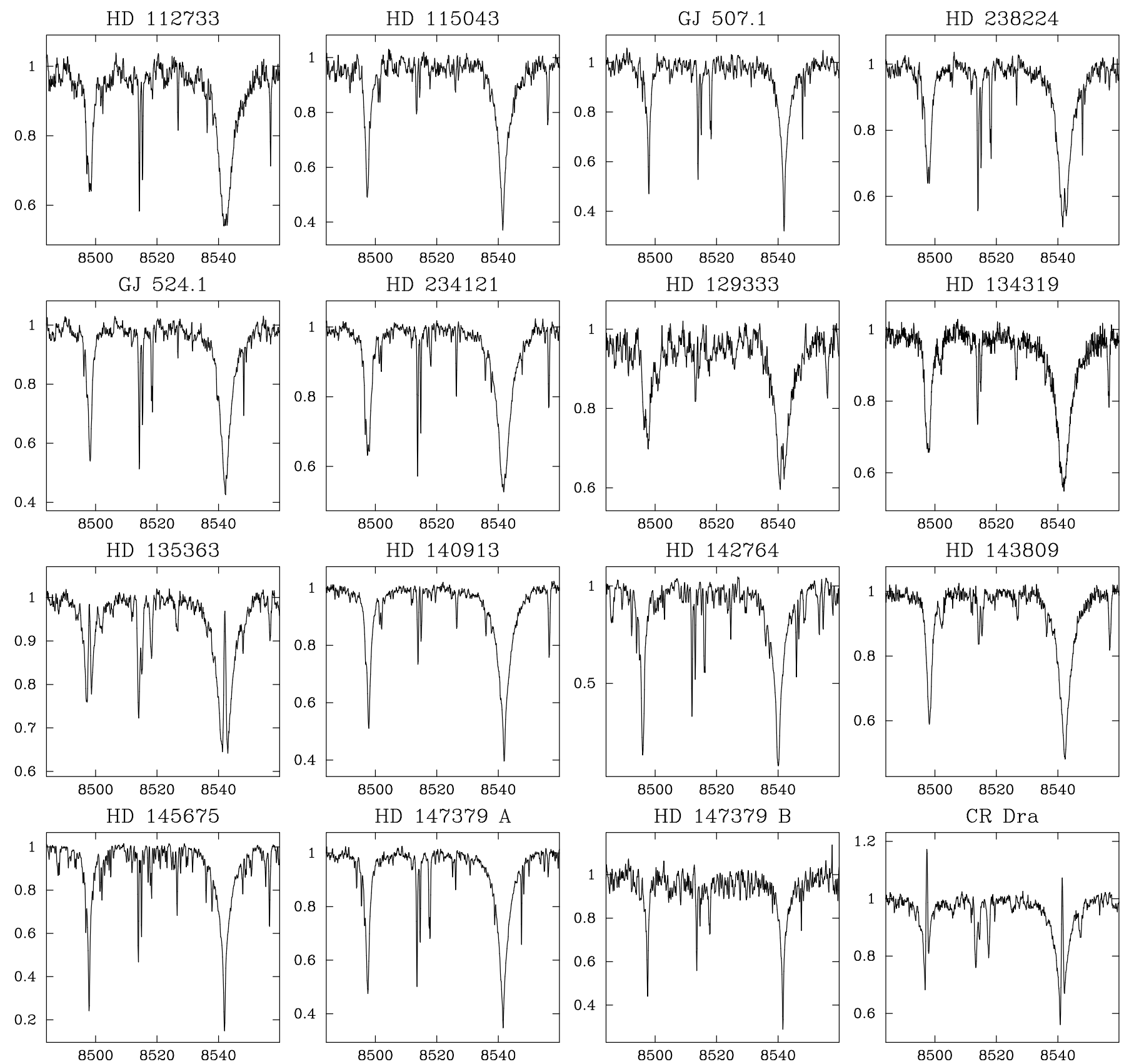

HD 147379 A
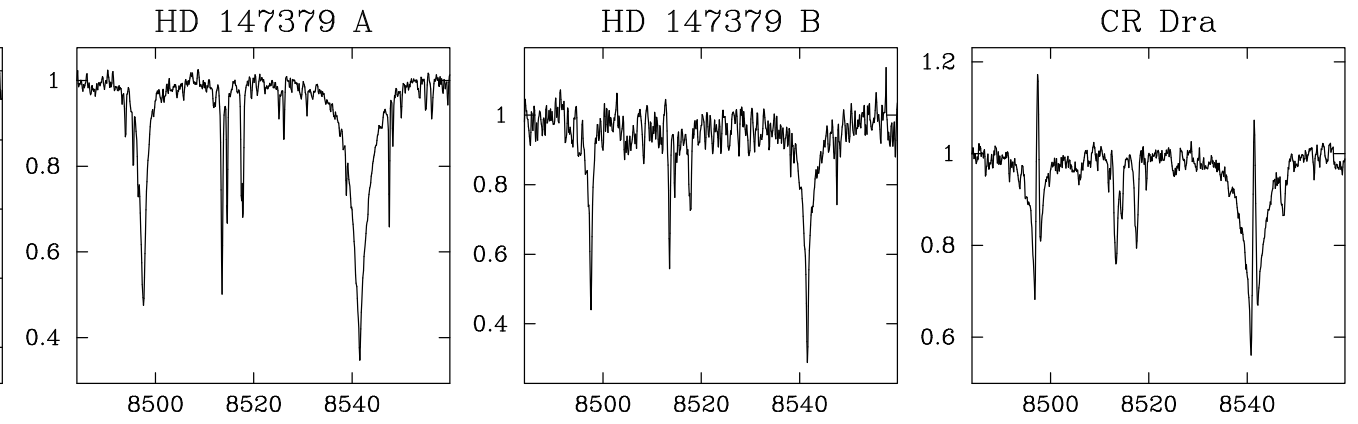

HD 149661

HD 152863
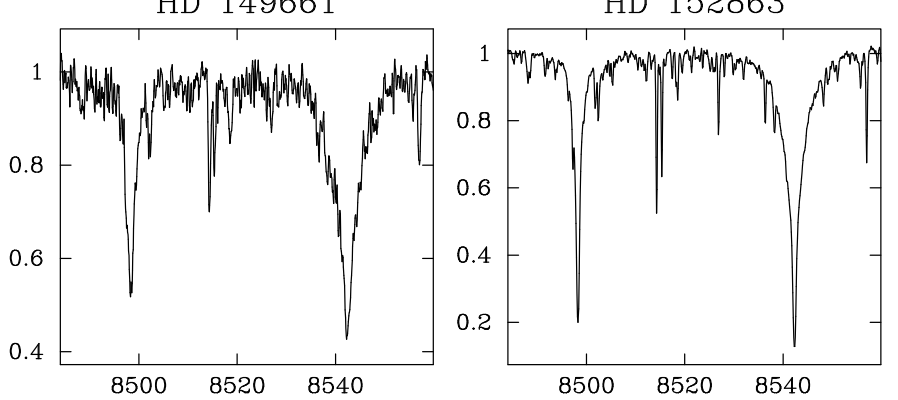

HD 152751

HD 155674 A
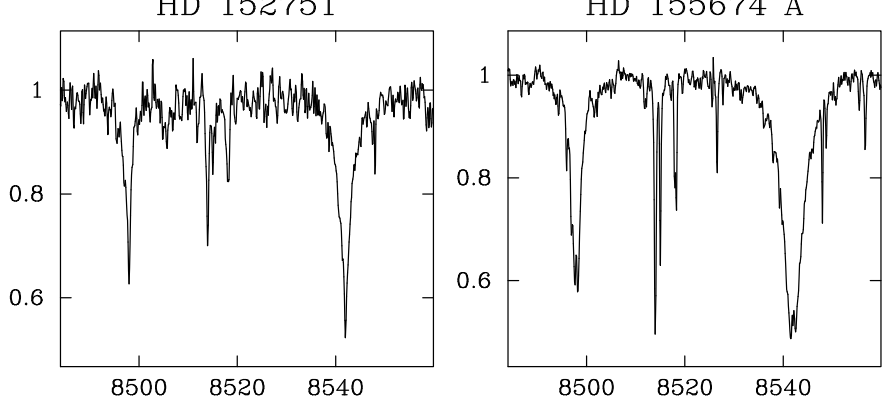

Fig. A.4. continued. 

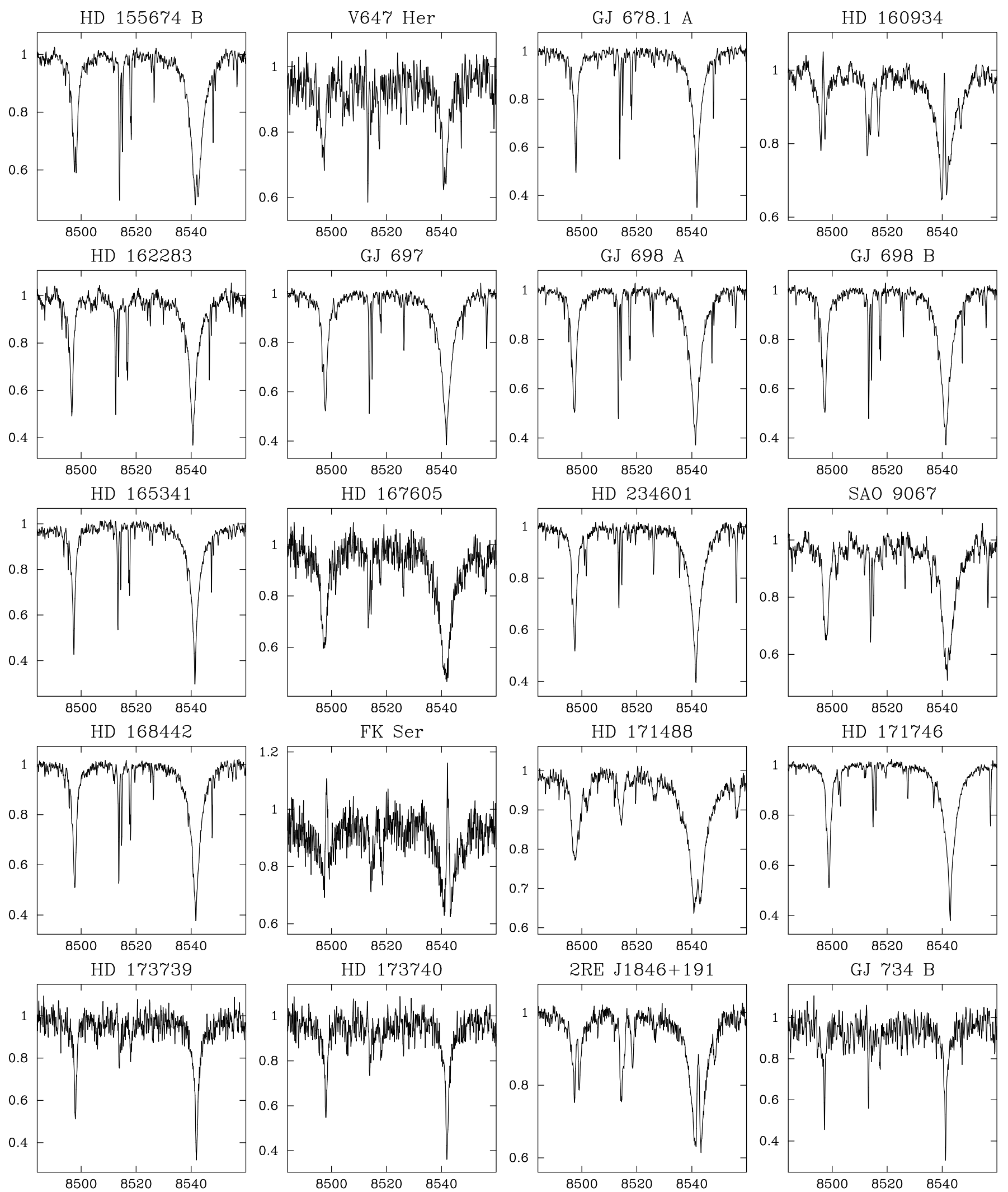

GJ 734 B

Fig. A.4. Continued. 
J. López-Santiago et al.: High-resolution spectroscopic survey of late-type stars
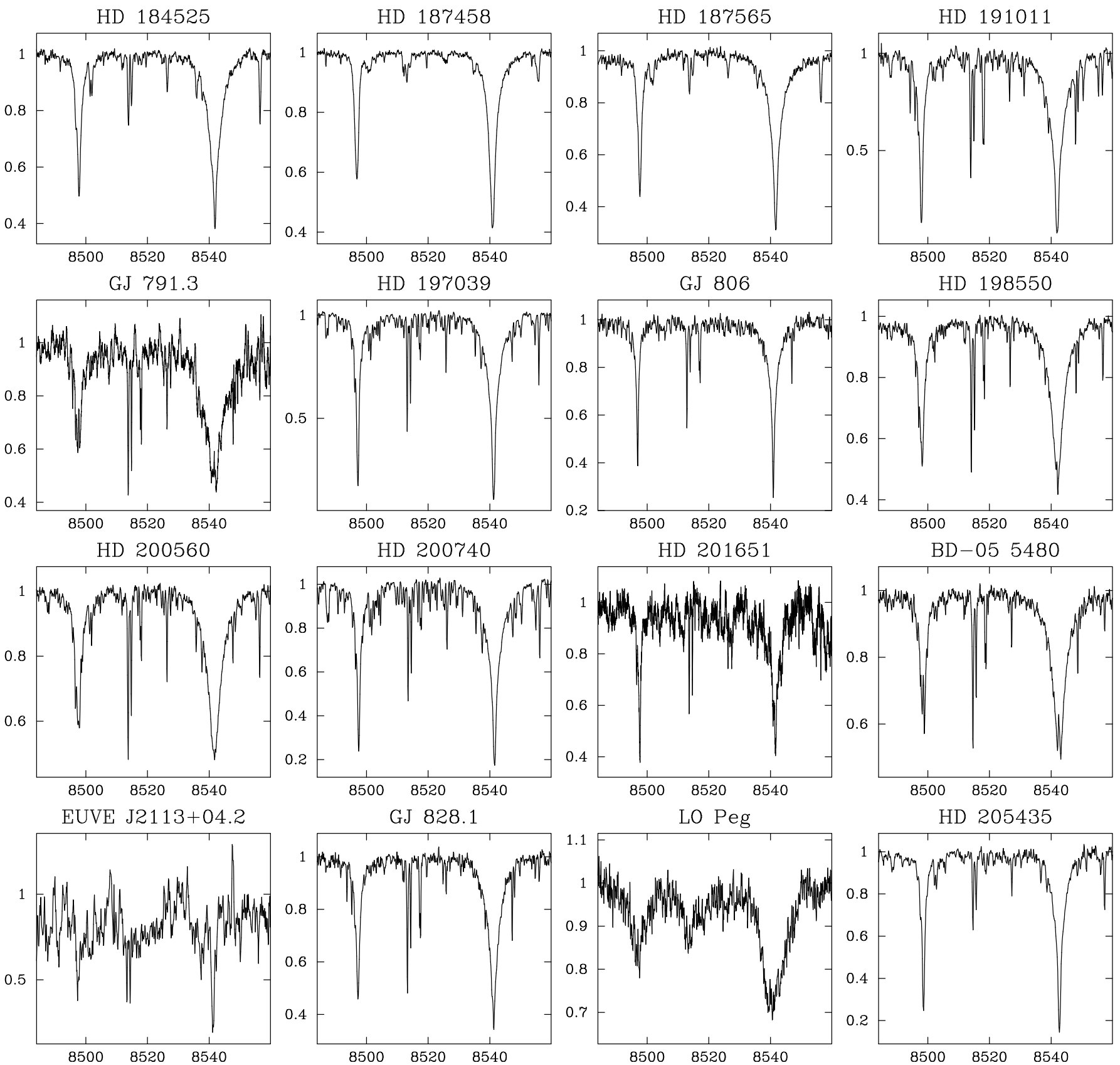

GJ 828.1

LO Peg

HD 205435
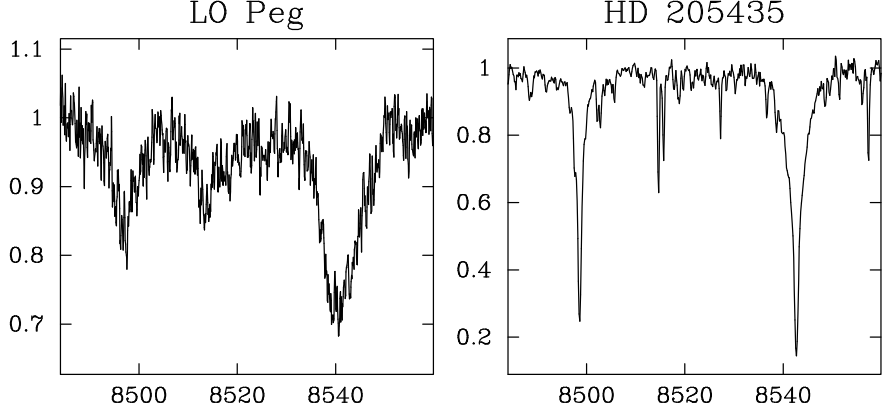

HD 206860

HD 208472

GJ 842.2
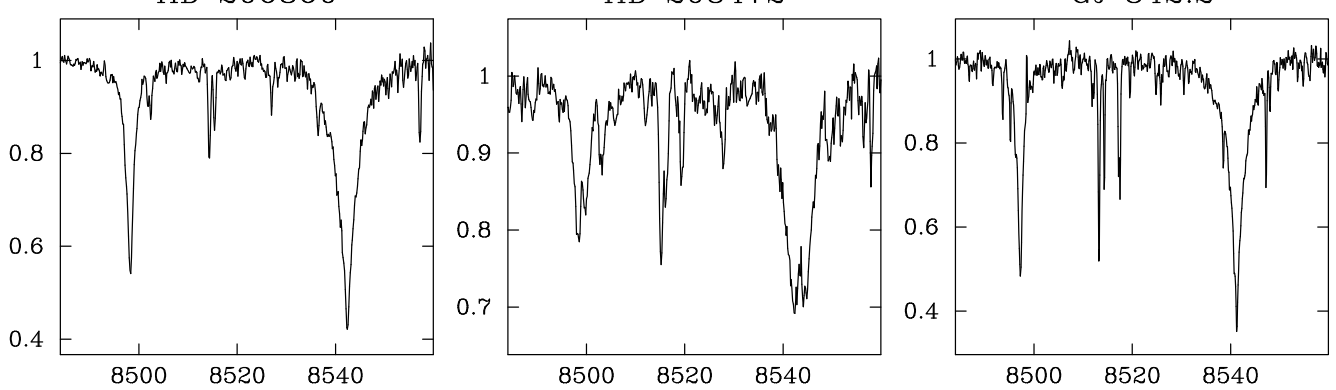

GJ 844

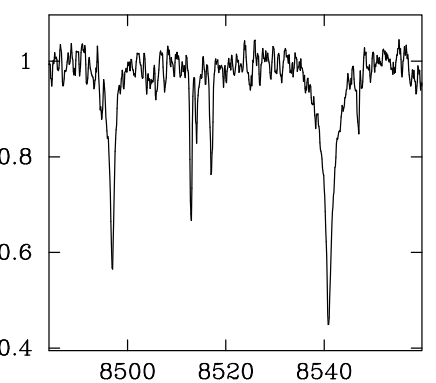

Fig. A.4. continued. 

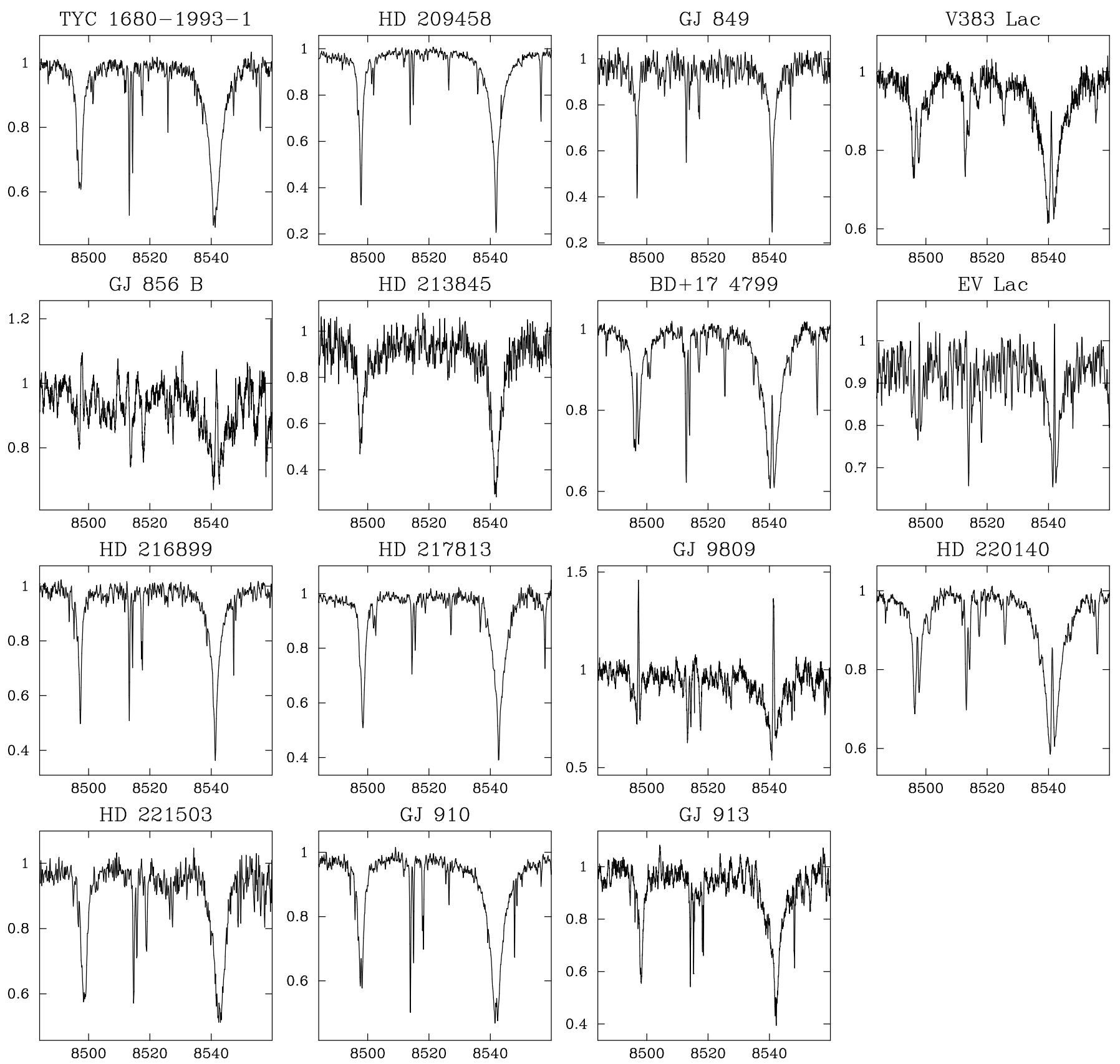

Fig. A.4. continued. 